\title{
MAGNETIC BOTTLES FOR THE NEUMANN PROBLEM: CURVATURE EFFECTS IN THE CASE OF DIMENSION 3 (GENERAL CASE)
}

\author{
By BERNARD HELFFER ${ }^{1}$ AND ABDEREMANE MORAME
}

\begin{abstract}
In a recent paper Lu and Pan have analyzed the asymptotic behavior, in the semi-classical regime, of the ground state energy of the Neumann realization of the Schrödinger operator in the case of dimension 3. Although these results are rather satisfactory when the magnetic field is non-constant and satisfies some generic conditions, they are not sufficient in the case of a constant magnetic field for understanding phenomena like the onset of superconductivity and more accurate results should be obtained. In the two-dimensional case, the effects due to the curvature of the boundary were predicted by a formal analysis of Bernoff-Sternberg and finally proved by the joint efforts of Lu-Pan, Del Pino-Felmer-Sternberg and Helffer-Morame. Our aim is to analyze similar effects in dimension 3. As known from physicists and roughly analyzed by $\mathrm{Lu}-\mathrm{Pan}$, it turns out that the results depend on the geometry of the boundary especially at the points where the magnetic field is tangent at the boundary. We present here the analog of the BernoffSternberg conjecture (also formulated in a different form by Pan) and prove it in the generic situation.
\end{abstract}

○ 2004 Published by Elsevier SAS

RÉSUMÉ. - Récemment Lu et Pan ont analysé le comportement asymptotique, en régime semi-classique, de la plus petite valeur propre de la réalisation de Neumann d'un opérateur de Schrödinger magnétique dans le cas de la dimension 3 . Si les résultats obtenus sont satisfaisants lorsque le champ magnétique est variable et vérifie quelques conditions génériques, ils ne permettent pas, dans le cas d'un champ magnétique constant, de comprendre des phénomènes comme la localisation d'une fonction propre associée. Dans le cas de la dimension 2, Bernoff et Sternberg avaient conjecturé, sur la base de constructions formelles, que c'était la courbure du bord qui allait être la clef de cette localisation. Ceci fut finalement prouvé par Lu-Pan, Del Pino-Sternberg et Helffer-Morame. Nous nous proposons ici d'analyser le même problème en dimension 3. La littérature physique indique (et les premiers résultats dans cette direction furent démontrés par Lu et Pan) que tout dépend de la géométrie de la frontière et plus précisément de la courbe (génériquement) où le champ magnétique est tangent au bord. Nous présentons ici l'analogue de la conjecture de BernoffSternberg dans le cas de la dimension 3 (conjecture aussi formulée sous une forme un peu différente par Pan) et en donnons la démonstration sous des hypothèses génériques.

○ 2004 Published by Elsevier SAS

\footnotetext{
${ }^{1}$ This work has been partially supported by the European Union under the TMR Program EBRFXT 960001 and the ESF program SPECT.
} 


\section{Introduction}

Our study is motivated by a question in superconductivity. ${ }^{2}$ Let us roughly explain how the question occurs in this context (see [10] or [22] for a review on this aspect and references therein). Given a bounded open set $\Omega \subset \mathbb{R}^{3}$ with smooth boundary, we are interested in the properties of the minimizers of the so called Ginzburg-Landau functional

$$
H^{1}(\Omega ; \mathbb{C}) \times H^{1}\left(\Omega ; \mathbb{R}^{3}\right) \ni(\psi, \widetilde{A}) \mapsto \mathcal{G}(\psi, \widetilde{A})
$$

with:

$$
\mathcal{G}(\psi, \widetilde{A})=\int_{\Omega}\left\{|(\nabla-i \kappa \widetilde{A}) \psi|^{2}+\frac{\kappa^{2}}{2}\left(|\psi|^{2}-1\right)^{2}\right\} d x+\kappa^{2} \int_{\Omega}|\operatorname{curl} \widetilde{A}-\sigma \operatorname{curl} A|^{2} d x
$$

Here $A$ is a given applied magnetic potential in $H^{1}\left(\Omega ; \mathbb{R}^{3}\right), \sigma$ is a positive parameter introduced for permitting a variation of its intensity and the parameter $\kappa>0$ reflects the properties of the sample $\Omega$ which will be assumed to be large. As $\Omega$ is bounded, the existence of a minimizer is rather standard. It is also easy to observe that the pair $(0, \sigma A)$ is a trivial critical point of the functional $\mathcal{G}$ which is called a normal solution. It has also be shown, that this pair is, when $\operatorname{curl} A:=H$ does not vanish, a global minimum for $\sigma$ large enough. It is therefore natural to discuss in function of $\sigma$, if this pair is a local or a global minimizer. By looking at the Hessian of the functional at this point, this question is immediately related to the positivity of the operator:

$$
-(\nabla-i \kappa \sigma A)^{2}-\kappa^{2} \quad \text { in } \Omega,
$$

where we get from the minimization of the functional a "magnetic" Neumann condition at the boundary.

It can be seen that the change of sign of the lowest eigenvalue of this operator occurs for $\sigma$ of the order of $\kappa$. To get a more precise information, we are immediately let to analyze the groundstate energy (that is the lowest eigenvalue) of the Neumann realization of the Schrödinger operator with magnetic potential in an open set $\Omega$ in $\mathbb{R}^{3}$ :

$$
P_{A}^{h}=\sum_{j=1}^{3}\left(h D_{x_{j}}-A_{j}(x)\right)^{2},
$$

where $h=\frac{1}{\kappa \sigma}$ will be a small parameter. This is this problem which will be the main object of the paper. We recall that an element $u$ in $H^{2}(\Omega)$ satisfies the (magnetic) Neumann condition at the boundary, if

$$
N(x) \cdot((h D-A) u)(x)=0, \quad \forall x \in \partial \Omega,
$$

where $N(x)$ is the normal at $x$ to $\partial \Omega$.

\footnotetext{
${ }^{2}$ One can find in Chapter 4 of [30] a physical presentation of the problem we are considering. We particularly emphasize on their Section 4.3 where they analyze (with partially heuristic arguments) the angular dependence of the nucleation field. We will give a mathematical proof of, what they describe for example p. 87: "For type II superconductors (The authors mean $\kappa \geqslant 1 / \sqrt{2}$, but we treat only $\kappa$ large) the above calculation shows that superconductivity is not entirely destroyed for $H_{c_{2}}<H<H_{c_{3}}$. A superconducting sheath remains close to the surface parallel to the applied field. Conversely, when the field is decreased below $H_{c_{3}}$, a superconducting sheath appears at the surface before superconductivity is restored in the bulk at $H=H_{c_{2}}$. If the sample is a long cylinder with the applied field parallel to the axis, the sheath will cover all the surface of the cylinder. If it is a sphere, the sheath will be restricted to a small zone near the equatorial plane when $H$ is close to $H_{c_{3}}$. When the field is decreased towards $H_{c_{2}}$ the sheath will progressively extend up to the poles".
} 
This realization will be denoted by $P_{A, \Omega}^{h, N}$ but we will sometimes use shorter notations when there is no ambiguity. If $\lambda(h)$ is the lowest eigenvalue and $u^{h}(x)$ is a corresponding normalized eigenstate, we would like to discuss the asymptotic of $\lambda(h)$ as $h \rightarrow 0$, with the hope to analyze in a future work the localization of $u^{h}$. This problem was treated in the case of dimension 2 by Bernoff-Sternberg [3], Lu-Pan [19-21], Del Pino-Felmer-Sternberg [28] and Helffer-Morame [12] in a rather complete way. Later a recent work by Lu-Pan [23] was starting the analysis of the dimension 3 and this was complemented slightly in [13]. So this paper is the continuation of the program, with a particular emphasis on the constant magnetic field case.

We start by establishing rough estimates, but sufficiently accurate for determining the effective order of the curvature effect.

THEOREM 1.1. - Let $\Omega$ be a bounded open set of $\mathbb{R}^{3}$ with $C^{\infty}$ boundary $\partial \Omega$. Let $P_{A, \Omega}^{h, N}$ be the Neumann operator on $L^{2}(\Omega)$ associated to the Schrödinger operator with constant magnetic field $(h D-A)^{2}$ and let us assume that the vector magnetic field $H=\operatorname{curl}(A)$ is constant and of intensity equal to $b=|H|$.

If

$$
\lambda(h)=\inf \operatorname{Sp}\left(P_{A, \Omega}^{h, N}\right)
$$

is the first eigenvalue of $P_{A, \Omega}^{h, N}$, then there exists a constant $C_{0}$ such that

$$
\left|\lambda(h)-b \Theta_{0} h\right| \leqslant C_{0} h^{4 / 3}
$$

where $\left.\Theta_{0} \in\right] 0,1[$ is an universal constant, which will be defined in (2.4).

We observe that in the case of dimension 2, the corresponding error was in $\mathcal{O}\left(h^{3 / 2}\right)$.

We then continue with the analysis of the curvature effects, which are the analog in dimension 3 of the results first conjectured by Bernoff-Sternberg (see [3]) in the case of dimension 2. Before establishing the corresponding conjecture in the case of dimension 3 , let us describe the main ingredients appearing in the assumptions.

It has been observed by [23] and this will be recalled in Section 4, that the ground state $u^{h}$ is localized (as $h \rightarrow 0$ ) near the boundary $\partial \Omega$ but more precisely on the set:

$$
\Gamma_{H}=\{x \in \partial \Omega \mid\langle H \mid N(x)\rangle=0\},
$$

that is the set of points in $\partial \Omega$ where $H$ is tangent.

It is natural to assume that:

$\Gamma_{H}$ is a regular submanifold of $\partial \Omega$,

that is a disjoint union of regular curves. From now on, we choose an orientation on each curve. At each point $x$ of $\Gamma_{H}$, we will associate the normal curvature along the magnetic field $H=H(B)$ by:

$$
\kappa_{n, B}(x):=K_{x}\left(T(x) \wedge N(x), \frac{H}{|H|}\right),
$$

where $K$ denotes the second fundamental form on the surface $\partial \Omega$ (see Section 8 ) and $T(x)$ is the unit oriented tangent vector to $\Gamma_{H}$ at $x$. It is natural to assume that:

$$
\kappa_{n, B} \neq 0, \quad \text { on } \Gamma_{H} .
$$


The last generic assumption is:

The set of points where $H$ is tangent to $\Gamma_{H}$ is isolated.

The following function on $\Gamma_{H}$ will play an important role:

$$
\tilde{\gamma}_{0}(x)=\left(\frac{1}{2}\right)^{2 / 3} \hat{\nu}_{0} \delta_{0}^{1 / 3}\left(\left|\kappa_{n, B}(x)\right|^{2 / 3}\left(\delta_{0}+\left(1-\delta_{0}\right)\left|\left\langle T(x) \mid \frac{H}{|H|}\right\rangle\right|^{2}\right)^{1 / 3}\right),
$$

where $\hat{\nu}_{0}>0$ and $\left.\delta_{0} \in\right] 0,1[$ are universal constants attached to spectral invariants related to two model Hamiltonians respectively defined on $\mathbb{R}$ and $\mathbb{R}^{+}$and which will be defined in (2.15) and (2.7).

Associated to this function, which will play the role of effective curvature, we define:

$$
\hat{\gamma}_{0}=\inf _{x \in \Gamma_{H}} \tilde{\gamma}_{0}(x)
$$

Our main theorem is:

THEOREM 1.2. - Let $P_{A, \Omega}^{h, N}$ be the Neumann realization on $L^{2}(\Omega)$ of the magnetic Laplacian $(h D-A)^{2}$, where $\left.h \in\right] 0,1[$ is a small parameter and A corresponds to constant magnetic field. Under assumptions (1.5), (1.7) and (1.8), there exist $\eta>0$ and $\hat{\gamma}_{0}>0$ such that:

$$
\inf \operatorname{Sp}\left(P_{A, \Omega}^{h, N}\right)=b \Theta_{0} h+\hat{\gamma}_{0} b^{2 / 3} h^{4 / 3}+\mathcal{O}\left(h^{\frac{4}{3}+\eta}\right),
$$

where $\hat{\gamma}_{0}$ is defined in (1.10).

We conjectured this theorem in September 2001, simultaneously with Pan [26] (who proposed another equivalent ${ }^{3}$ formulation and obtained the upper bound). A first complete proof was given in mp_arc [14] under additional non generic conditions. This paper, which is a slightly modified version of the preprint [15], gives now a complete proof in the general generic case. Although the methods of proof can also lead to localization results for the ground state (see $[12,13]$ ) or more generally for minimizers of the Ginzburg-Landau functional (see [19-23,17]), this will not be discussed here. This was actually explored in [26], under the assumption that the conjecture was true.

The paper is organized as follows.

In Sections 2 and 3, we recall previous results extracted from [6,12,23,13], which will play an important role in the analysis. In Section 4, we recall the results of [23] devoted to the case when the magnetic field is not constant. It is also shown there that the problem is reduced in the constant magnetic case to a neighborhood of the boundary. In Section 5, we make explicit our choice of coordinates near the boundary. Section 6 gives rough upper bounds for the ground state energy by constructing quasimodes. In Section 7, we present our first lower bounds which are sufficiently accurate for giving the right order for the remainder. In Section 8, we go further in the choice of adapted coordinates, taking in particular account of the fact that the magnetic field is constant. This permits to introduce our magnetic invariants attached to $\Gamma_{H}$ and to present our main results in a more precise form. Section 9 is devoted to the research of simpler models for the magnetic potentials obtained in the adapted coordinates by suitable gauge transformations and by neglecting "small" terms. Section 10 is devoted to the estimate of the errors done in the

\footnotetext{
${ }^{3}$ See the appendix in [15].
} 
use of the previous models. In Section 11, we start by some heuristics which lead to the spectral analysis of a simplified model which will be used in the general case. Section 12 is devoted to the proof of the accurate upper bounds. Section 13 gives complementary lower bounds for the model. Section 14 presents the structure of the proof of accurate lower bounds according to different zones and treats the easy zones. Sections 15 and 16 correspond to the treatment of the difficult zones.

\section{The case of dimension 2}

\subsection{Main results for the models with constant magnetic fields}

We first consider the operator:

$$
P_{A}^{h}:=\left(h D_{x_{1}}-A_{1}\right)^{2}+\left(h D_{x_{2}}-A_{2}\right)^{2},
$$

with

$$
A=\left(-\frac{b}{2} x_{2}, \frac{b}{2} x_{1}\right), \quad h>0 \quad \text { and } \quad b>0,
$$

and analyze the spectrum of its realization in $\mathbb{R}^{2}$ or of its Neumann realization in $\mathbb{R}_{+}^{2}$.

We observe that by homogeneity, one can reduce the analysis to $h=1$ and $b=1$. It is well known that in the case of $\mathbb{R}^{2}$ the spectrum is a point spectrum and that the eigenvalues are given by $(2 n+1)$ with $(n \in \mathbb{N})$, each eigenvalue being of infinite multiplicity. One way, to see this is to show the unitary equivalence (via a gauge transformation, a partial Fourier transform and a translation) with the harmonic oscillator $\left(D_{t}^{2}+t^{2}\right)$ but seen as an unbounded operator on $L^{2}\left(\mathbb{R}_{t, s}^{2}\right)$.

The case of the Neumann realization in $\mathbb{R}_{+}^{2}$ is a little more delicate. By unitary transformation we get the Neumann realization of the operator of

$$
Q\left(t, D_{t} ; s\right):=D_{t}^{2}+(t-s)^{2} \quad \text { on } L^{2}\left(\mathbb{R}_{+} \times \mathbb{R}\right)
$$

which is reduced to the analysis of the family $(s \in \mathbb{R})$ of operators:

$$
Q(s):=D_{t}^{2}+(t-s)^{2},
$$

defined on $L^{2}\left(\mathbb{R}_{+}\right)$.

Let $\mu(s)$ be defined by:

$$
\mu(s) \text { is the lowest eigenvalue of the Neumann realization of } Q(s) \text { in } \mathbb{R}^{+} .
$$

One easily shows (cf. [29])

PROPOSITION 2.1. - The infimum of the spectrum of the Neumann realization of $P_{A}^{h}$ is given by bh $\Theta_{0}$ with

$$
\Theta_{0}:=\inf _{s \in \mathbb{R}} \mu(s)
$$




\subsection{An important one-dimensional family of operators on $\mathbb{R}$}

We recall the main properties of the groundstate energy of the Neumann realization on $\mathbb{R}^{+}$of

$$
Q(s):=D_{t}^{2}+(t-s)^{2},
$$

in function of the parameter $s \in \mathbb{R}$. Let us just list some properties of $\mu(s)$ and of the corresponding normalized eigenvector $\varphi^{s}$ and refer to [6,12] for proofs or details. The Neumann eigenvalue $\mu(s)$ satisfies the following properties:

1. $\lim _{s \rightarrow-\infty} \mu(s)=+\infty$;

2. $\mu$ is decreasing for $s<0$;

3. $\mu(0)=1$;

4. $\lim _{s \rightarrow+\infty} \mu(s)=1$;

5. $\mu$ admits in $] 0,+\infty\left[\right.$ a unique minimum $\Theta_{0}<1$ at some $\xi_{0}>0$. So

$$
\Theta_{0}:=\inf _{s \in \mathbb{R}} \mu(s)=\mu\left(\xi_{0}\right)<1 .
$$

This minimum is nondegenerate and more precisely:

$$
0<\delta_{0}:=\frac{1}{2} \mu^{\prime \prime}\left(\xi_{0}\right)<1
$$

6. We introduce $\varphi_{0}=\varphi^{\xi_{0}}$. We have:

$$
\int_{\mathbb{R}_{+}}\left(t-\xi_{0}\right)\left|\varphi_{0}(t)\right|^{2} d t=0,
$$

which just corresponds to the condition $\mu^{\prime}\left(\xi_{0}\right)=0$.

7. $\varphi_{0}$ is rapidly decreasing at $\infty$.

Let us just mention that the proof of some of these properties is based on the following identity, relating the first eigenfunction $\varphi^{s}$ and $\mu(s)$ :

$$
\left\|\varphi^{s}\right\|^{2} \mu^{\prime}(s)=\left(s^{2}-\mu(s)\right)\left(\varphi^{s}(0)\right)^{2} .
$$

\subsection{Applications: main results in dimension 2}

As an application of the analysis of the models, one gets (cf. [21]) via a partition of unity for the lower bound and a suitable construction of quasimodes for the upper bound, the following general result (in the two-dimensional case):

THEOREM 2.2. - If $\lambda(h)$ is the lowest eigenvalue of the Neumann realization of $P_{A}^{h}$ in $\Omega$, then we have:

$$
\lim _{h \rightarrow 0} \frac{\lambda(h)}{h}=\min \left(\inf _{x \in \Omega}|B(x)|, \Theta_{0} \inf _{x \in \partial \Omega}|B(x)|\right)
$$

where $B=\operatorname{curl} A$.

We emphasize that there is no assumption that the magnetic field is constant. In the case of the constant magnetic field, one can actually have more precise results for $\lambda(h)$. When $B$ is constant, the minimum in (2.10) is obtained by the second term and we showed in $[3,28,12]$ the 
THEOREM 2.3. - If the magnetic field is constant of intensity $b$, then:

$$
\lambda(h)=b \Theta_{0} h-c_{1} b^{1 / 2}\left(\sup _{x \in \partial \Omega} \kappa(x)\right) h^{3 / 2}+\mathrm{o}\left(h^{3 / 2}\right),
$$

where $c_{1}$ is a universal strictly positive universal constant and $\kappa(x)$ is the curvature of $\partial \Omega$ at $x \in \partial \Omega$.

\subsection{The Montgomery's model and a second important family of operators on $\mathbb{R}$}

We just discuss a model that we shall meet indirectly later and which is interesting. It first appears in [24] but see also [11]. We consider in $\mathbb{R}_{x, y}^{2}$, and for some parameter $\kappa>0$ the operator:

$$
P:=h^{2} D_{x}^{2}+\left(h D_{y}-\frac{\kappa}{2} x^{2}\right)^{2} .
$$

The magnetic potential is $\vec{A}=\left(0, \frac{\kappa}{2} x^{2}\right)$ and we have:

$$
\operatorname{curl} A=\kappa x .
$$

So the magnetic field vanishes along the line $\{x=0\}$. Let us briefly describe the spectral analysis. After a Fourier transform in the $y$-variable, we first get:

$$
\hat{P}=h^{2} D_{x}^{2}+\left(h \eta-\frac{\kappa}{2} x^{2}\right)^{2}
$$

and this leads to the analysis of the family, parametrized by $\eta \in \mathbb{R}$, of selfadjoint operators on $L^{2}(\mathbb{R})$ :

$$
\hat{P}(\eta)=h^{2} D_{x}^{2}+\left(h \eta-\frac{\kappa}{2} x^{2}\right)^{2} .
$$

Using a simple dilation, we get:

$$
\inf \operatorname{Sp}(\hat{P})=\inf _{\eta} \inf \operatorname{Sp}(\hat{P}(\eta))=h^{4 / 3}\left|\frac{\kappa}{2}\right|^{2 / 3} \inf _{\rho} \inf \operatorname{Sp}\left(D_{r}^{2}+\left(r^{2}-\rho\right)^{2}\right) .
$$

Let us recall some properties of the family of operators

$$
S(\rho)=D_{r}^{2}+\left(r^{2}-\rho\right)^{2},
$$

and of the corresponding ground state $\psi^{\rho}$, which were established in [24,11] and [27].

1. There exists a unique $\rho=\rho_{\min }$ such that:

$$
\hat{\nu}_{0}:=\inf _{\rho} \inf \operatorname{Sp}\left(D_{r}^{2}+\left(r^{2}-\rho\right)^{2}\right)=\inf \operatorname{Sp}\left(D_{r}^{2}+\left(r^{2}-\rho_{\min }\right)^{2}\right) .
$$

2. $\psi^{\rho}$ belongs to $\mathcal{S}(\mathbb{R})$ and is even.

We shall later use the notation:

$$
\psi_{0}=\psi^{\rho_{\min }}
$$




\section{Constant magnetic field: models in $\mathbb{R}^{3}$ and in $\mathbb{R}_{+}^{3}$}

As in the case of dimension 2 , where the first thing was to understand the model with constant magnetic field in $\mathbb{R}^{2}$ and $\mathbb{R}_{+}^{2}$, we shall discuss the case of $\mathbb{R}^{3}$ and $\mathbb{R}_{+}^{3}$.

\subsection{Model in $\mathbb{R}^{3}$}

We start (after some gauge transformation) of the Schrödinger operator with constant magnetic field in dimension 3.

$$
P^{h}(\vec{H}):=h^{2} D_{x_{1}}^{2}+\left(h D_{x_{2}}-H_{3} x_{1}\right)^{2}+\left(h D_{x_{3}}+H_{2} x_{1}-H_{1} x_{2}\right)^{2} .
$$

The following lemma has the age of quantum mechanics:

LEMMA 3.1. - The bottom of the spectrum of the selfadjoint realization of $P^{h}(\vec{H})$ in $\mathbb{R}^{3}$ is

$$
\inf \operatorname{Sp}\left(P_{\mathbb{R}^{3}}^{h, N}(\vec{H})\right)=b h,
$$

where

$$
b=|H|=\sqrt{H_{1}^{2}+H_{2}^{2}+H_{3}^{2}}
$$

is the intensity of $H$.

\subsection{Models in halfspaces}

We refer to [23] and [13] for the proof of the results presented in this subsection. If $N$ is a unit vector in $\mathbb{R}^{3}$, we now consider the Neumann realization in $\Omega:=\left\{x \in \mathbb{R}^{3} \mid x \cdot N>0\right\}$. After a rotation, we can assume in the proofs that $N=(1,0,0)$, so $\Omega$ is $\mathbb{R}_{+}^{3}:=\left\{x_{1}>0\right\}$.

After scaling, we can assume that $h=1$ and $|H|=1$.

After some rotation in the $\left(x_{2}, x_{3}\right)$ variables, we can assume that the new magnetic field is $\left(\beta_{1}, \beta_{2}, 0\right)$ and we are reduced to the problem of analyzing:

$$
P\left(\beta_{1}, \beta_{2}\right):=D_{x_{1}}^{2}+D_{x_{2}}^{2}+\left(D_{x_{3}}+\beta_{2} x_{1}-\beta_{1} x_{2}\right)^{2},
$$

in $\left\{x_{1}>0\right\}$, where:

$$
\beta_{1}^{2}+\beta_{2}^{2}=1
$$

We introduce:

$$
\beta_{2}=\cos \vartheta, \quad \beta_{1}=\sin \vartheta,
$$

and we observe that, if $N$ is the external normal to $x_{1}=0$, we have:

$$
\langle\vec{H} \mid N\rangle=-\sin \vartheta
$$

By partial Fourier transform, we arrive to:

$$
L(\vartheta, \tau)=D_{x_{1}}^{2}+D_{x_{2}}^{2}+\left(\tau+\cos \vartheta x_{1}-\sin \vartheta x_{2}\right)^{2},
$$

in $x_{1}>0$ and with Neumann condition on $x_{1}=0$. The bottom of the spectrum of $L(\vartheta, \tau)$ is given by:

$$
\sigma(\vartheta):=\inf \operatorname{Sp}\left(L\left(\vartheta, D_{t}\right)\right)=\inf _{\tau}(\inf \operatorname{Sp}(L(\vartheta, \tau)))
$$


PROPOSITION 3.2. - The bottom of the spectrum of the Neumann realization of $P^{h}(\vec{H})$ in $\Omega:=\left\{x \in \mathbb{R}^{3} \mid x \cdot N>0\right\}$ is:

$$
\inf \operatorname{Sp} P_{\Omega}^{h, N}(\vec{H})=\sigma(\vartheta) b h,
$$

where $\vartheta \in\left[-\frac{\pi}{2}, \frac{\pi}{2}\right]$ is defined by (3.3).

By symmetry considerations, we observe also that:

$$
\sigma(\vartheta)=\sigma(-\vartheta)=\sigma(\pi-\vartheta) .
$$

It is consequently enough to look at the restriction to $\left[0, \frac{\pi}{2}\right]$.

\subsection{Properties of $\vartheta \mapsto \sigma(\vartheta)$}

Let us now list the main properties of the function $\vartheta \mapsto \sigma(\vartheta)$ on $\left[0, \frac{\pi}{2}\right]$. Most of them are established in [23] but see also [13].

1. $\sigma$ is continuous on $\left[0, \frac{\pi}{2}\right]$.

2 .

$$
\sigma(0)=\Theta_{0}<1
$$

3.

$$
\sigma\left(\frac{\pi}{2}\right)=1
$$

4.

$$
\sigma(\vartheta) \geqslant \Theta_{0}(\cos \vartheta)^{2}+(\sin \vartheta)^{2}
$$

5. If $\vartheta \in] 0, \frac{\pi}{2}[$, the spectrum of $L(\vartheta, \tau)$ is independent of $\tau$ and its essential spectrum is contained in $[1,+\infty[$.

6. For $\vartheta \in] 0, \frac{\pi}{2}[, \sigma(\vartheta)$ is an isolated eigenvalue of $L(\vartheta, \tau)$, with multiplicity one.

7. The function $\sigma$ is strictly increasing on $\left[0, \frac{\pi}{2}[\right.$.

8. The function $\sigma$ has the following expansion for $\vartheta$ small:

$$
\sigma(\vartheta) \sim \Theta_{0}+\sum_{n \geqslant 1} \alpha_{n}|\vartheta|^{n}
$$

with $\alpha_{1}=\sqrt{\delta}_{0}=\sqrt{\mu^{\prime \prime}\left(\xi_{0}\right) / 2}>0$.

A first consequence of this analysis is

Proposition 3.3. - When $b=|H|$ is fixed the bottom of the spectrum of $P_{\Omega}^{h, N}(\vec{H})$ in $\Omega:=\{x \cdot N>0\}$ is minimal when $\vartheta=0$ that is, according to (3.3), that is when the magnetic field vector satisfies $\vec{H} \cdot N=0$.

\section{First results for general magnetic fields}

In the case of dimension 2 , under the assumption that $\operatorname{curl} A:=B(x)>0$, the basic estimate at the interior was the inequality:

$$
h \int B(x)|u(x)|^{2} d x \leqslant \int|(h \nabla-i A) u|^{2} d x, \quad \forall u \in C_{0}^{\infty}(\Omega) .
$$


This was not enough for understanding the Neumann problem. One should more carefully analyze the case of $\mathbb{R}_{+}^{2}$ and, in the case of the constant magnetic field, one should also analyze more complicate models (like for example the case of the disk). The most spectacular result was:

THEOREM 4.1.- When the magnetic field is constant, the groundstate of the Neumann realization of $P_{A}^{h}$ in an open regular bounded set $\Omega \subset \mathbb{R}^{2}$ is localized in the neighborhood of the points of the boundary of maximal curvature.

In the case of dimension 3, estimate (4.1) should be replaced by the weaker estimate established in [11] (Theorem 3.1)

THEOREM 4.2. - There exist $C$ and $h_{0}>0$ such that, for all $\left.\left.h \in\right] 0, h_{0}\right]$, we have:

$$
h \int_{\Omega}\left(|H(x)|-C h^{1 / 4}\right)|u(x)|^{2} d x \leqslant \int_{\Omega}|(h \nabla-i A) u|^{2} d x, \quad \forall u \in C_{0}^{\infty}(\Omega) .
$$

If this result is essentially sufficient for analyzing the Dirichlet problem in $\Omega$, it is necessary to implement the analysis given in the first part in order to treat the Neumann problem. Near each point of the boundary $x$, we have to use the lower bound obtained for the model with constant magnetic field $H=H(x)$. Following for example the proof in [12] and using the same partition of unity, we get:

\section{THEOREM 4.3.-}

$$
h \int_{\Omega} W_{h}(x)|u(x)|^{2} d x \leqslant \int|(h \nabla-i A) u|^{2} d x, \quad \forall u \in H^{1}(\Omega),
$$

where

$$
W_{h}(x)= \begin{cases}|H(x)|-C h^{1 / 4}, & \text { if } d(x, \partial \Omega) \geqslant 2 h^{3 / 8}, \\ |H(s(x))| \sigma(\vartheta(x))-C h^{1 / 4} & \text { if } d(x, \partial \Omega) \leqslant 2 h^{3 / 8} .\end{cases}
$$

Here we recall that $\vartheta(x)$ satisfies:

$$
|H(s(x))| \cdot \sin \vartheta(x)=-\langle H(s(x)) \mid N(s(x))\rangle,
$$

where $s(x)$ is, for $x$ near $\partial \Omega$, the point in $\partial \Omega$ such that:

$$
d(x, \partial \Omega)=d(x, s(x))
$$

and we observe that, due to (3.7), $\sigma(\vartheta(x))$ is well defined by (4.5).

The first consequence (compare with Theorem 2.2) is:

THEOREM 4.4.-

$$
\lim _{h \rightarrow 0}(\lambda(h) / h)=\min \left(\inf _{x \in \Omega}|H(x)|, \inf _{x \in \partial \Omega}|H(x)| \sigma(\vartheta(x))\right) .
$$

The lower bound is a direct consequence of Theorem 4.3 and the proof of the upper bound is sketched in [23]. When $H(x)$ is constant of intensity $b$, then the minimum in (4.6) is obtained for the second term and we have:

$$
\lim _{h \rightarrow 0}(\lambda(h) / h)=b\left(\inf _{x \in \partial \Omega} \sigma(\vartheta(x))\right) .
$$


In view of (6.3), there exists a point $x$ such that $\vartheta(x)=0$. So we get:

$$
\lim _{h \rightarrow 0}(\lambda(h) / h)=\Theta_{0} b .
$$

Remark 4.5. - As in [12], this leads to localization theorems ([23,13], see also [28]). In particular, if the magnetic field is constant, then the ground state is localized near the points of the boundary where the magnetic field is parallel to the tangent space.

Application. An interesting case is the case when $H$ is constant and when $\Omega$ is the ellipsoid:

$$
a_{1} x_{1}^{2}+a_{2} x_{2}^{2}+a_{3} x_{3}^{2} \leqslant 1 .
$$

The set of points where $\vec{H}=\left(H_{1}, H_{2}, H_{3}\right)$ is parallel is obtained by intersecting the boundary of the ellipsoid with the plane:

$$
a_{1} x_{1} H_{1}+a_{2} x_{2} H_{2}+a_{3} x_{3} H_{3}=0 .
$$

More generally, if the surface is strictly convex and if the magnetic field is constant, it is possible to show that the set of points of the boundary where $H$ is parallel to the tangent space is a $C^{\infty}$ curve.

We emphasize that Theorem 4.4 does not explain all the situation. In the case with constant magnetic field it would be nice to show the role of some curvature in the localization as in the case of dimension 2. This is actually our goal to give an answer to this question.

\section{Adapted coordinates}

\subsection{Magnetic geometrical invariants}

The standard coordinates on $\mathbb{R}^{3}$ will be denoted by $x=\left(x_{1}, x_{2}, x_{3}\right)$ and the standard flat metric by $g_{0}$. We will also use $\langle$.$\left.| . \right\rangle$ for $g_{0}(.,$.$) or for g_{0}^{\star}(.,$.$) , and |X|$ for $(\langle X \mid X\rangle)^{1 / 2}$, (if $X$ is a vector field or a one-form). The standard volume on $\mathbb{R}^{3}$ will be denoted by

$$
\omega_{3}=d x^{3}=d x_{1} \wedge d x_{2} \wedge d x_{3}
$$

and will fix also the orientation of $\mathbb{R}^{3}$.

Let $A$ be a smooth magnetic potential one-form on $\bar{\Omega}$ :

$$
A=\sum_{j=1}^{3} A_{j}(x) d x_{j}, \quad A_{j} \in C^{\infty}(\bar{\Omega} ; \mathbb{R}) .
$$

The magnetic field $B$ is the two-form:

$$
B=d A=\sum_{1 \leqslant i<j \leqslant 3} B_{i j}(x) d x_{i} \wedge d x_{j}, \quad B_{i j}(x)=\frac{\partial A_{j}}{\partial x_{i}}(x)-\frac{\partial A_{i}}{\partial x_{j}}(x) .
$$

If needed $B_{i j}(x)$ is extended as an antisymmetric matrix $B_{x}$. The intensity of the magnetic field is the non-negative function

$$
|B|(x)=\sup \left\{\left|B_{x}(X, Y)\right| ; X, Y \in T_{x} \bar{\Omega},|X|=|Y|=1\right\}, \quad \forall x \in \bar{\Omega} .
$$


One associates to the magnetic field $B$, a vector field $\vec{H}:=H(B) \in T \bar{\Omega}$ by

$$
B_{x}(X, Y)=\omega_{3}\left(X, Y, H_{x}(B)\right), \quad \forall X, Y \in T_{x} \bar{\Omega} .
$$

In the initial coordinates, we have:

$$
H(B)=\sum_{j=1}^{3} b_{j}(x) \frac{\partial}{\partial x_{j}}, \quad \text { with } b_{1}=B_{23}, b_{2}=B_{31} \text { and } b_{3}=B_{12} .
$$

Then the magnetic intensity satisfies:

$$
|B|=|H(B)| \text {. }
$$

We associate to the magnetic potential $A$ the sesquilinear form on $H^{1}(\Omega)$ :

$$
u \mapsto q_{A}^{h}(u)=\int_{\Omega}|\sqrt{-1} h d u+u A|^{2} d x^{3} .
$$

Here $h \in] 0,+\infty[$ is a parameter. The associated differential operator is the magnetic Laplacian which is given in the standard coordinates by

$$
P_{A}^{h}=(h D-A)^{2}=\sum_{j=1}^{3}\left(h D_{x_{j}}-A_{j}(x)\right)^{2} .
$$

\subsection{Local coordinates}

If $y=\left(y_{1}, y_{2}, y_{3}\right)$ are new local coordinates, that is, if $\mathcal{V}_{x_{0}}$ is a neighborhood of some point $x_{0} \in \Omega$ and if $x \mapsto y=\Phi(x)$ is a diffeomorphism of $\mathcal{V}_{x_{0}}$ onto $\mathcal{O} \subset \mathbb{R}^{3}$, then in the new basis $\left(\frac{\partial}{\partial y_{1}}, \frac{\partial}{\partial y_{2}}, \frac{\partial}{\partial y_{3}}\right)$ of $T \mathcal{V}_{x_{0}}$, the standard metric $g_{0}$ and the volume $\omega_{3}$ become

$$
g_{0}=\sum_{1 \leqslant i, j \leqslant 3} g_{i j} d y_{i} \otimes d y_{j}
$$

and

$$
\omega_{3}=|g|^{1 / 2} d y_{1} \wedge d y_{2} \wedge d y_{3},
$$

with

$$
g_{i j}=\left\langle\frac{\partial x}{\partial y_{i}} \mid \frac{\partial x}{\partial y_{j}}\right\rangle, \quad\langle X \mid Y\rangle=\sum_{1 \leqslant i, j \leqslant 3} g_{i j} \widetilde{X}_{i} \widetilde{Y}_{j}, \quad|g|=\operatorname{det}\left(g_{i j}\right)
$$

where

$$
X=\sum_{j} \tilde{X}_{j} \frac{\partial}{\partial y_{j}} \quad \text { and } \quad Y=\sum_{j} \tilde{Y}_{j} \frac{\partial}{\partial y_{j}} .
$$

The magnetic potential is given in the new coordinates by

$$
A=\sum_{j=1}^{3} \widetilde{A}_{j} d y_{j} \quad \text { with } \widetilde{A}_{j}=\sum_{k=1}^{3} A_{k} \frac{\partial x_{k}}{\partial y_{j}} .
$$


The magnetic field is also given by

$$
B=\sum_{1 \leqslant i<j \leqslant 3} \widetilde{B}_{i j} d y_{i} \wedge d y_{j} \quad \text { with } \widetilde{B}_{i j}=\frac{\partial \widetilde{A}_{j}}{\partial y_{i}}-\frac{\partial \widetilde{A}_{i}}{\partial y_{j}} .
$$

So the vector magnetic field becomes

$$
H(B)=\sum_{j=1}^{3} \tilde{b}_{j} \frac{\partial}{\partial y_{j}}
$$

with

$$
\tilde{b}_{1}=|g|^{-1 / 2} \widetilde{B}_{23}, \quad \tilde{b}_{2}=|g|^{-1 / 2} \widetilde{B}_{31}, \quad \tilde{b}_{3}=|g|^{-1 / 2} \widetilde{B}_{12},
$$

and the intensity of the magnetic field becomes:

$$
|B|=\left(\sum_{i, j} g_{i j} \tilde{b}_{i} \tilde{b}_{j}\right)^{1 / 2}
$$

The sesquilinear form takes the following form, for $u$ supported in $\mathcal{V}_{x_{0}}$,

$$
q_{A}^{h}(u)=\int_{\mathcal{V}_{x_{0}}}|g|^{1 / 2} \sum_{1 \leqslant i, j \leqslant 3} g^{i j}\left[h D_{y_{i}} u-\widetilde{A}_{i} u\right] \times \overline{\left[h D_{y_{j}} u-\widetilde{A}_{j} u\right]} d y^{3},
$$

and the associated differential operator is

$$
P_{A}^{h}=|g|^{-1 / 2} \sum_{1 \leqslant i, j \leqslant 3}\left(h D_{y_{j}}-\widetilde{A}_{j}\right) \cdot|g|^{1 / 2} g^{i j}\left(h D_{y_{i}}-\widetilde{A}_{i}\right) .
$$

Here $g^{i j}$ is the inverse matrix of the matrix $g_{i j}$.

\subsection{Adapted local coordinates near the boundary}

Let $\phi(x)=\left(y_{1}, y_{2}\right)$ be local coordinates on the boundary and $G$ the induced metric by $g_{0}$ on $\partial \Omega$ in these coordinates. Then for $\varepsilon>0$ small enough (and modifying a little $\mathcal{V}_{x_{0}}$ if necessary), we can define local coordinates on $\mathcal{V}_{x_{0}}$,

$$
\left.\Phi: \mathcal{V}_{x_{0}} \rightarrow \mathcal{S} \times\right] 0, \varepsilon\left[, \quad \Phi(x)=\left(y_{1}, y_{2}, y_{3}\right),\right.
$$

where $\mathcal{S}$ is an open set in $\mathbb{R}^{2}$, such that

$$
y_{3}(x)=\operatorname{dist}\left(x, \phi^{-1}\left(y_{1}, y_{2}\right)\right)=\operatorname{dist}(x, \partial \Omega) ;
$$

so

$$
x=\phi^{-1}\left(y_{1}, y_{2}\right)+y_{3} N\left(\phi^{-1}\left(y_{1}, y_{2}\right)\right), \quad \forall x \in \mathcal{V}_{x_{0}},
$$

where $N(x)$ is the interior unit normal to $\partial \Omega$ at the point $x \in \partial \Omega$.

Then we get by simple computation the form of the standard flat metric $g_{0}$ in these new coordinates, 


$$
\begin{aligned}
g_{0}= & \sum_{1 \leqslant i, j \leqslant 3} g_{i j} d y_{i} \otimes d y_{j} \\
= & d y_{3} \otimes d y_{3}+G+2 y_{3} \sum_{1 \leqslant i, j \leqslant 2}\left\langle\frac{\partial N}{\partial y_{i}} \mid \frac{\partial x}{\partial y_{j}}\right\rangle d y_{i} \otimes d y_{j} \\
& +y_{3}^{2} \sum_{1 \leqslant i, j \leqslant 2}\left\langle\frac{\partial N}{\partial y_{i}} \mid \frac{\partial N}{\partial y_{j}}\right\rangle d y_{i} \otimes d y_{j} .
\end{aligned}
$$

In this case we have, using (5.10) and (5.18),

$$
\widetilde{A}_{3}=\sum_{j=1}^{3} A_{j} N_{j}
$$

Remark 5.1. - In the following, we choose a finite family of open sets $\mathcal{V}_{x_{j}}\left(x_{j} \in \partial \Omega\right)$ covering a tubular neighborhood of $\partial \Omega$. When we speak later of local coordinates, we mean that we take some element belonging to this family.

\section{Rough upper bounds}

To prove our results, we need some rough preliminary estimates (with some control of the remainder) of the ground state energy.

Proposition 6.1. - Let $\Omega$ be a bounded open set of $\mathbb{R}^{3}$ with smooth boundary $\partial \Omega$. Let $P_{A, \Omega}^{h, N}$ be the Neumann operator on $L^{2}(\Omega)$ associated to the Schrödinger operator with constant magnetic field $(h D-A)^{2}$ and let us assume that the vector magnetic field $H=\operatorname{curl}(A)$ is constant of intensity $b$.

If $\lambda(h)=\inf \operatorname{Sp}\left(P_{A, \Omega}^{h, N}\right)$ is the first eigenvalue of $P_{A, \Omega}^{h, N}$, then there exists a constant $C_{0}$ such that

$$
\lambda(h) \leqslant b \Theta_{0} h+C_{0} h^{4 / 3} .
$$

Remark 6.2. - It is not necessary to assume that the magnetic field is constant as the proof of this proposition will show. If one choose a point $x_{0}$ such that $H\left(x_{0}\right)$ is tangent to $\partial \Omega$, one can get the same result with $b=\left|H\left(x_{0}\right)\right|$. One can then optimize by choosing a point of this kind such that $\left|H\left(x_{0}\right)\right|$ is minimal. If $\Gamma_{H}$ is the set introduced in (1.4) we will prove:

$$
\lambda(h) \leqslant \Theta_{0} \inf _{x \in \Gamma_{H}}|H(x)| h+C_{0} h^{4 / 3} .
$$

This kind of estimate, with a more explicit, but not optimal $C_{0}$, appears already in the appendix of [23].

Proof of Proposition 6.1. - As the flux of the magnetic field through the boundary is zero,

$$
\int_{\partial \Omega}\langle\operatorname{curl}(A(x)) \mid N(x)\rangle d s=\int_{\Omega} \operatorname{div} \operatorname{curl}(A) d x=0,
$$

if $N$ is the interior normal unit of $\partial \Omega$, there exists $x_{0} \in \partial \Omega$ such that the vector magnetic field is tangent to $\partial \Omega$ at $x_{0}$ :

$$
\left\langle\operatorname{curl}\left(A\left(x_{0}\right)\right) \mid N\left(x_{0}\right)\right\rangle=0 .
$$


We take local coordinates $\left(y_{1}, y_{2}\right)$ in a neighbourhood of $W_{0}$ of $x_{0}$ in $\partial \Omega$ such that the metric is $\delta_{i j}$ at $x_{0}$ and $\frac{\partial}{\partial y_{1}}$ is parallel to the vector magnetic field.

We consider the adapted coordinates $y=\left(y_{1}, y_{2}, y_{3}\right)$, with $y_{3}(x)=d(x, \partial \Omega)$, and then $g_{i j}\left(x_{0}\right)=\delta_{i j}$. If $\widetilde{A}$ is the magnetic potential in the new coordinates, $A \cdot d x=\widetilde{A} \cdot d y$, then

$$
\begin{aligned}
& \frac{\partial \widetilde{A}_{3}}{\partial y_{2}}\left(x_{0}\right)-\frac{\partial \widetilde{A}_{2}}{\partial y_{3}}\left(x_{0}\right)=b, \\
& \frac{\partial \widetilde{A}_{1}}{\partial y_{3}}\left(x_{0}\right)-\frac{\partial \widetilde{A}_{3}}{\partial y_{1}}\left(x_{0}\right)=0, \\
& \left.\frac{\partial \widetilde{A}_{2}}{\partial y_{1}}\left(x_{0}\right)-\frac{\partial \widetilde{A}_{1}}{\partial y_{2}}\left(x_{0}\right)\right)=0 .
\end{aligned}
$$

We can find a gauge transform $\exp i \frac{\varphi}{h}$, with $\varphi$ real, such that, in (8.27),

$$
\widetilde{A}_{1}\left(x_{0}\right)=0, \quad \widetilde{A}_{2}\left(x_{0}\right)=0, \quad \widetilde{A}_{3}=0 .
$$

We first estimate the error occuring when eliminating the terms vanishing to order 3 .

If $u \in H^{1}(\Omega)$ is such that

$$
\operatorname{supp}(u) \subset\left\{x ;\left|x-x_{0}\right| \leqslant h^{\delta}\right\}
$$

with $\delta>0$, and if $h$ is small enough, then, for some $C>0$,

$$
q_{A}^{h}\left(e^{i \frac{\varphi}{h}} u\right) \leqslant\left(1+h^{\delta} C\right) q_{A^{0}}^{h}(u)+C\left[h^{3 \delta}\left(q_{A^{0}}^{h}(u)\right)^{1 / 2} \cdot\|u\|+h^{6 \delta}\|u\|^{2}\right]
$$

where

$$
A_{1}^{0}=R_{1}(y), \quad A_{2}^{0}=-b y_{3}+R_{2}(y), \quad A_{3}^{0}=0,
$$

the $R_{j}(y)$ are polynomial functions, homogeneous of order two,

$$
R_{j}(y)=\sum_{|\alpha|=2} a_{j, \alpha} y^{\alpha}
$$

and

$$
q_{A^{0}}^{h}(u)=\int_{|y| \leqslant C h^{\delta}, y_{3}>0} \mid\left(h D_{y} u-\left.A^{0}(y) u\right|^{2} d y .\right.
$$

We take the function $u$ in the form

$$
u\left(y_{1}, y_{2}, y_{3}\right):=e^{-i b^{1 / 2} y_{2} \xi_{0} / h^{1 / 2}} v(y)
$$

with

$$
v\left(y_{1}, y_{2}, y_{3}\right)=h^{-\frac{1}{4}-\delta} \varphi_{0}\left(b^{1 / 2} h^{-1 / 2} y_{3}\right) \chi\left(4 h^{-\delta} y_{3}\right) \chi\left(4 h^{-\delta}\left(y_{1}^{2}+y_{2}^{2}\right)^{1 / 2}\right) .
$$

Here $\chi$ is a cut off function equal to one on $\left[-\frac{1}{2}, \frac{1}{2}\right]$ and supported in $[-1,1], \varphi_{0}$ is introduced in Section 2.2 and $\delta \in] 0, \frac{1}{2}[$. 
Observing that:

$$
\int_{\mathbb{R}_{+}}\left(\left|D_{t} \varphi_{0}(t)\right|^{2}+\left|\left(t-\xi_{0}\right) \varphi_{0}(t)\right|^{2}\right) d t=\Theta_{0} \int_{\mathbb{R}_{+}}\left|\varphi_{0}(t)\right|^{2} d t,
$$

and that $t \mapsto \varphi_{0}(t)$ is an exponentially decreasing function at infinity, we get when $\delta=\frac{1}{3}$,

$$
q_{\hat{A}^{0}}^{h}(u) \leqslant\left(b \Theta_{0} h+C h^{4 / 3}\right)\|u\|^{2},
$$

where

$$
\hat{A}_{1}^{0}=0, \quad \hat{A}_{2}^{0}=-b y_{3}, \quad \hat{A}_{3}^{0}=0 .
$$

We then have to compare $q_{\hat{A}^{0}}^{h}(u)$ and $q_{A^{0}}^{h}(u)$. The first try could be to use:

$$
q_{A^{0}}^{h}\left(e^{i \frac{\varphi}{h}} u\right) \leqslant\left(1+h^{\delta} C\right) q_{\hat{A}^{0}}^{h}(u)+C\left[h^{2 \delta}\left(q_{\hat{A}^{0}}^{h}(u)\right)^{1 / 2} \cdot\|u\|+h^{4 \delta}\|u\|^{2}\right] .
$$

This leads to error terms of size $h^{1+\delta}, h^{\frac{1}{2}+2 \delta}, h^{4 \delta}$ and $h^{2-2 \delta}$. But this leads only, using (6.9) and (6.10) with $\delta=\frac{1}{3}$, to (6.1) with $\mathcal{O}\left(h^{7 / 6}\right)$ instead of $\mathcal{O}\left(h^{4 / 3}\right)$ as expected.

In order to get effectively (6.1), we need to use (2.8). We have indeed to analyze more carefully the term which was bounded from above in (6.10) by $h^{2 \delta}\left(q_{\hat{A}^{0}}^{h}(u)\right)^{1 / 2} \cdot\|u\|$.

The terms which were estimated by $h^{\frac{1}{2}+2 \delta}$ are of the form

$$
h^{-\frac{1}{2}-2 \delta} \int y_{j} y_{k}\left(\xi_{0}-\frac{b^{1 / 2} y_{3}}{h^{1 / 2}}\right) \varphi_{0}^{2}\left(b^{1 / 2} h^{-1 / 2} y_{3}\right) \chi^{2}\left(4 h^{-\delta}\left(y_{1}^{2}+y_{2}^{2}\right)^{1 / 2}\right) d y
$$

with $j$ and $k$ equal to 1 or 2 . But they actually vanish due to (2.8).

The other terms have actually better upper bounds. For example:

$$
h^{-\frac{1}{2}-2 \delta} \int y_{2} y_{3}\left(\xi_{0}-\frac{b^{1 / 2} y_{3}}{h^{1 / 2}}\right) \varphi_{0}^{2}\left(b^{1 / 2} h^{-1 / 2} y_{3}\right) \chi\left(4 h^{-\delta} y_{3}\right) \chi^{2}\left(4 h^{-\delta}\left(y_{1}^{2}+y_{2}^{2}\right)^{1 / 2}\right) d y
$$

can be estimated by $\mathcal{O}\left(h^{1+\delta}\right)$. This achieves the proof of Proposition 6.1.

This suggests strongly that the next term in the expansion of $\lambda(h)$ is $\mathcal{O}\left(h^{4 / 3}\right)$, but to go further we need to analyze the model more carefully in the neighborhood of the points of the boundary where $\langle H(x) \mid N(x)\rangle$ vanishes. For this we need to enter more deeply into the geometry of the boundary in connection with the magnetic vector field, and this will be done in Section 8.

\section{Rough lower bounds}

We assume that the magnetic field is constant. Let $u^{h}$ be an eigenfunction associated to the eigenvalue $\lambda(h)=\inf \operatorname{Sp}\left(P_{A, \Omega}^{h, N}\right)$.

\subsection{A priori estimates}

As proved in [12], we have, without the assumption (1.5), the following behavior of $u^{h}$. 
Proposition 7.1. - If $t=t(x)=d(x, \partial \Omega)$, then, for any $k \in \mathbb{N}$, there exists a constant $C_{k}$ depending only on $k$, such that

$$
\left\|t^{k / 2} u^{h}\right\| \leqslant C_{k} h^{k / 4}\left\|u^{h}\right\|
$$

and

$$
\left\|t^{k / 2}(h D-A) u^{h}\right\| \leqslant C_{k} h^{(k+2) / 4}\left\|u^{h}\right\| .
$$

Proof. - Proposition 6.1 and the fact that $\Theta_{0}<1$ give the existence of constants $h_{0}>0$ and $C_{0}>1$ such that

$$
\left.\left.C_{0}^{-1} b h \leqslant b h-\lambda(h) \leqslant C_{0} b h, \quad \forall h \in\right] 0, h_{0}\right] .
$$

Of course this is a very rough estimate.

Let us remark that (7.1) and (7.2) are valid when $k=0$. As for the 2-dimensional case in [11], we proceed by recursion.

By changing $t$ away from the boundary, we can assume that $x \mapsto t(x)=d(x, \partial \Omega)$ is extended as a $C^{1}(\bar{\Omega})$ function. By choosing suitable coordinates and after a gauge transform, we can assume that

$$
A_{1}(x)=0, \quad A_{2}(x)=-\frac{b}{2} x_{3}, \quad A_{3}(x)=\frac{b}{2} x_{2} .
$$

As $t / \partial \Omega=0$, we have by integrating by parts and for $k>0$,

$$
\begin{aligned}
i h b \int_{\Omega} t^{k}\left|u^{h}\right|^{2} d x= & \int_{\Omega} t^{k}\left[\left(h D_{2}-A_{2}\right),\left(h D_{3}-A_{3}\right)\right] u^{h} \cdot \bar{u}^{h} d x \\
= & \int_{\Omega} t^{k}\left\{\left(h D_{3}-A_{3}\right) u^{h} \overline{\left(h D_{2}-A_{2}\right) u^{h}}-\left(h D_{2}-A_{2}\right) u^{h} \overline{\left(h D_{3}-A_{3}\right) u^{h}}\right\} d x \\
& +i h k \int_{\Omega} t^{k-1}\left\{\frac{\partial t}{\partial x_{2}}\left(h D_{3}-A_{3}\right) u^{h}-\frac{\partial t}{\partial x_{3}}\left(h D_{2}-A_{2}\right) u^{h}\right\} \bar{u}^{h} d x
\end{aligned}
$$

and

(7.5) $\int_{\Omega} t^{k}\left|(h D-A) u^{h}\right|^{2} d x=\int_{\Omega}\left\{\lambda(h) t^{k}\left|u^{h}\right|^{2}-i h k t^{k-1}\left(\left(\nabla_{x} t\right) \cdot(h D-A) u^{h}\right) \bar{u}^{h}\right\} d x$.

If $k=1$ we get from (7.4)

$$
h b \int_{\Omega} t\left|u^{h}\right|^{2} d x \leqslant \int_{\Omega} t\left|(h D-A) u^{h}\right|^{2} d x+C h\left\|(h D-A) u^{h}\right\| \cdot\left\|u^{h}\right\|,
$$

and then, we use (7.5) to get

$$
h b \int_{\Omega} t\left|u^{h}\right|^{2} d x \leqslant \lambda(h) \int_{\Omega} t\left|u^{h}\right|^{2} d x+C h\left\|(h D-A) u^{h}\right\| \cdot\left\|u^{h}\right\| .
$$

So

$$
(h b-\lambda(h)) \int_{\Omega} t\left|u^{h}\right|^{2} d x \leqslant C h \sqrt{\lambda(h)}\left\|u^{h}\right\|^{2} .
$$


We obtain (7.1) with $k=1$ from (7.3) and (7.8), and then it is easy to get (7.2) for $k=1$ from (7.5) by using (7.3), (7.1) with $k=1$ and (7.2) with $k=0$.

If $k \geqslant 2$, we get in the same way

$$
(h b-\lambda(h)) \int_{\Omega} t^{k}\left|u^{h}\right|^{2} d x \leqslant h k C\left\|t^{-1+\frac{k}{2}}(h D-A) u^{h}\right\| \cdot\left\|t^{k / 2} u^{h}\right\|,
$$

which gives, using (7.3),

$$
\left\|t^{k / 2} u^{h}\right\| \leqslant C k\left\|t^{-1+\frac{k}{2}}(h D-A) u^{h}\right\| .
$$

Using (7.10) and (7.5) we get

$$
\left\|t^{k} \mid(h D-A) u^{h}\right\|^{2} \leqslant C k h\left[\left\|t^{k / 2} u^{h}\right\|^{2}+\left\|t^{-1+\frac{k}{2}} \mid(h D-A) u^{h}\right\|^{2}\right] .
$$

Then we can proceed by recursion. (7.2) for $k=j-2$ and (7.10) for $k=j$ give (7.1) for $k=j$. Formulas (7.11) with $k=j$ and (7.2) for $k=j-2$ give (7.2) with $k=j$.

\subsection{A partition of unity}

Let $\left(\chi_{\gamma}(z)\right)_{\gamma \in \mathbb{Z}^{3}}$ be a partition of unity of $\mathbb{R}^{3}$. For example we can take

$$
\chi_{\gamma} \in C^{\infty}\left(\mathbb{R}^{3} ; \mathbb{R}\right) \quad \text { and } \operatorname{supp}\left(\chi_{\gamma}\right) \subset \gamma+[-1,1]^{3}, \quad \forall \gamma \in \mathbb{Z}^{3},
$$

$$
\sum_{\gamma} \chi_{\gamma}^{2}(z)=1 \text { and } \sum_{\gamma}\left|\nabla \chi_{\gamma}(z)\right|^{2}<\infty .
$$

If $\tau(h)$ is a function of $h$ such that $\tau(h) \in] 0, \varepsilon(\Omega)[$, where $\varepsilon(\Omega)$ is the geometric constant which is the maximal $\varepsilon$ for the property that $\{d(x, \partial \Omega)<\varepsilon\}$ is a regular tubular neighborhood in $\bar{\Omega}$ of $\partial \Omega$, we will define the functions

$$
\chi_{\gamma, \tau(h)}(z)=\chi_{\gamma}(z / \tau(h)), \quad \forall \gamma \in \mathbb{Z}^{3} .
$$

So we get a new partition of unity such that

$$
\sum_{\gamma} \chi_{\gamma, \tau(h)}^{2}(z)=1, \quad \sum_{\gamma}\left|\nabla \chi_{\gamma, \tau(h)}(z)\right|^{2} \leqslant C \tau(h)^{-2},
$$

and

$$
\operatorname{supp}\left(\chi_{\gamma, \tau(h)}\right) \subset \tau(h) \gamma+[-\tau(h), \tau(h)]^{3} .
$$

Then, for any $u \in H^{1}(\Omega)$, we have:

$$
q_{A}^{h}(u)=\sum_{\gamma}\left[q_{A}^{h}\left(\chi_{\gamma, \tau(h)} u\right)-h^{2}\left\|\left|\nabla \chi_{\gamma, \tau(h)}\right| u\right\|^{2}\right] .
$$

Let us define 


$$
\begin{aligned}
& \Gamma_{\tau(h)}(\Omega)=\left\{\gamma \in \mathbb{Z}^{3} ; \operatorname{supp}\left(\chi_{\gamma, \tau(h)}\right) \cap \Omega \neq \emptyset\right\}, \\
& \Gamma_{\tau(h)}^{0}(\Omega)=\left\{\gamma \in \Gamma_{\tau(h)}(\Omega) ; \operatorname{dist}\left(\operatorname{supp}\left(\chi_{\gamma, \tau(h)}\right), \partial \Omega\right)>\tau(h)\right\}, \\
& \Gamma_{\tau(h)}^{1}(\Omega)=\left\{\gamma \in \Gamma_{\tau(h)}(\Omega) ; \operatorname{dist}\left(\operatorname{supp}\left(\chi_{\gamma, \tau(h)}\right), \partial \Omega\right) \leqslant \tau(h)\right\} .
\end{aligned}
$$

\subsection{Proof of Theorem 1.1: lower bounds}

For the moment, we give a rough lower bound of the ground state energy in the case of constant magnetic field.

Proposition 7.2. - Under the assumptions of Proposition 6.1, there exists $C_{0}$ such that:

$$
b \Theta_{0} h-C_{0} h^{4 / 3} \leqslant \lambda(h) .
$$

Proof. - We proceed as in the proof of Proposition 9.2 of [12] and use the partition of unity introduced in the previous subsection.

Far from the boundary. As $\operatorname{curl} A$ is constant, the standard estimate gives:

$$
\|(h D-A) w\|_{L^{2}\left(\mathbb{R}^{3}\right)}^{2} \geqslant b h\|w\|_{L^{2}\left(\mathbb{R}^{3}\right)}^{2}, \quad \forall w \in C_{0}^{\infty}\left(\mathbb{R}^{3}\right) .
$$

So

$$
q_{A}^{h}\left(\chi_{\gamma, \tau(h)} u\right) \geqslant b h\left\|\chi_{\gamma, \tau(h)} u\right\|^{2}, \quad \forall \gamma \in \Gamma_{\tau(h)}^{0}(\Omega) .
$$

Near the boundary. Suppose $\gamma \in \Gamma_{\tau(h)}^{1}(\Omega)$; then there exist local coordinates ${ }^{4}$ adapted to the boundary $y=\left(y_{1}, y_{2}, y_{3}\right)$ such that $y_{3}(x)=d(x, \partial \Omega)$.

Then, for $\tau(h)<1$ and for some integer $k \in \mathbb{N}^{\star}$,

$$
\begin{aligned}
q_{A}^{h}\left(\chi_{\gamma, \tau(h)} u\right) \geqslant & (1-C \tau(h)) q_{\widetilde{A}^{(k)}}^{h}\left(\chi_{\gamma, \tau(h)} u\right) \\
& -C(\tau(h))^{k+1}\left\|\chi_{\gamma, \tau(h)} u\right\| \cdot\left(q_{A}^{h}\left(\chi_{\gamma, \tau(h)} u\right)\right)^{1 / 2} \\
& -C(\tau(h))^{2 k+2}\left\|\chi_{\gamma, \tau(h)} u\right\|^{2}
\end{aligned}
$$

with, for some $y_{0} \in \partial \Omega \cap\left\{[-\tau(h), \tau(h)]^{3}+\operatorname{supp}\left(\chi_{\gamma, \tau(h)}\right)\right\}$,

$$
\widetilde{A}^{(k)}=\widetilde{A}\left(y_{0}\right)+\sum_{1 \leqslant|\alpha| \leqslant k}\left(y-y_{0}\right)^{\alpha} \frac{\partial^{\alpha} \widetilde{A}}{\partial y^{\alpha}}\left(y_{0}\right)
$$

and

$$
\begin{aligned}
q_{\widetilde{A}^{(k)}}^{h}(w)= & |g|^{1 / 2}\left(y_{0}\right) \int_{\mathbb{R}^{2} \times \mathbb{R}_{+}}\left[\left|\left(h D_{y_{3}}-\widetilde{A}_{3}^{(k)}\right) w\right|^{2}\right. \\
& \left.+\sum_{1 \leqslant i, j \leqslant 2} g^{i j}\left(y_{0}\right)\left(h D_{y_{i}}-\widetilde{A}_{i}^{(k)}\right) w \cdot \overline{\left(h D_{y_{j}}-\widetilde{A}_{j}^{(k)}\right) w}\right] d y .
\end{aligned}
$$

Let us remark that

$$
\left\|\chi_{\gamma, \tau(h)} u\right\|^{2} \leqslant(1+C \tau(h))|g|^{1 / 2}\left(y_{0}\right) \int_{\mathbb{R}^{2} \times \mathbb{R}_{+}}\left|\chi_{\gamma, \tau(h)} u\right|^{2} d y,
$$

\footnotetext{
${ }^{4}$ Belonging to the family introduced in Section 5.3.
} 
and by (5.12)

$$
b^{2}=|g|^{-1}\left(y_{0}\right)\left[\left(\widetilde{H}_{3}^{0}\right)^{2}+\sum_{1 \leqslant j, k \leqslant 2} g^{j k}\left(y_{0}\right) \widetilde{H}_{j}^{0} \widetilde{H}_{k}^{0}\right]
$$

if

$$
\begin{aligned}
\widetilde{H}_{1}^{0} & =\frac{\partial \widetilde{A}_{3}}{\partial y_{2}}\left(y_{0}\right)-\frac{\partial \widetilde{A}_{2}}{\partial y_{3}}\left(y_{0}\right), \\
\widetilde{H}_{2}^{0} & =\frac{\partial \widetilde{A}_{1}}{\partial y_{3}}\left(y_{0}\right)-\frac{\partial \widetilde{A}_{3}}{\partial y_{1}}\left(y_{0}\right), \\
\widetilde{H}_{3}^{0} & =\frac{\partial \widetilde{A}_{2}}{\partial y_{1}}\left(y_{0}\right)-\frac{\partial \widetilde{A}_{1}}{\partial y_{2}}\left(y_{0}\right) .
\end{aligned}
$$

We observe that by (3.3)

$$
\sin \vartheta\left(y_{0}\right)=-|g|^{-1 / 2}\left(y_{0}\right) \widetilde{H}_{3}^{0} / b .
$$

So, the study of the constant magnetic field in a half-space done in Section 2, (more precisely (3.10), property 7 in Section 3.3 and Theorem 3.11), (7.23) and (7.24) show that there exists $\varepsilon_{1}>0$, such that for all $w \in C^{\infty}\left(\overline{\mathbb{R}_{+}^{3}}\right)$ with compact support,

$$
q_{\widetilde{A}^{(1)}}^{h}(w) \geqslant b h\left(\Theta_{0}+\varepsilon_{1}|g|^{-1 / 2}\left(y_{0}\right) \frac{\left|\widetilde{H}_{3}^{0}\right|}{b}\right)|g|^{1 / 2}\left(y_{0}\right) \int_{\mathbb{R}^{2} \times \mathbb{R}_{+}}|w|^{2} d y .
$$

We will consider two cases. Let $C_{1}>1$ to be chosen later. For more simplicity, we can assume, after a rotation and a dilation in $\left(y_{1}, y_{2}\right)$, that the metric $g$ is standard at $y_{0}$ :

$$
g\left(y_{0}\right)=\left(\delta_{i j}\right)
$$

First case: $\frac{\left|\widetilde{H}_{3}^{0}\right|}{b} \geqslant \frac{C_{1}}{\varepsilon_{1}} h^{-1 / 2} \tau(h)^{2}$.

We take $k=1$. Then (7.19), (7.22) and (7.25) prove that

$$
\begin{aligned}
& q_{A}^{h}\left(\chi_{\gamma, \tau(h)} u\right)+C(\tau(h))^{2}\left\|\chi_{\gamma, \tau(h)} u\right\| \cdot\left(q_{A}^{h}\left(\chi_{\gamma, \tau(h)} u\right)\right)^{1 / 2} \\
& \quad \geqslant\left[b h\left(\Theta_{0}+C_{1} h^{-1 / 2} \tau(h)^{2}\right)-C\left(h \tau(h)+(\tau(h))^{4}\right)\right] \cdot\left\|\chi_{\gamma, \tau(h)} u\right\|^{2} .
\end{aligned}
$$

This implies:

$$
\begin{aligned}
& \left(\left(q_{A}^{h}\left(\chi_{\gamma, \tau(h)} u\right)\right)^{1 / 2}+C \tau(h)^{2}\left\|\chi_{\gamma, \tau(h)} u\right\|\right)^{2} \\
& \quad \geqslant b h\left(\Theta_{0}+C_{1} h^{-1 / 2} \tau(h)^{2}\right) \cdot(1-C \tau(h))\left\|\chi_{\gamma, \tau(h)} u\right\|^{2} .
\end{aligned}
$$

Taking the square root, we get:

$$
\begin{aligned}
& \left(q_{A}^{h}\left(\chi_{\gamma, \tau(h)} u\right)\right)^{1 / 2} \\
& \quad \geqslant\left((b h)^{1 / 2}\left(\Theta_{0}+C_{1} h^{-1 / 2} \tau(h)^{2}\right)^{1 / 2} \cdot(1-C \tau(h))-C \tau(h)^{2}\right)\left\|\chi_{\gamma, \tau(h)} u\right\| .
\end{aligned}
$$

This finally gives, if $C_{1}$ is large enough and if, for some $\varepsilon_{0}>0$, the weight $\tau(h)$ satisfies the condition $h^{1 / 4+\varepsilon_{0}} \geqslant \tau(h) \geqslant h^{1 / 2}$,

$$
q_{A}^{h}\left(\chi_{\gamma, \tau(h)} u\right) \geqslant h\left(b\left(\Theta_{0}+\frac{1}{2} C_{1} h^{-1 / 2} \tau(h)^{2}\right)-C \tau(h)\right)\left\|\chi_{\gamma, \tau(h)} u\right\|^{2} .
$$


So if $h^{1 / 4+\varepsilon_{0}} \geqslant \tau(h) \geqslant h^{1 / 2}$ and $C_{1}$ is large enough, there exists $h_{0}>0$ such that

$$
\left.\left.q_{A}^{h}\left(\chi_{\gamma, \tau(h)} u\right) \geqslant b h \Theta_{0}\left\|\chi_{\gamma, \tau(h)} u\right\|^{2}, \quad \forall h \in\right] 0, h_{0}\right] .
$$

We now keep $C_{1}$ fixed such that (7.31) is satisfied.

Second case: $\frac{\left|\widetilde{H}_{3}^{0}\right|}{b}<\frac{C_{1}}{\varepsilon_{1}} h^{-1 / 2} \tau(h)^{2}$.

We proceed by steps:

Step 1: We use (7.23) but we take $k=2$. This leads to:

$$
\begin{aligned}
q_{A}^{h}\left(\chi_{\gamma, \tau(h)} u\right) \geqslant & (1-C \tau(h)) q_{\widetilde{A}^{(k)}}^{h}\left(\chi_{\gamma, \tau(h)} u\right) \\
& -C(\tau(h))^{3}\left\|\chi_{\gamma, \tau(h)} u\right\| \cdot\left(q_{A}^{h}\left(\chi_{\gamma, \tau(h)} u\right)\right)^{1 / 2} \\
& -C(\tau(h))^{4}\left\|\chi_{\gamma, \tau(h)} u\right\|^{2} .
\end{aligned}
$$

Step 2: Let us now remark that

$$
\begin{aligned}
q_{\widetilde{A}^{(2)}}^{h}\left(\chi_{\gamma, \tau(h)} u\right) \geqslant & q_{\widetilde{A}^{(2,1)}}^{h}\left(\chi_{\gamma, \tau(h)} u\right) \\
& -C\left[\tau(h)\left\|y_{3} \chi_{\gamma, \tau(h)} u\right\|+\left\|y_{3}^{2} \chi_{\gamma, \tau(h)} u\right\|\right] \cdot\left(q_{A}^{h}\left(\chi_{\gamma, \tau(h)} u\right)\right)^{1 / 2} \\
& -C\left[\tau(h)^{2}\left\|y_{3} \chi_{\gamma, \tau(h)} u\right\|^{2}+\left\|y_{3}^{2} \chi_{\gamma, \tau(h)} u\right\|^{2}\right]
\end{aligned}
$$

with

$$
\begin{aligned}
\widetilde{A}^{(2,1)}= & \widetilde{A}\left(y_{0}\right)+\sum_{j=1}^{3}\left(y_{j}-y_{0 j}\right) \frac{\partial \widetilde{A}}{\partial y_{j}}\left(y_{0}\right) \\
& +\sum_{j=1}^{2}\left(y_{j}-y_{0 j}\right)^{2} \frac{\partial^{2} \widetilde{A}}{\partial y_{j}^{2}}\left(y_{0}\right)+2\left(y_{1}-y_{01}\right)\left(y_{2}-y_{02}\right) \frac{\partial^{2} \widetilde{A}}{\partial y_{1} \partial y_{2}}\left(y_{0}\right) .
\end{aligned}
$$

Step 3: But it is easy to find a real polynomial function $p(y)$ such that

$$
q_{\widetilde{A}^{(2,1)}}^{h}\left(\chi_{\gamma, \tau(h)} u\right)=q_{\widetilde{A}^{(2,1,0)}}^{h}\left(e^{i \frac{p}{h}} \chi_{\gamma, \tau(h)} u\right),
$$

with

$$
\begin{aligned}
& \widetilde{A}_{1}^{(2,1,0)}=\widetilde{H}_{2}^{0} y_{3}+a_{1} y_{2}^{2}+b_{1}(y), \\
& \widetilde{A}_{2}^{(2,1,0)}=-\widetilde{H}_{1}^{0} y_{3}+\widetilde{H}_{3}^{0} y_{1}+a_{2} y_{1}^{2}+b_{2}(y), \\
& \widetilde{A}_{3}^{(2,1,0)}=0,
\end{aligned}
$$

where:

$$
b_{1}(y)=c_{11} y_{1} y_{3}+c_{12} y_{2} y_{3}, \quad b_{2}(y)=c_{21} y_{1} y_{3}+c_{22} y_{2} y_{3} .
$$

Step 4: Modulo an error like in the right hand side of (7.33), we are reduced to the analysis of

$$
\begin{aligned}
& \widetilde{A}_{1}^{(2,1,1)}=\widetilde{H}_{2}^{0} y_{3}+a_{1} y_{2}^{2}, \\
& \widetilde{A}_{2}^{(2,1,1)}=-\widetilde{H}_{1}^{0} y_{3}+\widetilde{H}_{3}^{0} y_{1}+a_{2} y_{1}^{2}, \\
& \widetilde{A}_{3}^{(2,1,1)}=0 .
\end{aligned}
$$

We write 


$$
\begin{aligned}
q_{\widetilde{A}(2,1,1)}^{h}(w)= & h^{2}\left\|D_{y_{3}} w\right\|^{2}+\tilde{b}^{2}\left\|\left(y_{3}-\tilde{b}^{-1}\left(\sin \theta L_{1}-\cos \theta L_{2}\right)\right) w\right\|^{2} \\
& +\left\|\left(\cos \theta L_{1}+\sin \theta L_{2}\right) w\right\|^{2}
\end{aligned}
$$

with

$$
\tilde{b}=\left[\left(\widetilde{H}_{1}^{0}\right)^{2}+\left(\widetilde{H}_{2}^{0}\right)^{2}\right]^{1 / 2}, \quad \widetilde{H}_{1}^{0}=\tilde{b} \cos \theta, \quad \widetilde{H}_{2}^{0}=\tilde{b} \sin \theta,
$$

and

$$
L_{1}=h D_{y_{1}}-a_{1} y_{2}^{2}, \quad L_{2}=h D_{y_{2}}-\widetilde{H}_{3}^{0} y_{1}-a_{2} y_{1}^{2} .
$$

As the operator $\sin \theta L_{1}-\cos \theta L_{2}$, a priori defined on $\mathcal{S}\left(\mathbb{R}^{2}\right)$, has a self-adjoint realization on $L^{2}\left(\mathbb{R}^{2}\right)$. This is indeed, after rotation, an operator of the form $\frac{1}{i} \partial_{t}+\phi(t, y)$ which is unitary equivalent to $\frac{1}{i} \partial_{t}$. Hence, we can consider the spectral representation of $\sin \theta L_{1}-\cos \theta L_{2}$ and the analysis of Section 2.1 to see that

$$
h^{2}\left\|D_{y_{3}} w\right\|^{2}+\tilde{b}^{2}\left\|\left(y_{3}-\tilde{b}^{-1}\left(\sin \theta L_{1}-\cos \theta L_{2}\right)\right) w\right\|^{2} \geqslant h \tilde{b} \Theta_{0}\|w\|^{2} .
$$

Step 5: We now compare $b$ and $\tilde{b}$. We are in the case when $\frac{\left|\widetilde{H}_{3}^{0}\right|}{b}<C_{1} h^{-1 / 2} \tau(h)^{2}$. So

$$
|b-\tilde{b}| \leqslant C C_{1}^{2} h^{-1} \tau(h)^{4}
$$

Step 6: Then (7.32), (7.26) and (7.33)-(7.41) prove that, if $u^{h}$ is the eigenfunction associated to the ground state energy of $P_{A, \Omega}^{h, N}$ and if $\tau(h)=h^{1 / 3}$, then

$$
\begin{aligned}
q_{A}^{h}\left(\chi_{\gamma, \tau(h)} u^{h}\right) \geqslant & {\left[h b \Theta_{0}-C h^{4 / 3}\right]\left\|\chi_{\gamma, \tau(h)} u^{h}\right\|^{2} } \\
& -C h^{2 / 3}\left\|t \chi_{\gamma, \tau(h)} u^{h}\right\|^{2}-C\left\|t^{2} \chi_{\gamma, \tau(h)} u^{h}\right\|^{2} \\
& -C h^{1 / 3}\left\|t \chi_{\gamma, \tau(h)} u^{h}\right\|\left[q_{A}^{h}\left(\chi_{\gamma, \tau(h)} u^{h}\right)\right]^{1 / 2} \\
& -C\left\|t^{2} \chi_{\gamma, \tau(h)} u^{h}\right\|\left[q_{A}^{h}\left(\chi_{\gamma, \tau(h)} u^{h}\right)\right]^{1 / 2} .
\end{aligned}
$$

We now eliminate the two last lines at the price of a worse error term. We get first the existence of $C$ such that:

$$
\begin{aligned}
\left(1+C h^{1 / 3}\right) q_{A}^{h}\left(\chi_{\gamma, \tau(h)} u^{h}\right) \geqslant & \left(h b \Theta_{0}-C h^{4 / 3}\right)\left\|\chi_{\gamma, \tau(h)} u^{h}\right\|^{2} \\
& -C h^{1 / 3}\left\|t \chi_{\gamma, \tau(h)} u^{h}\right\|^{2}-C h^{-1 / 3}\left\|t^{2} \chi_{\gamma, \tau(h)} u^{h}\right\|^{2},
\end{aligned}
$$

which leads to the existence of $C$ such that:

$$
\begin{aligned}
q_{A}^{h}\left(\chi_{\gamma, \tau(h)} u^{h}\right) \geqslant & \left(h b \Theta_{0}-C h^{4 / 3}\right)\left\|\chi_{\gamma, \tau(h)} u^{h}\right\|^{2} \\
& -C h^{1 / 3}\left\|t \chi_{\gamma, \tau(h)} u^{h}\right\|^{2}-C h^{-1 / 3}\left\|t^{2} \chi_{\gamma, \tau(h)} u^{h}\right\|^{2} .
\end{aligned}
$$

The last two terms will be controlled by summation using Proposition 7.1.

End of the proof. With $\tau(h)=h^{1 / 3}$, we get from (7.31) and (7.44) that

$$
\begin{aligned}
q_{A}^{h}\left(\chi_{\gamma, \tau(h)} u^{h}\right) \geqslant & {\left[b \Theta_{0} h-C h^{4 / 3}\right] \cdot\left\|\chi_{\gamma, \tau(h)} u^{h}\right\|^{2} } \\
& -C h^{1 / 3}\left\|t \chi_{\gamma, \tau(h)} u^{h}\right\|^{2}-C h^{-1 / 3}\left\|t^{2} \chi_{\gamma, \tau(h)} u^{h}\right\|^{2},
\end{aligned}
$$

if $\gamma \in \Gamma_{\tau(h)}^{1}(\Omega)$. 
Using Proposition 7.1, we verify that:

$$
\sum_{\gamma \in \Gamma_{\tau(h)}^{1}}\left(h^{1 / 3}\left\|t \chi_{\gamma, \tau(h)} u^{h}\right\|^{2}+h^{-1 / 3}\left\|t^{2} \chi_{\gamma, \tau(h)} u^{h}\right\|^{2}\right) \leqslant C h^{4 / 3} .
$$

So we get

$$
\sum_{\gamma \in \Gamma_{\tau(h)}^{1}} q_{A}^{h}\left(\chi_{\gamma, \tau(h)} u^{h}\right) \geqslant\left(b \Theta_{0} h\right) \cdot \sum_{\gamma \in \Gamma_{\tau(h)}^{1}}\left\|\chi_{\gamma, \tau(h)} u^{h}\right\|^{2}-C h^{4 / 3}\left\|u_{h}\right\|^{2} .
$$

Then (7.17) is deduced from (7.13)-(7.16) with $\tau(h)=h^{1 / 3}$, (7.18) and from (7.47).

Proof of Theorem 1.1. - Theorem 1.1 follows from the lower bound (7.17) and the previously obtained upper bound (6.1).

\section{Refined adapted coordinates on the boundary and curvatures}

\subsection{Curvatures}

For the following geometric properties of embedded surface in $\mathbb{R}^{3}$, we refer mainly to the first chapter of [7] (see also the volume two of Spivak's book [31]). Let us suppose more specifically that $x_{0}$ is a point of the boundary $\partial \Omega$. The neighborhood of $x_{0} \mathcal{V}_{x_{0}}$ can be chosen such that, in $W_{x_{0}}:=\partial \Omega \cap \mathcal{V}_{x_{0}}$, there exist local coordinates $\left(y_{1}, y_{2}\right)$, i.e. there exist an open subset $\mathcal{S}$ of $\mathbb{R}^{2}$ and a diffeomorphism

$$
\phi: \partial \Omega \cap \mathcal{V}_{x_{0}} \rightarrow \mathcal{S}, \quad \phi(x)=\left(y_{1}, y_{2}\right) .
$$

We denote by $y \mapsto x(y)$ its inverse. Then $\left\{\frac{\partial x}{\partial y_{1}}, \frac{\partial x}{\partial y_{2}}\right\}$ is a basis of $T \mathcal{W}_{x_{0}}$.

The first fundamental form of $\partial \Omega$ is the restriction of $g_{0}$ to $\partial \Omega$ and it is denoted by $G$. In the local coordinates $\left(y_{1}, y_{2}\right), G$ is given by:

$$
G=\sum_{1 \leqslant i, j \leqslant 2} G_{i j} d y_{i} \otimes d y_{j}, \quad G_{i j}=\sum_{k=1}^{3} \frac{\partial x_{k}}{\partial y_{i}} \cdot \frac{\partial x_{k}}{\partial y_{j}} .
$$

The element of area is given by

$$
d s^{2}=|G|^{1 / 2} d y_{1} \wedge d y_{2},
$$

with

$$
|G|=\operatorname{det}\left(G_{i j}\right)_{1 \leqslant i, j \leqslant 2} .
$$

The unit normal vector to $\partial \Omega$ is defined by

$$
N=\frac{\frac{\partial x}{\partial y_{1}} \wedge \frac{\partial x}{\partial y_{2}}}{\left|\frac{\partial x}{\partial y_{1}} \wedge \frac{\partial x}{\partial y_{2}}\right|}
$$

For any vector fields $X$ and $Y, X \wedge Y$ is the vector field defined by

$$
\begin{aligned}
& \omega_{3}(X, Y, X \wedge Y)=|X|^{2} \cdot|Y|^{2}-\langle X \mid Y\rangle^{2}, \\
& \langle X \mid X \wedge Y\rangle=\langle Y \mid X \wedge Y\rangle=0 .
\end{aligned}
$$


When $\partial \Omega$ is the boundary of a bounded regular domain, we can choose moreover these coordinates such that $N$ is the interior normal unit vector, and we observe, using (8.4), that:

$$
\omega_{3}\left(\frac{\partial x}{\partial y_{1}}, \frac{\partial x}{\partial y_{2}}, N\right)>0
$$

Remembering that $|N|^{2}=1$, we get that, for $j=1,2$,

$$
\frac{\partial N}{\partial y_{j}} \in T \partial \Omega
$$

This permits us to introduce the Weingarten map $k$ from $T \partial \Omega$ into $T \partial \Omega$, by:

$$
k\left(\sum_{j=1}^{2} \lambda_{j} \frac{\partial x}{\partial y_{j}}\right)=-\sum_{j=1}^{2} \lambda_{j} \frac{\partial N}{\partial y_{j}} .
$$

This map is independent of the choice of the coordinates respecting (8.3) with $N$ inner normal.

The second fundamental form $K$ on $T \partial \Omega$ is defined, for $X$ and $Y$ in $T \partial \Omega$, by

$$
K(X, Y)=G(X, k(Y))=\sum_{1 \leqslant i, j \leqslant j} K_{i j} X_{i} Y_{j}
$$

where

$$
K_{i j}=-\left\langle\frac{\partial x}{\partial y_{i}} \mid \frac{\partial N}{\partial y_{j}}\right\rangle,
$$

and $X_{j}$ and $Y_{j}$ are the components of $X$ and $Y$ in the local basis $\left(\frac{\partial}{\partial y_{j}}\right)$.

Observing that $\left\langle\frac{\partial x}{\partial y_{i}} \mid N\right\rangle=0$, it is easy to see, by differentiating this equality, that

$$
K_{i j}=\left\langle\frac{\partial^{2} x}{\partial y_{i} \partial y_{j}} \mid N\right\rangle
$$

In particular $K$ is symmetric. We recall that the Gauss curvature is

$$
\kappa^{G}=\operatorname{det}(k)=\left(K_{11} K_{22}-K_{12}^{2}\right) /\left(G_{11} G_{22}-G_{12}^{2}\right),
$$

and that the mean curvature is

$$
\kappa^{M}=\frac{1}{2} \operatorname{tr}(k)=\frac{1}{2}\left(k_{11}+k_{22}\right)
$$

where $\left(k_{i j}\right)_{i j}$ is the matrix $G^{-1} K$.

Let $a<b$. If $[a, b] \ni \tau \mapsto \gamma(\tau) \in \partial \Omega$ defines a parametrized closed curve, we denote its oriented image by $\Gamma$. We assume moreover that it is parametrized by arc length, that is:

$$
\left|\gamma^{\prime}(\tau)\right|=1
$$

$4^{\mathrm{e}}$ SÉRIE - TOME $37-2004-\mathrm{N}^{\circ} 1$ 
The curvature vector $\left(\kappa_{g}, \kappa_{n}\right)$ of $\Gamma$ at the point $\gamma(\tau)$ is defined ${ }^{5}$ by

$$
\gamma^{\prime \prime}(\tau)=-\kappa_{g}(\gamma(\tau)) N(\gamma(\tau)) \wedge \gamma^{\prime}(\tau)+\kappa_{n}(\gamma(\tau)) N(\gamma(\tau))
$$

The function $\kappa_{g}$ is called the geodesic curvature and $\kappa_{n}$ the normal curvature of $\Gamma$, at the point $\gamma(\tau)$. We observe that this is well defined once an orientation of $\Gamma$ is chosen.

As shown in [7] (formula (18)), the normal curvature is given by the equation

$$
\kappa_{n}(\gamma(\tau))=K\left(\gamma^{\prime}(\tau), \gamma^{\prime}(\tau)\right)
$$

Remark 8.1. - Note (cf. [7]) that the geodesic curvature vanishes when $\gamma$ is a geodesic. Let us also recall that the scalar curvature of $\Gamma$ is

$$
\kappa^{s}=\left|\gamma^{\prime \prime}\right|=\left(\kappa_{g}^{2}+\kappa_{n}^{2}\right)^{1 / 2} .
$$

\subsection{Local coordinates near a curve inside the boundary}

Let $\Gamma$ be a curve in $\partial \Omega$ parametrized by arc lengths on some interval $I=\left[-a_{1},+a_{2}\right]$ : $\Gamma=\{\gamma(s) ; s \in I\}$. So we have $\left|\gamma^{\prime}(s)\right|=1$. Then, there exists a neighborhood $\mathcal{W}_{x_{0}}$ of $x_{0}=\gamma(0)$ in $\partial \Omega$, such that, for any $z \in \mathcal{W}_{x_{0}} \cap \Gamma$, there exists a unique geodesic $\Lambda_{z}$ through $z$ and normal to $\Gamma$. The neighborhood $\mathcal{W}_{x_{0}}$ of $x_{0}$ can also be choosen such that

$$
\forall x \in \mathcal{W}_{x_{0}}, \quad \exists ! z=z(x) \in \Gamma \cap \mathcal{W}_{x_{0}} \text { s.t. } d_{\partial \Omega}(x, z)=d_{\partial \Omega}(x, \Gamma),
$$

where $d_{\partial \Omega}(.,$.$) denotes the distance on \partial \Omega$.

Then, there exists an open set $S$ of $\mathbb{R}^{2}$ and a regular diffeomorphism

$$
\phi: \mathcal{W}_{x_{0}} \rightarrow S, \phi(x)=(r, s) \quad \text { with } \pm r=d_{\partial \Omega}(x, \Gamma)=d_{\partial \Omega}(x, \gamma(s)) .
$$

We observe that:

$$
x(0, s)=\gamma(s) .
$$

We choose a positive orientation (and this determines the choice of the sign of $r$ ), by imposing:

$$
\frac{\partial x}{\partial r}(0, s) \wedge \frac{\partial x}{\partial s}(0, s)=N(\gamma(s))
$$

where $N(x)$ is the interior normal of $\partial \Omega$ at the point $x \in \partial \Omega$. Then $(r, s)$ are local coordinates in $\mathcal{W}_{x_{0}}$ and observing that, for any fixed $s, r \mapsto x(r, s)$ is a parametrization by arc lengths of the geodesic $\Lambda_{\gamma(s)}$, we have

$$
\left|\frac{\partial x}{\partial r}(r, s)\right|=1
$$

and

$$
\left\langle\frac{\partial x}{\partial r}(0, s) \mid \frac{\partial x}{\partial s}(0, s)\right\rangle=0 .
$$

More precisely we have the following lemma.

\footnotetext{
${ }^{5}$ The definition of the geodesic curvature is not uniform in the literature. Some authors [7] use the opposite sign.
} 
LEMMA 8.2. - In the above local coordinates, the metric $G$ on $\partial \Omega$ is diagonal:

$$
G=d r \otimes d r+\alpha(r, s) d s \otimes d s
$$

On the curve $\Gamma$, we have

$$
\alpha(0, s)=1, \quad \frac{\partial \alpha}{\partial r}(0, s)=-2 \kappa_{g}(\gamma(s)) \quad \text { and } \quad \frac{\partial \alpha}{\partial s}(0, s)=0
$$

where $\kappa_{g}(\gamma(s))$ denotes the geodesic curvature of the curve $\Gamma$ at $\gamma(s)$.

Proof. - As for any fixed $s$, the map $r \mapsto x(r, s)$ is a parametrization by arc lengths of the geodesic $\Lambda_{\gamma(s)}$, the curvature of $\Lambda_{\gamma(s)}$ is given, using (8.11), (8.12) and Remark 8.1, by

$$
\frac{\partial^{2} x}{\partial r^{2}}(r, s)=K\left(\frac{\partial x}{\partial r}(r, s), \frac{\partial x}{\partial r}(r, s)\right) N(x(r, s)) .
$$

Then, we get from (8.20) that

$$
\frac{\partial}{\partial r}\left\langle\frac{\partial x}{\partial s} \mid \frac{\partial x}{\partial r}\right\rangle=\left\langle\frac{\partial^{2} x}{\partial s \partial r} \mid \frac{\partial x}{\partial r}\right\rangle=\frac{1}{2} \frac{\partial}{\partial s}\left\langle\frac{\partial x}{\partial r} \mid \frac{\partial x}{\partial r}\right\rangle .
$$

So, using in addition (8.16), we have

$$
\frac{\partial}{\partial r}\left\langle\frac{\partial x}{\partial s} \mid \frac{\partial x}{\partial r}\right\rangle=0
$$

But $\Lambda_{\gamma(s)}$ is normal to $\Gamma$; so $\left\langle\frac{\partial x}{\partial s}(0, s) \mid \frac{\partial x}{\partial r}(0, s)\right\rangle=0$. Then, using (8.22), we get:

$$
\left\langle\frac{\partial x}{\partial s}(r, s) \mid \frac{\partial x}{\partial r}(r, s)\right\rangle=0
$$

This shows that the metric is diagonal as announced in (8.18) with

$$
\alpha(r, s)=\left\langle\frac{\partial x}{\partial s}(r, s) \mid \frac{\partial x}{\partial s}(r, s)\right\rangle .
$$

We get also from the orthogonality of $\gamma^{\prime}$ and $\gamma^{\prime \prime}$ that

$$
\frac{\partial \alpha}{\partial s}(0, s)=0 .
$$

But, differentiating the identity (8.23) with respect to $s$, we have

$$
\frac{\partial \alpha}{\partial r}(r, s)=2\left\langle\frac{\partial^{2} x}{\partial s \partial r} \mid \frac{\partial x}{\partial s}\right\rangle=-2\left\langle\frac{\partial x}{\partial r} \mid \frac{\partial^{2} x}{\partial s^{2}}\right\rangle .
$$

But, by (8.11), we have:

$$
\frac{\partial^{2} x}{\partial s^{2}}(0, s)=\kappa_{n}(\gamma(s)) N(\gamma(s))+\kappa_{g}(\gamma(s)) \frac{\partial x}{\partial s}(0, s) \wedge N(\gamma(s)),
$$


so (8.15) and (8.17) lead to

$$
\frac{\partial^{2} x}{\partial s^{2}}(0, s)=\kappa_{n}(\gamma(s)) N(\gamma(s))+\kappa_{g}(\gamma(s)) \frac{\partial x}{\partial r}(0, s) .
$$

The formulas (8.24) and (8.25) give the non-obvious part of (8.19)

$$
\frac{\partial \alpha}{\partial r}(0, s)=-2 \kappa_{g}(\gamma(s))
$$

Remark 8.3. - In the coordinates $(r, s)$ introduced in Lemma 8.2, the second fundamental form is given (see (8.7)) by:

$$
K=K_{11} d r \otimes d r+K_{12} d r \otimes d s+K_{21} d s \otimes d r+K_{22} d s \otimes d s
$$

with:

$$
\begin{aligned}
& K_{11}(r, s)=\left\langle\frac{\partial^{2} x}{\partial r^{2}}(r, s) \mid N(x(r, s))\right\rangle, \\
& K_{22}(r, s)=\left\langle\frac{\partial^{2} x}{\partial s^{2}}(r, s) \mid N(x(r, s))\right\rangle, \\
& K_{12}(r, s)=\left\langle\frac{\partial^{2} x}{\partial r \partial s}(r, s) \mid N(x(r, s))\right\rangle, \\
& K_{21}(r, s)=K_{12}(r, s) .
\end{aligned}
$$

The function $K_{11}(r, s)$ is the normal curvature of the geodesic $\Lambda_{\gamma(s)}$ at $x(r, s)$ and the function $K_{22}(0, s)=\kappa_{n}(\gamma(s))$ is the normal curvature of the curve $\Gamma$ at $x(0, s)=\gamma(s)$.

\subsection{Local coordinates near a curve in the boundary}

We come back to previous computations and relate them to the curvatures. Let $\phi(x)=\left(y_{1}, y_{2}\right)$ be local coordinates of the boundary as defined in (8.1). We have observed in (5.19), that

$$
g_{0}=d y_{3} \otimes d y_{3}+\sum_{1 \leqslant i, j \leqslant 2}\left[G_{i j}\left(y_{1}, y_{2}\right)-2 y_{3} K_{i j}\left(y_{1}, y_{2}\right)+y_{3}^{2} L_{i j}\right] d y_{i} \otimes d y_{j}
$$

where

- $G=\sum_{1 \leqslant i, j \leqslant 2} G_{i j} d y_{i} \otimes d y_{j}$,

- $K=\sum_{1 \leqslant i, j \leqslant 2} K_{i j} d y_{i} \otimes d y_{j}$,

- $L=\sum_{1 \leqslant i, j \leqslant 2} L_{i j} d y_{i} \otimes d y_{j}=\sum_{1 \leqslant i, j \leqslant 2}\left\langle\frac{\partial N}{\partial y_{i}} \mid \frac{\partial N}{\partial y_{j}}\right\rangle d y_{i} \otimes d y_{j}$.

$G, K$ and $L$ are respectively called the first, second and third fundamental forms on $\partial \Omega$. If we take local coordinates $\left(y_{1}, y_{2}\right)=(r, s)$ on the boundary given by Lemma 8.2, the sesquilinear form introduced in (5.15) becomes

$$
q_{A}^{h}(u)=\int_{\mathcal{V}_{x_{0}}}|g|^{1 / 2}\left[\left|h D_{y_{3}}-\widetilde{A}_{3} u\right|^{2}+\sum_{1 \leqslant i, j \leqslant 2} g^{i j}\left(h D_{y_{i}} u-\widetilde{A}_{i} u\right) \cdot \overline{\left(h D_{y_{j}} u-\widetilde{A}_{j} u\right)}\right] d y^{3},
$$

for $u$ supported in $\mathcal{V}_{x_{0}}$, the associated differential operator is 


$$
\begin{aligned}
P_{A}^{h}= & \left(h D_{y_{3}}-\widetilde{A}_{3}\right)^{2}+\frac{h}{2 i}|g|^{-1}\left(\frac{\partial}{\partial y_{3}}|g|\right)\left(h D_{y_{3}}-\widetilde{A}_{3}\right) \\
& +|g|^{-1 / 2} \sum_{1 \leqslant i, j \leqslant 2}\left(h D_{y_{j}}-\widetilde{A}_{j}\right)\left(|g|^{1 / 2} g^{i j}\left(h D_{y_{i}}-\widetilde{A}_{i}\right)\right) .
\end{aligned}
$$

If we now consider the coordinates introduced in Section 8.2, that is $\left(y_{1}, y_{2}\right)=(s, t)$ and complete by $t=y_{3}$ introduced in Section 5.3, then

$$
|g|=\alpha(r, s)-2 t\left[\alpha(r, s) K_{11}(r, s)+K_{22}(r, s)\right]+t^{2} \varepsilon_{3}(r, s, t),
$$

and, for $1 \leqslant i, j \leqslant 2$,

$$
\left(g^{i j}\right)_{1 \leqslant i, j \leqslant 2}=\left(\begin{array}{cc}
1 & 0 \\
0 & \alpha^{-1}
\end{array}\right)+2 t\left(\begin{array}{cc}
K_{11} & \alpha^{-1} K_{12} \\
\alpha^{-1} K_{21} & \alpha^{-2} K_{22}
\end{array}\right)+t^{2} R
$$

where $\varepsilon_{3}$ and $R_{i j}$ are smooth functions.

\subsection{More magnetic geometry: discussion around our invariants}

We assume that the magnetic field $H=\operatorname{curl} A$ is constant and we can assume, without loss of generality, that:

$$
A(x)=\frac{b}{2}\left(0,-x_{3}, x_{2}\right)
$$

for some fixed $b>0$.

Let $\Omega$ be bounded open set of $\mathbb{R}^{3}$ with regular boundary $\partial \Omega$. We now assume that (1.5) is satisfied. We observe that this assumption is satisfied when $\Omega$ is strictly convex. We consider a parametrization $s \mapsto \gamma(s)$ of $\Gamma_{H}$ by arc length, and

$$
\theta(s)=\operatorname{Arcsin}\left(\left\langle\gamma^{\prime}(s) \mid \frac{H(B)}{|H(B)|}\right\rangle\right) .
$$

We have already introduced $\kappa_{n, B}$ in (1.6). Similarly, we can define:

$$
\kappa_{t, B}(\gamma(s))=K\left(\gamma^{\prime}(s), \frac{H(B)}{|H(B)|}\right)
$$

We observe that we have:

$$
\begin{aligned}
& \kappa_{n, B}(\gamma(s))=K\left(\frac{\partial}{\partial r}, \frac{H(B)}{|H(B)|}\right)=\cos \theta(s) K_{11}(0, s)+\sin \theta(s) K_{12}(0, s) ; \\
& \kappa_{t, B}(\gamma(s))=K\left(\frac{\partial}{\partial s}, \frac{H(B)}{|H(B)|}\right)=\cos \theta(s) K_{12}(0, s)+\sin \theta(s) K_{22}(0, s) .
\end{aligned}
$$

Let us observe that the angle $\theta(s)$ is not "free" in our picture. In fact we have the geometrical fact:

Proposition 8.4. - The assumption $H(B)$ is constant and tangent to the surface $\partial \Omega$ along the curve $\Gamma_{H}$ implies that

$$
\kappa_{t, B}(x)=0, \quad \forall x \in \Gamma_{H} .
$$

$4^{\mathrm{e}}$ SÉRIE - TOME $37-2004-\mathrm{N}^{\circ} 1$ 
Moreover, we have:

$$
\theta^{\prime}(s)=\kappa_{g}(\gamma(s)), \quad \forall s
$$

Proof. - Let us write:

$$
H(B)=\sin (\theta(s)) \gamma^{\prime}(s)+\cos (\theta(s))\left[\gamma^{\prime}(s) \wedge N(\gamma(s)]\right.
$$

As $H(B)$ is constant, we can differentiate with respect to $s(8.37)$ and we get:

$$
\begin{aligned}
& \theta^{\prime}(s)\left[\cos (\theta(s)) \gamma^{\prime}(s)-\sin (\theta(s)) \gamma^{\prime}(s) \wedge N(\gamma(s))\right]+\sin (\theta(s)) \gamma^{\prime \prime}(s) \\
& \quad+\cos (\theta(s))\left[\gamma^{\prime \prime}(s) \wedge N(\gamma(s))+\gamma^{\prime}(s) \wedge(N \circ \gamma)^{\prime}(s)\right]=0 .
\end{aligned}
$$

But using the coordinates of Lemma 8.2, formula (8.6) gives

$$
(N \circ \gamma)^{\prime}=-\left(K_{12} \circ \gamma\right) \gamma^{\prime} \wedge N(\gamma)-\left(K_{22} \circ \gamma\right) \gamma^{\prime},
$$

and (8.25) becomes

$$
\gamma^{\prime \prime}=\left(\kappa_{g} \circ \gamma\right) \gamma^{\prime} \wedge(N \circ \gamma)+\left(\kappa_{n} \circ \gamma\right)(N \circ \gamma)
$$

So

$$
\begin{aligned}
& \theta^{\prime}\left[\cos \theta \gamma^{\prime}-\sin \theta \gamma^{\prime} \wedge(N \circ \gamma)\right]+\sin \theta\left[\left(\kappa_{g} \circ \gamma\right) \gamma^{\prime} \wedge(N \circ \gamma)+\left(\kappa_{n} \circ \gamma\right)(N \circ \gamma)\right] \\
& \quad+\cos \theta\left[-\left(\kappa_{g} \circ \gamma\right) \gamma^{\prime}+\left(K_{12} \circ \gamma\right)(N \circ \gamma)\right]=0,
\end{aligned}
$$

and then, expressing the previous equality on the basis $\gamma^{\prime} \wedge(N \circ \gamma), \gamma^{\prime}, N \circ \gamma$,

$$
\begin{aligned}
& \cos \theta\left[\theta^{\prime}-\left(\kappa_{g} \circ \gamma\right)\right]=0, \\
& \sin \theta\left[-\theta^{\prime}+\left(\kappa_{g} \circ \gamma\right)\right]=0, \\
& \sin \theta\left(\kappa_{n} \circ \gamma\right)+\cos \theta\left(K_{12} \circ \gamma\right)=0 .
\end{aligned}
$$

We get (8.36), and using (8.12) we get also (8.35) from $\kappa_{n} \circ \gamma=K_{22} \circ \gamma$ and from (8.34).

Remark 8.5. - In the case when $\partial \Omega$ is strictly convex, $(K>0)$, then (8.35) implies that

$$
\kappa_{n, B} \neq 0, \quad \forall x \in \Gamma_{H}
$$

One can also meet degenerate cases where $K$ is not invertible (locally cylindric domains).

When $\theta(s)=0$, we deduce from (8.34) and (8.35) that $K_{12}(x(s))=0$. So the curvature matrix $K$ becomes diagonal.

Proof. - We observe that (8.34) can be rewritten at a point $x=\gamma(s)$ in the form:

$$
\left(\begin{array}{c}
\kappa_{n, B}(x) \\
\kappa_{t, B}(x)
\end{array}\right)=K_{x}\left(\begin{array}{c}
\cos \theta(s) \\
\sin \theta(s)
\end{array}\right) .
$$

Observing that $K_{x}$ is inversible when $\Omega$ is strictly convex ( $K_{x}$ is actually strictly positive), we immediately see that $\left|\kappa_{n, B}(x)\right|+\left|\kappa_{t, B}(x)\right| \neq 0$.

Remark 8.6. - The condition that $\theta^{\prime}=\kappa_{g} \circ \gamma$ is obtained also in the case $\theta \equiv 0$. So in this case we have effectively $\kappa_{g}=0$. So $\Gamma_{H}$ should be a geodesic. Conversely, when $\Gamma_{H}$ is a geodesic, 
we should have $\theta \equiv 0$ if $H(B)$ is constant. We observe indeed that by computing the circulation of $H(B)$ along $\Gamma_{H}$ and using Stokes Lemma (or more simply that $\left\langle H(B) \mid \gamma^{\prime}\right\rangle=\frac{d}{d s}\langle H(B) \mid \gamma\rangle$ ), we should have:

$$
\int_{a}^{b} \sin \theta(s) d s=0 .
$$

When $\theta$ is constant, this implies $\theta=0$.

Example 8.7. - Let us consider the case of the ellipsoid:

$$
a_{1} x_{1}^{2}+a_{2} x_{2}^{2}+a_{3} x_{3}^{2}=1 \text {. }
$$

It is interesting to compute our invariants. Take for simplification, the case when $B=(0,0,1)$. Then $\Gamma_{H}$ is the intersection of the ellipsoid with $x_{3}=0$. So we get an ellipse in this plane. We can now observe that the vector field $H$ is orthogonal to $\Gamma_{H}$. We observe that:

$$
\langle H \mid N\rangle=-|B| \frac{a_{3} x_{3}}{\nu},
$$

with

$$
\nu=\sqrt{a_{1}^{2} y_{1}^{2}+a_{2}^{2} y_{2}^{2}+a_{3}^{2} x_{3}^{2}} .
$$

This leads to:

$$
\left|\kappa_{n, B}\left(y_{1}, y_{2}\right)\right|=\frac{a_{3}}{\nu} .
$$

The minimum of $\kappa_{n, B}$ (which appears in formula (1.10)) is then obtained at the point where $\nu$ is maximal. If we assume for example that $a_{1}>a_{2}$, we get that this maximum is obtained at $x_{2}=x_{3}=0$ and equal to $a_{1}$. This differs from the intuition we got from the two-dimensional case.

Remark 8.8. - Using (8.32), we observe that we have:

$$
\sin ^{2}(\theta(x))=\left\langle T(x) \mid \frac{H(B)}{|H(B)|}\right\rangle^{2},
$$

and this permits to compare various formulations of the constant $\hat{\gamma}_{0}$ introduced in (1.10).

Remark 8.9. - The expression in (1.10) can be related more directly to the curvature by using the following formula:

$$
\begin{aligned}
\kappa_{n, B}(x)^{2}\left(\frac{1}{2} \mu^{\prime \prime}\left(\xi_{0}\right) \sin ^{2} \theta(x)+\cos ^{2} \theta(x)\right) \\
=\kappa^{G}(x)^{2}\left(\kappa_{n}(x)^{2}+\frac{1}{2} \mu^{\prime \prime}\left(\xi_{0}\right) K_{x}(T(x), T(x) \wedge N(x))\right) \\
\quad \times\left(\kappa_{n}(x)^{2}+K_{x}(T(x), T(x) \wedge N(x))\right)^{-2} .
\end{aligned}
$$

The proof is left to the reader. The interest of this formula is that its computation depends only on invariants related to $\Gamma_{H}$. 
Remark 8.10. - If $\kappa^{G} \neq 0$, then $\kappa_{n, B}$ is different from 0 . We have indeed:

$$
\kappa_{n, B}=\frac{\kappa^{G}}{\kappa_{n}} \cos \theta
$$

Note that if we assume in addition that

$$
\kappa_{n}(x) \neq 0,
$$

then $H$ can not be tangent to $\Gamma_{H}$. This would mean indeed that $\theta=\frac{\pi}{2}$, and by (8.34) and (8.35) that $K_{22}=0$.

\section{Towards the model: new normal forms}

In this section, the vector magnetic field $H(B)$, introduced in (5.3), is assumed to be constant, more precisely

$$
H(B)=b \frac{\partial}{\partial x_{1}}
$$

with $b=|H(B)|>0$. Moreover the set $\Gamma_{H}=\left\{x \in \partial \Omega ; H(B) \in T_{x}(\partial \Omega)\right\}$ is assumed to be a regular curve.

The angle between the normal to $\Gamma_{H}$ in $T_{x} \partial \Omega$ and $H(B)$ will be denoted by $\theta(x)$.

We will work near a point of $\Gamma_{H} x_{0}$ to be determined and will look for a good approximation of the operator in a small box around $x_{0}$.

We consider local coordinates $(r, s, t)$ in a neighborhood $\mathcal{V}_{x_{0}}$ of $x_{0}$, such that $(r, s)$ are the coordinates of Lemma 8.2, and $t$ is such that

$$
t=t(x)=\operatorname{distance}(x, \partial \Omega) .
$$

\subsection{Normal form for the magnetic potential}

In this subsection we shall show how after a suitable gauge transform, we can arrive to a more tractable model. We assume that the magnetic field is constant.

LEMma 9.1. - Let $\widetilde{A}$ be the magnetic potential defining $\widetilde{B}$, in the coordinates $(r, s, t)$ defined near $x_{0}=\left(0, s_{0}, 0\right)$. Let $\widetilde{A}^{(2)}$ be the Taylor expansion to order 2 of $\widetilde{A}$. Then there exists $a$ polynomial function of $y=\left(r, s-s_{0}, t\right), p^{0}(y)$ such that,

$$
\widetilde{A}^{(2)}=A^{0}+\operatorname{grad}_{y} p^{0},
$$

with

$$
\begin{aligned}
A_{1}^{0}= & b t\left[\sin \theta_{0}+\kappa_{g}\left(x_{0}\right) \cos \theta_{0}\left(s-s_{0}\right)-\sin \theta_{0} \kappa^{M}\left(x_{0}\right) t\right], \\
A_{2}^{0}= & -b t\left[\cos \theta_{0}-\kappa_{g}\left(x_{0}\right) \cos \theta_{0} r-\kappa_{g}\left(x_{0}\right) \sin \theta_{0}\left(s-s_{0}\right)-\cos \theta_{0} \kappa^{M}\left(x_{0}\right) t\right] \\
& -\frac{1}{2} b \kappa_{n, B}\left(x_{0}\right) r^{2}, \\
A_{3}^{0}= & 0 .
\end{aligned}
$$

Remark 9.2. - The proof will use assumption (1.8). We recall that this assumption is always satisfied in the strictly convex case. In this case $H(B)$ cannot be tangent to $\Gamma_{H}$. 
Proof. - To prove this lemma, we have just to determine the Taylor expansion up to order 1 of the magnetic field in the coordinates $\left(r, s-s_{0}, t\right)$ at the point $\left(0, s_{0}, 0\right)$. Let us first recall that we have assumed that $A$ was given by (8.31) and that the corresponding vector $H(B)$ is defined by (9.1). Let us analyze its expression in the new coordinates $(r, s, t)$ :

$$
H(B)=b \frac{\partial}{\partial x_{1}}=\tilde{b}_{1} \frac{\partial}{\partial r}+\tilde{b}_{2} \frac{\partial}{\partial s}+\tilde{b}_{3} \frac{\partial}{\partial t},
$$

and, by (8.26), (8.18) and (9.4), we get

$$
\begin{aligned}
& b \frac{\partial x_{1}}{\partial r}=\left\langle H(B) \mid \frac{\partial}{\partial r}\right\rangle=\tilde{b}_{1}\left(1-2 t K_{11}\right)-2 t \tilde{b}_{2} K_{12}+\mathcal{O}\left(t^{2}\right), \\
& b \frac{\partial x_{1}}{\partial s}=\left\langle H(B) \mid \frac{\partial}{\partial s}\right\rangle=\tilde{b}_{2}\left(\alpha-2 t K_{22}\right)-2 t \tilde{b}_{1} K_{12}+\mathcal{O}\left(t^{2}\right),
\end{aligned}
$$

and

$$
b \frac{\partial x_{1}}{\partial t}=\left\langle H(B) \mid \frac{\partial}{\partial t}\right\rangle=\tilde{b}_{3} .
$$

Using (8.19), (9.5), (9.6) and Schwarz Lemma for $x_{1}$, we get:

$$
\frac{\partial \tilde{b}_{1}}{\partial s}=\frac{\partial}{\partial r}\left(\alpha \tilde{b}_{2}\right)=-2\left(\kappa_{g} \circ \gamma\right) \tilde{b}_{2}+\frac{\partial \tilde{b}_{2}}{\partial r},
$$

when $t=r=0$.

We have:

$$
\tilde{b}_{1}=b \cos \theta(s), \quad \tilde{b}_{2}=b \sin \theta(s), \quad \tilde{b}_{3}=0,
$$

when $t=r=0$.

This leads by differentiation with respect to $s$ to:

$$
\begin{aligned}
& \frac{\partial \tilde{b}_{1}}{\partial s}=-b \sin \theta(s) \theta^{\prime}(s), \\
& \frac{\partial \tilde{b}_{2}}{\partial s}=b \cos \theta(s) \theta^{\prime}(s), \\
& \frac{\partial \tilde{b}_{3}}{\partial s}=0,
\end{aligned}
$$

when $t=r=0$. Then we get by coming back to (9.8) that (9.9) leads to:

$$
\frac{\partial \tilde{b}_{2}}{\partial r}=-b \sin \theta \theta^{\prime}+2\left(\kappa_{g} \circ \gamma\right) b \sin \theta
$$

Using (8.36), we get:

$$
\frac{\partial \tilde{b}_{2}}{\partial r}=\left(\kappa_{g} \circ \gamma\right) b \sin \theta
$$

We would like now to have an expression for $\frac{\partial \tilde{b}_{1}}{\partial r}$. We shall again use that $H(B)$ is a constant vector field and compute the square of its norm, using (5.14), formula (5.19) and Lemma 8.2. This leads to:

$$
b^{2}=\tilde{b}_{1}^{2}+\alpha(r, s) \tilde{b}_{2}^{2}+\tilde{b}_{3}^{2},
$$


on $t=0$.

Differentiating (9.13) with respect to $r$, we get for $r=t=0$,

$$
\tilde{b}_{1} \frac{\partial \tilde{b}_{1}}{\partial r}+\frac{1}{2} \frac{\partial \alpha}{\partial r}\left(\tilde{b}_{2}\right)^{2}+\tilde{b}_{2} \frac{\partial \tilde{b}_{2}}{\partial r}=0 .
$$

But we can now use (8.19) and (9.9) and we get:

$$
\cos \theta(s) \frac{\partial \tilde{b}_{1}}{\partial r}(0, s, 0)=0
$$

This gives finally, using (1.8):

$$
\frac{\partial \tilde{b}_{1}}{\partial r}=0,
$$

when $t=r=0$.

Let us now use (9.7) to write that

$$
\frac{\partial \tilde{b}_{3}}{\partial r}=\frac{\partial}{\partial r}\langle H(B) \mid N\rangle=\left\langle H(B) \mid \frac{\partial N}{\partial r}\right\rangle
$$

when $t=0$.

Then, using also (9.4), (9.9) and (8.6), we obtain:

$$
\left\langle H(B) \mid \frac{\partial N}{\partial r}\right\rangle=b \cos \theta(s)\left\langle\frac{\partial}{\partial r} \mid \frac{\partial N}{\partial r}\right\rangle+b \sin \theta(s)\left\langle\frac{\partial}{\partial s} \mid \frac{\partial N}{\partial r}\right\rangle .
$$

This leads to

$$
\frac{\partial \tilde{b}_{3}}{\partial r}=-b \cos \theta(s) K_{11}-b \sin \theta(s) K_{12}
$$

when $t=r=0$.

But by (9.5), (9.7) and Schwarz Lemma, we have

$$
\frac{\partial \tilde{b}_{3}}{\partial r}=\frac{\partial \tilde{b}_{1}}{\partial t}-2 \tilde{b}_{1} K_{11}-2 \tilde{b}_{2} K_{12}
$$

when $t=r=0$.

So, from (9.16), we get:

$$
\frac{\partial \tilde{b}_{1}}{\partial t}=b \cos \theta(s) K_{11}+b \sin \theta(s) K_{12},
$$

when $t=r=0$.

Once again, Schwarz Lemma, (9.6) and (9.7) lead to

$$
\frac{\partial \tilde{b}_{3}}{\partial s}=\alpha \frac{\partial \tilde{b}_{2}}{\partial t}-2 \tilde{b}_{2} K_{22}-2 \tilde{b}_{1} K_{12}
$$

when $t=0$. 
Then, (8.19), (9.9) and (9.10) lead to

$$
\frac{\partial \tilde{b}_{2}}{\partial t}=2 b \cos \theta K_{12}+2 b \sin \theta K_{22}
$$

when $t=r=0$.

It remains to determine $\frac{\partial \tilde{b}_{3}}{\partial t}$ when $t=r=0$. Let us denote the magnetic field

$$
\widetilde{B}=\widetilde{B}_{23} d s \wedge d t+\widetilde{B}_{31} d t \wedge d r+\widetilde{B}_{12} d r \wedge d s
$$

So by (5.11) and using the property that:

$$
\frac{\partial \widetilde{B}_{23}}{\partial r}+\frac{\partial \widetilde{B}_{31}}{\partial s}+\frac{\partial \widetilde{B}_{12}}{\partial t}=0,
$$

we get from (5.13) that

$$
|g|^{1 / 2}\left[\frac{\partial \tilde{b}_{1}}{\partial r}+\frac{\partial \tilde{b}_{2}}{\partial s}+\frac{\partial \tilde{b}_{3}}{\partial t}\right]+\frac{1}{2}|g|^{-1 / 2}\left[\left(\partial_{t}|g|\right) \tilde{b}_{3}+\left(\partial_{r}|g|\right) \tilde{b}_{1}+\left(\partial_{s}|g|\right) \tilde{b}_{2}\right]=0
$$

Let us now use this formula on $t=r=0$. Using (9.15), (9.10), we get first:

$$
\frac{\partial \tilde{b}_{3}}{\partial t}+b \cos \theta(s) \theta^{\prime}(s)=-\frac{1}{2}|g|^{-1}\left(\left(\partial_{t}|g|\right) \tilde{b}_{3}+\left(\partial_{r}|g|\right) \tilde{b}_{1}+\left(\partial_{s}|g|\right) \tilde{b}_{2}\right),
$$

on $t=r=0$.

We now use (8.29) and Lemma 8.2 (more precisely (8.19)), we obtain, using (9.9):

$$
\left(\partial_{r}|g|\right)=-2 \kappa_{g}(\gamma(s)), \quad \text { when } t=r=0 .
$$

This leads to:

$$
\frac{\partial \tilde{b}_{3}}{\partial t}=b\left(\kappa_{g} \circ \gamma-\theta^{\prime}\right) \cos \theta \quad \text { when } t=r=0 .
$$

We actually need a more complete expansion of $|g|$. We have:

$$
|g|=1-2 \kappa_{g}\left(\gamma\left(s_{0}\right)\right) r-2\left(K_{11}\left(0, s_{0}\right)+K_{22}\left(0, s_{0}\right)\right) t+\mathcal{O}\left(\left(t, r, s-s_{0}\right)^{2}\right) .
$$

We can establish the Taylor expansion of the magnetic field in the coordinates $(r, s, t)$.

We can now establish the formulas in full generality. For $t=r=0$, we have, with $\theta=\theta(s)$ :

$$
\begin{aligned}
& \tilde{b}_{1}=b \cos \theta, \\
& \frac{\partial \tilde{b}_{1}}{\partial r}=0, \\
& \frac{\partial \tilde{b}_{1}}{\partial s}=-b \sin \theta, \\
& \frac{\partial \tilde{b}_{1}}{\partial t}=b \cos \theta K_{11}+b \sin \theta K_{12} ;
\end{aligned}
$$


(9.26)

$$
\begin{aligned}
& \tilde{b}_{2}=b \sin \theta(s), \\
& \frac{\partial \tilde{b}_{2}}{\partial r}=b\left(2 \kappa_{g} \circ \gamma-\theta^{\prime}\right) \sin \theta, \\
& \frac{\partial \tilde{b}_{2}}{\partial s}=b \cos \theta \theta^{\prime}, \\
& \frac{\partial \tilde{b}_{2}}{\partial t}=2 b \cos \theta K_{12}+2 b \sin \theta K_{22} ; \\
& \frac{\tilde{b}_{3}=0,}{\frac{\partial \tilde{b}_{3}}{\partial r}=-b \cos \theta K_{11}-b \sin \theta K_{12},} \\
& \frac{\partial \tilde{b}_{3}}{\partial s}=0, \\
& \frac{\partial \tilde{b}_{3}}{\partial t}=b \cos \theta\left(\kappa_{g} \circ \gamma-\theta^{\prime}\right) .
\end{aligned}
$$

Using the Taylor extension (to order 1) of $|g|^{1 / 2}$,

$$
|g|^{1 / 2}=1-\kappa_{g}\left(\gamma\left(s_{0}\right)\right) r-\left(K_{11}\left(0, s_{0}\right)+K_{22}\left(0, s_{0}\right)\right) t+\mathcal{O}\left(\left|\left(r, s-s_{0}, t\right)\right|^{2}\right),
$$

we obtain the model:

$$
\begin{aligned}
\tilde{b}_{1}^{0}= & b \cos \theta\left(s_{0}\right)-b \sin \theta\left(s_{0}\right) \theta^{\prime}\left(s_{0}\right)\left(s-s_{0}\right)+\left(b \cos \theta\left(s_{0}\right) K_{11}\left(0, s_{0}\right)\right. \\
& \left.+b \sin \theta\left(s_{0}\right) K_{22}\left(0, s_{0}\right)\right) t \\
\tilde{b}_{2}^{0}= & b \sin \theta\left(s_{0}\right)-b \cos \theta\left(s_{0}\right) \theta^{\prime}\left(s_{0}\right)\left(s-s_{0}\right)+b \sin \theta\left(2 \kappa_{g}\left(\gamma\left(s_{0}\right)\right)-\theta^{\prime}\left(s_{0}\right)\right) r \\
& +\left(2 b \cos \theta K_{12}+2 b \sin \theta\left(s_{0}\right) K_{22}\right) t \\
\tilde{b}_{3}^{0}= & -\left(b \cos \theta\left(s_{0}\right) K_{11}+b \sin \theta\left(s_{0}\right) K_{12}\right) r+b \cos \theta\left(s_{0}\right)\left(\kappa_{g}\left(\gamma\left(s_{0}\right)\right)-\theta^{\prime}\left(s_{0}\right)\right) t .
\end{aligned}
$$

These formulas lead to the introduction of the "model" corresponding magnetic field:

$$
\begin{aligned}
\widetilde{B}_{23}^{0}= & b \cos \theta_{0}-b \sin \theta_{0} \theta^{\prime}\left(s_{0}\right)\left(s-s_{0}\right)-b \cos \theta_{0} \kappa_{g}\left(\gamma\left(s_{0}\right)\right) r \\
& +b\left(\left(\cos \theta_{0}-1\right) K_{11}+\left(\sin \theta_{0}-1\right) K_{22}\right) t, \\
\widetilde{B}_{31}^{0}= & b \sin \theta_{0}-b \cos \theta_{0} \theta^{\prime}\left(s_{0}\right)\left(s-s_{0}\right)+b \sin \theta_{0}\left(\kappa_{g}\left(\gamma\left(s_{0}\right)\right)-\theta^{\prime}\left(s_{0}\right)\right) r \\
& +b\left(2 \cos \theta_{0} K_{12}+\sin \theta_{0} K_{22}\right) t, \\
\widetilde{B}_{12}^{0}= & -b\left(\cos \theta_{0} K_{11}+\sin \theta_{0} K_{12}\right) r+b \cos \theta_{0}\left(\kappa_{g}\left(\gamma\left(s_{0}\right)\right)-\theta^{\prime}\left(s_{0}\right)\right) t,
\end{aligned}
$$

with $\theta_{0}=\theta\left(s_{0}\right), K_{i j}=K_{i j}\left(0, s_{0}\right)$.

This corresponds to the Taylor expansion up to order 1 of $\widetilde{B}$ at the point $\left(0, s_{0}, 0\right)$.

$$
\begin{aligned}
\tilde{b}_{1}= & b\left[\cos \left(\theta\left(s_{0}\right)\right)-\theta^{\prime}\left(s_{0}\right) \sin \left(\theta\left(s_{0}\right)\right)\left(s-s_{0}\right)+\kappa_{n, B}\left(x_{0}\right) t\right]+\mathcal{O}\left(|y|^{2}\right), \\
\tilde{b}_{2}= & b\left[\sin \left(\theta\left(s_{0}\right)\right)+\sin \left(\theta\left(s_{0}\right)\right)\left(2 \kappa_{g}\left(x_{0}\right)-\theta^{\prime}\left(s_{0}\right)\right) r\right. \\
& \left.+\theta^{\prime}\left(s_{0}\right) \cos \left(\theta\left(s_{0}\right)\right)\left(s-s_{0}\right)+2 \kappa_{t, B}\left(x_{0}\right) t\right]+\mathcal{O}\left(|y|^{2}\right), \\
\tilde{b}_{3}= & b\left[-\kappa_{n, B}\left(x_{0}\right) r+\left(\kappa_{g}\left(x_{0}\right)-\theta^{\prime}\left(s_{0}\right)\right) \cos \left(\theta\left(s_{0}\right)\right) t\right]+\mathcal{O}\left(|y|^{2}\right) .
\end{aligned}
$$


As from (8.29)

$$
|g|^{1 / 2}=1-\kappa_{g}\left(x_{0}\right) r-2 \kappa^{M} t+\mathcal{O}\left(|y|^{2}\right),
$$

we get using the relation between the magnetic field and the vector magnetic field given by (5.11) and (5.12)

$$
\begin{aligned}
\widetilde{B}_{23}= & b\left[\cos \theta_{0}-\kappa_{g}\left(x_{0}\right) \cos \theta_{0} r-\theta^{\prime}\left(s_{0}\right) \sin \theta_{0}\left(s-s_{0}\right)\right] \\
& +b\left(\kappa_{n, B}\left(x_{0}\right)-2 \cos \theta_{0} \kappa^{M}\left(x_{0}\right)\right) t+\mathcal{O}\left(|y|^{2}\right), \\
\widetilde{B}_{31}= & b\left[\sin \theta_{0}+\sin \theta_{0}\left(\kappa_{g}\left(x_{0}\right)-\theta^{\prime}\left(s_{0}\right)\right) r+\theta^{\prime}\left(s_{0}\right) \cos \theta_{0}\left(s-s_{0}\right)\right] \\
& +2 b t\left[\kappa_{t, B}\left(x_{0}\right)-\sin \theta_{0} \kappa^{M}\left(x_{0}\right)\right]+\mathcal{O}\left(|y|^{2}\right), \\
\widetilde{B}_{12}= & b\left[-\kappa_{n, B}\left(x_{0}\right) r+\left(\kappa_{g}\left(x_{0}\right)-\theta^{\prime}\left(s_{0}\right)\right) \cos \theta_{0} t\right]+\mathcal{O}\left(|y|^{2}\right),
\end{aligned}
$$

with $\theta_{0}:=\theta\left(s_{0}\right)$.

If we write that $|H(B)|=b$, using the relation $\theta^{\prime}\left(s_{0}\right)=\kappa_{g}\left(x_{0}\right)$, this leads first to:

$$
\begin{aligned}
& \tilde{b}_{1}=b\left[\cos \theta_{0}-\kappa_{g}\left(x_{0}\right) \sin \theta_{0}\left(s-s_{0}\right)+\kappa_{n, B}\left(x_{0}\right) t\right]+\mathcal{O}\left(|y|^{2}\right), \\
& \tilde{b}_{2}=b\left[\sin \theta_{0}+\sin \theta_{0} \kappa_{g}\left(x_{0}\right) r+\kappa_{g}\left(x_{0}\right) \cos \theta_{0}\left(s-s_{0}\right)\right]+\mathcal{O}\left(|y|^{2}\right), \\
& \tilde{b}_{3}=-b \kappa_{n, B}\left(x_{0}\right) r+\mathcal{O}\left(|y|^{2}\right),
\end{aligned}
$$

and then to:

$$
\begin{aligned}
\widetilde{B}_{23}= & b\left[\cos \theta_{0}-\kappa_{g}\left(x_{0}\right) \cos \theta_{0} r-\kappa_{g}\left(x_{0}\right) \sin \theta_{0}\left(s-s_{0}\right)\right. \\
& \left.+\left(\kappa_{n, B}\left(x_{0}\right)-2 \cos \theta_{0} \kappa^{M}\left(x_{0}\right)\right) t\right]+\mathcal{O}\left(|y|^{2}\right), \\
\widetilde{B}_{31}= & b\left[\sin \theta_{0}+\kappa_{g}\left(x_{0}\right) \cos \theta_{0}\left(s-s_{0}\right)-2 \sin \theta_{0} \kappa^{M}\left(x_{0}\right) t\right]+\mathcal{O}\left(|y|^{2}\right), \\
\widetilde{B}_{12}= & -b \kappa_{n, B}\left(x_{0}\right) r+\mathcal{O}\left(|y|^{2}\right) .
\end{aligned}
$$

By comparison of the curls on the left and right hand side of (9.2) we get the lemma.

\subsection{Towards simplified models}

If we neglect the terms of order $t^{2}$, we get the potential $A^{00}$ whose components are given by:

$$
A_{2}^{00}=-b t\left[\cos \theta_{0}-\kappa_{g}\left(x_{0}\right) \cos \theta_{0} r-\kappa_{g}\left(x_{0}\right) \sin \theta_{0}\left(s-s_{0}\right)\right]-b \frac{\kappa_{n, B}\left(x_{0}\right)}{2} r^{2},
$$

and

$$
A_{3}^{00}=0
$$

The corresponding magnetic field is:

$$
\begin{aligned}
& B_{23}^{00}=b \cos \theta_{0}-b \cos \theta_{0} \kappa_{g}\left(x_{0}\right) r-b \sin \theta_{0} \kappa_{g}\left(x_{0}\right)\left(s-s_{0}\right), \\
& B_{31}^{00}=b \sin \theta_{0}-b \cos \theta_{0} \kappa_{g}\left(x_{0}\right)\left(s-s_{0}\right), \\
& B_{12}^{00}=-b \kappa_{n, B}\left(x_{0}\right) r .
\end{aligned}
$$

If we neglect in addition the terms corresponding to $t\left(s-s_{0}\right)$, we get the magnetic potential 


$$
\begin{aligned}
& A_{1}^{01}=b t \sin \theta_{0}, \\
& A_{2}^{01}=-b t\left[\cos \theta_{0}-\kappa_{g}\left(x_{0}\right) \cos \theta_{0} r\right]-b \frac{\kappa_{n, B}\left(x_{0}\right)}{2} r^{2}, \\
& A_{3}^{01}=0 .
\end{aligned}
$$

The corresponding magnetic field is:

$$
\begin{aligned}
& B_{23}^{01}=b \cos \theta_{0}-b \cos \theta_{0} \kappa_{g}\left(x_{0}\right) r, \\
& B_{31}^{01}=b \sin \theta_{0}, \\
& B_{12}^{01}=-b \kappa_{n, B}\left(x_{0}\right) r .
\end{aligned}
$$

But we will start our analysis with the model, where we neglect also the terms in $\mathcal{O}(|r t|)$ :

$$
\begin{aligned}
& \hat{A}_{1}=b \sin \theta_{0} t, \\
& \hat{A}_{2}=-b \cos \theta_{0} t-\frac{b}{2} \kappa_{n, B}\left(s_{0}\right) r^{2}, \\
& \hat{A}_{3}=0 .
\end{aligned}
$$

Neglecting also the geodesic curvature, this leads to the model:

$$
P^{\text {mod }}:=\left(h D_{r}-\hat{A}_{1}\right)^{2}+\left(h D_{s}-\hat{A}_{2}\right)^{2}+h^{2} D_{t}^{2} .
$$

\section{Comparison lemmas near $\Gamma_{H}$}

Although the problem is easier in the case of upper bounds where we work with explicit quasimodes, we need in the two cases (proof of upper bounds and proof of lower bounds) comparison lemmas permitting to control the error made when considering the simplified models. Further comparison lemmas will be needed later.

\subsection{A first comparison lemma}

We are interested in the energy $q_{A}^{h}(u)$, introduced in (8.27), of some function $u$ in $H^{2}(\Omega)$ such that

$$
\operatorname{supp}(u) \subset Q\left(x_{0}\right),
$$

where

$$
Q\left(x_{0}\right)=\left\{x \in \Omega ;(r(x), s(x)) \in\left(r_{0}, s_{0}\right)+\left[-h^{\delta}, h^{\delta}\right]^{2}, t(x) \in\left[0, \varepsilon_{2}\right]\right\},
$$

for some $\delta \in] 0,1[$.

From now on, we assume in this section that $\left(r\left(x_{0}\right), s\left(x_{0}\right)\right)=\left(0, s_{0}\right)$.

LEMMA 10.1. - If (10.1) is satisfied, then

$$
\begin{aligned}
(1- & \left.C h^{2 \delta}\right) q_{\widetilde{A}^{(2)}}^{h}(u)-C\left\|t^{1 / 2}\left(h D_{x}-A\right) u\right\|^{2} \\
& \quad-C\left[q_{\widetilde{A}^{(2)}}^{h}(u)\right]^{1 / 2} \cdot\left\|\left(h^{3 \delta}+h^{2 \delta} t+h^{\delta} t^{2}+t^{3}\right) u\right\|-C\left\|\left(h^{3 \delta}+h^{2 \delta} t+h^{\delta} t^{2}+t^{3}\right) u\right\|^{2} \\
\leqslant & q_{A}^{h}(u) \\
\leqslant & \left(1+C h^{2 \delta}\right) q_{\widetilde{A}^{(2)}}^{h}(u)+C\left\|t^{1 / 2}\left(h D_{x}-A\right) u\right\|^{2}
\end{aligned}
$$




$$
\begin{aligned}
& +C\left[q_{\widetilde{A}^{(2)}}^{h}(u)\right]^{1 / 2} \cdot\left\|\left(h^{3 \delta}+h^{2 \delta} t+h^{\delta} t^{2}+t^{3}\right) u\right\| \\
& +C\left\|\left(h^{3 \delta}+h^{2 \delta} t+h^{\delta} t^{2}+t^{3}\right) u\right\|^{2}
\end{aligned}
$$

where:

$$
\begin{aligned}
q_{\widetilde{A}^{(2)}}^{h}(u)= & \int_{Q\left(x_{0}\right)}\left(1-r \kappa_{g}\left(x_{0}\right)\right)\left[\left|\left(h D_{t}-\widetilde{A}_{3}^{(2)}\right) u\right|^{2}\right. \\
& \left.+\left(1+2 r \kappa_{g}\left(x_{0}\right)\right)\left|\left(h D_{s}-\widetilde{A}_{2}^{(2)}\right) u\right|^{2}+\left|\left(h D_{r}-\widetilde{A}_{1}^{(2)}\right) u\right|^{2}\right] d r d s d t .
\end{aligned}
$$

$\widetilde{A}^{(2)}$ is the quadratic part of the Taylor expansion at $x_{0}$ of $\widetilde{A}$ defined in (8.27):

$$
\widetilde{A}^{(2)}(y)=\sum_{|\beta| \leqslant 2} \frac{\partial^{\beta} \widetilde{A}}{\partial y^{\beta}}\left(0, s_{0}, 0\right) \frac{y^{\beta}}{\beta !},
$$

with $y=\left(y_{1}, y_{2}, y_{3}\right)=\left(r, s-s_{0}, t\right)$.

The function $\kappa_{g}\left(x_{0}\right)$ is the geodesic curvature at $x_{0}$ introduced in (8.11).

We have actually two types of errors in order to control the comparison of $q_{A}^{h}(u)$ and $q_{\widetilde{A}^{(2)}}^{h}(u)$.

The first error is that we replace the initial metric in the coordinates $(r, s, t)$ by the new metric (see Lemma 8.2):

$$
g_{0}^{\text {new }}=d t \otimes d t+d r \otimes d r+\left(1-2 r \kappa_{g}\left(x_{0}\right)\right) d s \otimes d s
$$

with the corresponding approximation of $\left|g^{\text {new }}\right|^{1 / 2}$ by $\left(1-r \kappa_{g}\left(x_{0}\right)\right)$, and we consider a similar linearization (with respect to $r$ and $t$ ) for $g^{i j}$.

We then observe that, on the support of $u$, we have:

$$
\begin{gathered}
|g|^{1 / 2}=1-\kappa_{g}\left(x_{0}\right) r+\mathcal{O}(t)+\mathcal{O}\left(h^{2 \delta}\right), \\
g_{\text {new }}^{i j}-g^{i j}=\mathcal{O}(t)+\mathcal{O}\left(h^{2 \delta}\right) .
\end{gathered}
$$

The second error occurs when replacing $A$ by $\widetilde{A}^{(2)}$ and this leads to an error estimated by:

$$
A-\widetilde{A}^{(2)}=\mathcal{O}\left(|y|^{3}\right)=\mathcal{O}\left(\left(|t|+h^{\delta}\right)^{3}\right) .
$$

Once these estimates are satisfied, the lemma follows easily using the Cauchy-Schwarz inequality, after having written:

$$
\left(h D_{y}-A\right)=\left(h D_{y}-\widetilde{A}^{(2)}\right)+\left(A-\widetilde{A}^{(2)}\right) .
$$

\subsection{A second comparison lemma}

We now perform a gauge transform. We also decide to consider as remainder the term in $\mathcal{O}\left(t^{2}\right)$ in $A$. In addition we need to change of unknown in order to go back to the Lebesgue measure.

LEMMA 10.2. - If (10.1) is satisfied, then

$$
q_{A^{00}}^{h}(\tilde{u})-C\left\|t^{1 / 2}\left(h D_{x}-A\right) u\right\|^{2}
$$




$$
\begin{aligned}
& -C\left[q_{A^{00}}^{h}(\tilde{u})\right]^{1 / 2} \cdot\left\|\left(h^{3 \delta}+h+h^{2 \delta} t+t^{2}\right) u\right\| \\
& -C\left\|\left(h^{3 \delta}+h+h^{2 \delta} t+t^{2}\right) u\right\|^{2} \\
\leqslant & q_{A}^{h}(u) \\
\leqslant & q_{A^{00}}^{h}(\tilde{u})+C\left\|t^{1 / 2}\left(h D_{x}-A\right) u\right\|^{2} \\
& +C\left[q_{A}^{h}(\tilde{u})\right]^{1 / 2} \cdot\left\|\left(h^{3 \delta}+h+h^{2 \delta} t+t^{2}\right) u\right\| \\
& +C\left\|\left(h^{3 \delta}+h+h^{2 \delta} t+t^{2}\right) u\right\|^{2},
\end{aligned}
$$

where

$$
q_{A^{00}}^{h}(\tilde{u})=\int_{Q\left(x_{0}\right)}\left[\left|h D_{t} \tilde{u}\right|^{2}+\left(1+2 r \kappa_{g}\left(x_{0}\right)\right)\left|\left(h D_{s}-A_{2}^{00}\right) \tilde{u}\right|^{2}+\left|\left(h D_{r}-A_{1}^{00}\right) \tilde{u}\right|^{2}\right] d r d s d t,
$$

where $A^{00}$ was introduced in (9.34)-(9.36) and where $p^{0}$ is the polynomial function introduced in Lemma 9.1.

We recall that in the particular case when $\theta \equiv 0$, it results from (8.36) that:

$$
\kappa_{g}\left(x_{0}\right)=0
$$

for $x_{0} \in \Gamma_{H}$.

\section{Spectral theory for a simple model}

\subsection{Heuristics}

We would like to understand the following simplified model considered in (9.41):

$$
P_{0}:=\left(h D_{r}-\sin \theta t\right)^{2}+\left(h D_{s}+\cos \theta t+\kappa \frac{r^{2}}{2}\right)^{2}+h^{2} D_{t}^{2}
$$

on $\mathbb{R}^{2} \times \mathbb{R}^{+}$. Here $\theta$ is assumed to be fixed and $\kappa$ is a real parameter. We have moreover assumed for simplicity that

$$
b=1
$$

We will take later

$$
\kappa=\kappa_{n, B}\left(s_{0}\right), \quad \theta=\theta\left(s_{0}\right),
$$

but it is better to keep them as an independent parameter for the first part of the analysis.

We are interested in the analysis of the bottom of the spectrum but will concentrate on the research of $L^{2}$ normalized solutions $u^{h}$ such that: $\left\langle P_{0} u^{h}, u^{h}\right\rangle$ is minimal.

Scaling. We first introduce the following scaling: $t=h^{1 / 2} \tilde{t}, r=h^{1 / 3} \tilde{r}$ and the coefficients of the operator being independent of $s$ we take a Fourier transform in $s$. 
Dividing by $h$, we get that $P_{0} / h$ is unitary equivalent to:

$$
P_{1}:=\left(h^{1 / 6} D_{\tilde{r}}-\sin \theta \tilde{t}\right)^{2}+\left(h^{1 / 2} \sigma+\cos \theta \tilde{t}+\kappa h^{1 / 6} \frac{\tilde{r}^{2}}{2}\right)^{2}+D_{\tilde{t}}^{2}
$$

on $\mathbb{R}^{2} \times \mathbb{R}^{+}$. We can rewrite the operator in the form:

$$
\begin{aligned}
P_{1}= & D_{\tilde{t}}^{2}+\left(\tilde{t}-h^{1 / 6}\left[\sin \theta D_{\tilde{r}}-\cos \theta\left(h^{1 / 3} \sigma+\frac{\kappa}{2} \tilde{r}^{2}\right)\right]\right)^{2} \\
& +h^{1 / 6}\left(\cos \theta D_{\tilde{r}}+\sin \theta\left(h^{1 / 3} \sigma+\frac{\kappa}{2} \tilde{r}^{2}\right)\right)^{2} .
\end{aligned}
$$

We note that we have "formally" a lower bound of the type:

$$
P_{1} \geqslant P_{2},
$$

where:

$$
\begin{aligned}
P_{2}:= & \mu\left(h^{1 / 6} \sin \theta D_{\tilde{r}}-\cos \theta\left(h^{1 / 3} \sigma+\frac{\kappa}{2} \tilde{r}^{2}\right)\right) \\
& +\left(h^{1 / 6} \cos \theta D_{\tilde{r}}+\sin \theta\left(h^{1 / 3} \sigma+\frac{\kappa}{2} \tilde{r}^{2}\right)\right)^{2},
\end{aligned}
$$

now considered as an operator on $L^{2}\left(\mathbb{R}_{\tilde{r}, \sigma}^{2}\right)$. In order to find a suitable quasimode, it is better to first decompose it as an Hilbertian integral of operators $P_{2}(\sigma)$, this time defined on $L^{2}(\mathbb{R})$, and to look for a minimization on $\sigma$. In this context, it is natural to replace $\mu$ by its approximation at the bottom. We recover a differential model, which will give a good understanding of the problem, modulo an error term which has to be controlled.

We consequently analyze the family (depending on $\sigma$ )

$$
\begin{aligned}
P_{3}(\sigma):= & \Theta_{0}+\delta_{0}\left(h^{1 / 6}\left(\sin \theta D_{\tilde{r}}-\cos \theta\left(h^{1 / 3} \sigma+\frac{\kappa}{2} \tilde{r}^{2}\right)\right)-\xi_{0}\right)^{2} \\
& +h^{1 / 3}\left(\cos \theta D_{\tilde{r}}+\sin \theta\left(h^{1 / 3} \sigma+\frac{\kappa}{2} \tilde{r}^{2}\right)\right)^{2}
\end{aligned}
$$

with $\delta_{0}=\frac{1}{2} \mu^{\prime \prime}\left(\xi_{0}\right)$.

It is now better to introduce

$$
\begin{gathered}
\sigma(\theta)=-\xi_{0} \cos \theta, \rho(\theta)=-\xi_{0} \sin \theta, \\
\sigma=\sigma(\theta) h^{-1 / 2}+h^{-1 / 3} \hat{\sigma},
\end{gathered}
$$

which permits to rewrite $P_{3}(\sigma)$ in the form:

$$
\begin{aligned}
P_{3}(\sigma):= & \Theta_{0}+\delta_{0}\left(h^{1 / 6}\left(\sin \theta\left(D_{\tilde{r}}+\rho(\theta) h^{-1 / 6}\right)-\cos \theta\left(\hat{\sigma}+\frac{\kappa}{2} \tilde{r}^{2}\right)\right)\right)^{2} \\
& +h^{1 / 3}\left(\cos \theta\left(D_{\tilde{r}}+\rho(\theta) h^{-1 / 6}\right)+\sin \theta\left(\hat{\sigma}+\frac{\kappa}{2} \tilde{r}^{2}\right)\right)^{2} .
\end{aligned}
$$

A translation in $\hat{\sigma}$ and a gauge transform by $\exp i h^{-1 / 6} \rho(\theta) r$ leads then to the analysis of the family: 


$$
\begin{aligned}
P_{4}(\hat{\sigma}):= & \Theta_{0}+h^{1 / 3} \delta_{0}\left(\sin \theta D_{\tilde{r}}-\cos \theta\left(\hat{\sigma}+\frac{\kappa}{2} \tilde{r}^{2}\right)\right)^{2} \\
& +h^{1 / 3}\left(\cos \theta D_{\tilde{r}}+\sin \theta\left(\hat{\sigma}+\frac{\kappa}{2} \tilde{r}^{2}\right)\right)^{2}
\end{aligned}
$$

\subsection{Analysis of the simplified model}

It is then natural to introduce:

$$
P_{5}(\hat{\sigma}):=h^{-1 / 3}\left(P_{4}(\hat{\sigma})-\Theta_{0}\right)
$$

which becomes independent of $h$ :

$$
\begin{aligned}
P_{5}(\hat{\sigma})= & \delta_{0}\left(-\cos \theta\left(\frac{\kappa}{2} r^{2}+\hat{\sigma}\right)+\sin \theta D_{r}\right)^{2} \\
& +\left(\cos \theta D_{r}+\sin \theta\left(\frac{\kappa}{2} r^{2}+\hat{\sigma}\right)\right)^{2}
\end{aligned}
$$

Here we have omitted the tilde's for the next computations.

Our aim is to first minimize over $\hat{\sigma}$ and then to minimize over the points of $\Gamma_{H}$, remembering that $\kappa=\kappa_{n, B}(x)$ and $\theta=\theta(x)$ with $x$ in $\Gamma_{H}$.

Let us now show, that by a gauge transform, we can rewrite $P_{5}(\hat{\sigma})$ in the form:

$$
P_{6}(\hat{\sigma})=c D_{r}^{2}+d\left(r^{2}-\rho\right)^{2} .
$$

We look for a gauge transformation of the form:

$$
t(\theta, r)=\alpha(\theta)\left(\frac{\kappa}{6} r^{3}+\hat{\sigma r}\right) .
$$

We consider

$$
P_{6}(\hat{\sigma}):=\exp -i t(\theta, r) \cdot P_{5}(\hat{\sigma}) \cdot \exp i t(\theta, r) .
$$

The function $\alpha(\theta)$ in (11.14) is chosen such that the coefficients of the operator

$$
\left(\frac{\kappa}{2} r^{2}+\hat{\sigma}\right) D_{r}+D_{r}\left(\frac{\kappa}{2} r^{2}+\hat{\sigma}\right)
$$

vanish. This leads to:

$$
\alpha(\theta)=\frac{\sin \theta \cos \theta\left(1-\delta_{0}\right)}{\delta_{0} \sin ^{2} \theta+\cos ^{2} \theta} .
$$

Of course, we have

$$
c=\cos ^{2} \theta+\delta_{0} \sin ^{2} \theta
$$

and

$$
\rho=2 \hat{\sigma} / \kappa
$$


But for this value of $\alpha(\theta)$, we get:

$$
d=\left(\frac{\kappa}{2}\right)^{2}\left(\delta_{0}(\cos \theta+\alpha(\theta) \sin \theta)^{2}+(-\sin \theta+\alpha(\theta) \cos \theta)^{2}\right)
$$

After computation, this gives:

$$
d=\left(\delta_{0}\left(\frac{\kappa}{2}\right)^{2}\right) /\left(\delta_{0} \sin ^{2} \theta+\cos ^{2} \theta\right) .
$$

We now rescale the operator $c D_{r}^{2}+d\left(r^{2}-\rho\right)^{2}$. We recall that we deleted the tilde's for simplicity. But we come back to the former writing ${ }^{6}$ and consider:

$$
P_{6}(\hat{\sigma})=c D_{\tilde{r}}^{2}+d\left(\tilde{r}^{2}-\rho\right)^{2} .
$$

This means that we perform a new scaling:

$$
\tilde{r}=\left(\frac{c}{d}\right)^{1 / 6} r^{\prime}
$$

such that $P_{6}(\hat{\sigma})$ becomes in the new coordinates:

$$
P_{7}(\hat{\sigma})=d^{1 / 3} c^{2 / 3}\left(D_{r^{\prime}}^{2}+\left(\left(r^{\prime}\right)^{2}-\rho^{\prime}\right)^{2}\right)
$$

with

$$
\rho^{\prime}=\left(\frac{c}{d}\right)^{-1 / 3} \rho
$$

We observe that $c$ and $d$ are independent of $\hat{\sigma}$. So in order to minimize over $\sigma$ the bottom of the spectrum of the initial operator, we will have to minimize over $\rho^{\prime}$, the bottom of the spectrum of the operator $\left(D_{r^{\prime}}^{2}+\left(\left(r^{\prime}\right)^{2}-\rho^{\prime}\right)^{2}\right)$ which is obtained for $\rho^{\prime}=\rho_{\min }$ and take the value $\hat{\nu}_{0}$ introduced in (2.15). This corresponds to

$$
\hat{\sigma}=\frac{\kappa}{2}\left(\frac{c}{d}\right)^{1 / 3} \rho_{\min }
$$

with

$$
\frac{c}{d}=\left(\cos ^{2} \theta+\delta_{0} \sin ^{2} \theta\right)^{2}\left(\delta_{0}\left(\frac{\kappa}{2}\right)^{2}\right)^{-1} .
$$

So, the bottom of the spectrum of $P_{7}$, is given for this value of $\hat{\sigma}$ by:

$$
d^{1 / 3} c^{2 / 3} \hat{\nu}_{0}=\left(\frac{1}{2}\right)^{2 / 3} \delta_{0}^{1 / 3}|\kappa|^{2 / 3}\left(\delta_{0} \sin ^{2} \theta+\cos ^{2} \theta\right)^{1 / 3} \hat{\nu}_{0} .
$$

If we now remember the values of $\kappa$ and $\theta$ in our application, this leads to:

\footnotetext{
${ }^{6}$ This is better, if we want to follow all the scalings we have done in the construction of quasimodes. 
PROPOSITION 11.1. - The ground state energy of the model operator $P_{4}$ is given by:

$$
\inf _{\hat{\sigma}} \inf \operatorname{Sp} P_{4}(\hat{\sigma})=\Theta_{0}+h^{1 / 3} \hat{\nu}_{0}\left(\frac{1}{2}\right)^{2 / 3} \delta_{0}^{1 / 3}|\kappa|^{2 / 3}\left(\delta_{0} \sin ^{2} \theta+\cos ^{2} \theta\right)^{1 / 3}
$$

Moreover the minimum is obtained for $\hat{\sigma}$ defined in (11.21).

In our application $\kappa$ and $\theta$ are not independent but satisfy (11.2) for some $x$ in $\Gamma_{H}$. This suggests that we have to look for a minimum over $\Gamma_{H}$ of the expression:

$$
\kappa_{n, B}(x)^{2}\left(\delta_{0} \sin ^{2} \theta(x)+\cos ^{2} \theta(x)\right)
$$

that is:

$$
\ell^{2}:=\inf _{x \in \Gamma_{H}}\left(\left(\kappa_{n, B}(x)\right)^{2}\left(\delta_{0} \sin ^{2} \theta(x)+\cos ^{2} \theta(x)\right)\right) .
$$

Here we recall that we use in this subsection a slightly different notation: $\theta(x)$ was previously written $\theta(s)$ with $x=\gamma(s)$.

\section{Proof of Theorem 1.2: upper bounds in general}

We give now the rigorous proof of the upper bound. This is reformulated in the

PROPOSITION 12.1. - Let $P^{h, N}$ be the Neumann magnetic Laplace operator on $L^{2}(\Omega)$ $(h D-A)^{2}$, where $\Omega$ is a bounded open set of $\mathbb{R}^{3}$ with smooth boundary $\partial \Omega$. We assume that the magnetic field $H=\operatorname{curl} A$ is constant and that (1.5) and (1.7) are satisfied. Then there exist $\eta>0$ and $C_{0}>0$ such that, for all $\left.\left.h \in\right] 0,1\right]$,

$$
\inf \operatorname{Sp}\left(P^{h, N}\right) \leqslant h b \Theta_{0}+h^{4 / 3} b^{2 / 3} \hat{\gamma}_{0}+C_{0} h^{\frac{4}{3}+\eta},
$$

where $b=|H|$ and $\hat{\gamma}_{0}$ is defined in (1.10).

Proof. - This is based on the MiniMax principle. We use Lemma 10.2 and we have just to find an $L^{2}$ normalized function $\tilde{u}$, supported in $h^{\delta} Q$, with $\left.Q=\right]-C, C\left[{ }^{2} \times[0, C[\right.$, such that

$$
q_{A^{00}}^{h}(\tilde{u}):=\int_{h^{\delta} Q}\left[\left|\left(h D_{r}-A_{1}^{00}\right) \tilde{u}\right|^{2}+\left(1+2 \kappa_{g}^{0} r\right)\left|\left(h D_{s}-A_{2}^{00}\right) \tilde{u}\right|^{2}+h^{2}\left|D_{t} \tilde{u}\right|^{2}\right] d r d s d t
$$

satisfies

$$
q_{A^{00}}^{h}(\tilde{u}) \leqslant \Theta_{0} b h+b^{2 / 3} \hat{\nu}_{0}\left(\mu^{\prime \prime}\left(\xi_{0}\right)\right)^{1 / 3} \hat{\gamma}_{0}, h^{4 / 3}+C h^{\eta+\frac{4}{3}},
$$

for some $\delta \in] \frac{5}{18}, \frac{1}{3}[$ and some $\eta>0$, and

$$
h^{-1}\left\|\left(1+h^{-1 / 4} t^{1 / 2}\right)\left(h D_{y}-\widetilde{A}\right) u\right\|^{2}+\left\|\left(1+h^{-1 / 2} t\right)^{2} u\right\|^{2} \leqslant C,
$$

with $u$ related to $\tilde{u}$ through (10.6),

$$
\begin{aligned}
& A_{1}^{00}(r, s, t)=b t\left[\sin \theta_{0}+\kappa_{g}^{0} \cos \theta_{0} s\right], \\
& A_{2}^{00}(r, s, t)=b t\left[-\cos \theta_{0}+\kappa_{g}^{0} \cos \theta_{0} r+\kappa_{g}^{0} \sin \theta_{0} s\right]-\frac{b}{2} \kappa_{n, B}^{0} r^{2},
\end{aligned}
$$




$$
\begin{aligned}
& \theta_{0}=\theta\left(x_{0}\right)=\operatorname{Arcsin}\left(\left|\left\langle T\left(x_{0}\right) \mid \frac{H}{b}\right\rangle\right|\right), \\
& \kappa_{g}^{0}=\kappa_{g}\left(x_{0}\right), \\
& \kappa_{n, B}^{0}=\kappa_{n, B}\left(x_{0}\right),
\end{aligned}
$$

and where $x_{0} \in \Gamma_{H}$ is chosen such that

$$
\tilde{\gamma_{0}}\left(x_{0}\right)=\hat{\gamma}_{0},
$$

where we recall that $\tilde{\gamma}_{0}$ was defined in (1.9). We have chosen a system of coordinates $(r, s, t)$ such that: $x_{0}=(0,0,0)$. We recall that $A^{00}$ was introduced in (9.34)-(9.36).

We search for a function of the form

$$
\begin{aligned}
\tilde{u}(r, s, t ; h)= & c_{1} h^{-\delta / 2} \exp \left(-\frac{i}{h^{1 / 3}} \rho \kappa_{n, B}^{0} s\right) \\
& \times \exp \left(\frac{i}{h^{1 / 2}}\left(\sin \theta_{0} r-\cos \theta_{0} s\right) b^{1 / 2} \xi_{0}\right) \cdot \chi\left(h^{-\delta} C^{-1} s\right) v(r, t),
\end{aligned}
$$

where $c_{1}$ is a normalization constant, $\rho$ is a constant to be determined later, $\chi$ is an even function on $\mathbb{R}$ supported on $\left[-\frac{1}{2}, \frac{1}{2}\right]$ and equal to 1 on $\left[-\frac{1}{4}, \frac{1}{4}\right]$.

As $s \mapsto \chi^{h}(s):=c h^{-\delta / 2} \chi\left(h^{-\delta} C^{-1} s\right)$ is an even function and

$$
\left\|\left(h D_{s}-b \kappa_{g}^{0} \sin \theta_{0} s t\right) \chi^{h}\right\|_{L^{2}\left(\mathbb{R}_{s}\right)}^{2} \leqslant C\left(h^{2-2 \delta}+h^{2 \delta} t^{2}\right),
$$

we only have to search for a normalized function $v(r, t)$ satisfying

$$
q_{M^{00}}^{h}(v) \leqslant h b \Theta_{0}+h^{4 / 3} b^{2 / 3} \hat{\nu}_{0}\left(\mu^{\prime \prime}\left(\xi_{0}\right)\right)^{1 / 3} \gamma_{0}+C h^{\eta+\frac{4}{3}},
$$

with

$$
q_{M^{00}}^{h}(v):=\int_{h^{\delta} Q_{2}}\left[\left|\left(h D_{r}-M_{1}^{00}\right) v\right|^{2}+\left|M_{2}^{00} v\right|^{2}+h^{2}\left|D_{t} v\right|^{2}\right] d r d t,
$$

$\left.Q_{2}=\right]-C, C[\times[0, C[$, and

$$
\begin{aligned}
M_{1}^{00}(r, t)= & b^{1 / 2} \sin \theta_{0}\left(b^{1 / 2} t-h^{1 / 2} \xi_{0}\right), \\
M_{2}^{00}(r, t)= & \left(1+2 \kappa_{g}^{0} r\right)^{1 / 2}\left[-b^{1 / 2} \cos \theta_{0}\left(b^{1 / 2} t-h^{1 / 2} \xi_{0}\right)+\kappa_{g}^{0} \cos \theta_{0} b r t\right. \\
& \left.-\frac{b}{2} \kappa_{n, B}^{0}\left(r^{2}-h^{2 / 3} b^{-1} \rho\right)\right] .
\end{aligned}
$$

Moreover $v$ must satisfy

(12.11) $h^{-1} \int_{h^{\delta} Q_{2}}\left(1+h^{-1 / 2} t\right)\left[\left|\left(h D_{r}-M_{1}^{00}\right) v\right|^{2}+\left|M_{2}^{00} v\right|^{2}+h^{2}\left|D_{t} v\right|^{2}\right] d r d t \leqslant C$,

and

$$
\left\|\left(1+h^{-1 / 2} t\right)^{2} v\right\|^{2} \leqslant C
$$

$4^{\mathrm{e}}$ SÉRIE - TOME $37-2004-\mathrm{N}^{\circ} 1$ 
So we can neglect the $t r^{2}$ and $r^{3}$ terms in $M_{2}^{00}$ which will lead to error terms in (12.8), and we get the new

$$
\begin{aligned}
M_{1}^{01}(r, t):= & b^{1 / 2} \sin \theta_{0}\left(b^{1 / 2} t-h^{1 / 2} \xi_{0}\right) \\
M_{2}^{01}(r, t):= & -b^{1 / 2} \cos \theta_{0}\left(b^{1 / 2} t-h^{1 / 2} \xi_{0}\right)+h^{1 / 2} b^{1 / 2} \xi_{0} \kappa_{g}^{0} \cos \theta_{0} r \\
& -\frac{b}{2} \kappa_{n, B}^{0}\left(r^{2}-h^{2 / 3} b^{-1} \rho\right) .
\end{aligned}
$$

We introduce the scaling $b^{1 / 2}\left(h^{-1 / 3} r, h^{-1 / 2} t\right)=(\hat{r}, \hat{t})$ and take $v$ in the form:

$$
v(r, t)=b^{1 / 2} h^{-5 / 12} v_{0}(\hat{r}, \hat{t}) .
$$

We then delete the hats and we get easily that

$$
q_{M^{01}}^{h}(v)=b h \tilde{q}^{h}\left(v_{0}\right)
$$

with

$$
\begin{aligned}
& \tilde{q}^{h}\left(v_{0}\right):=\int_{Q^{h}}\left[\left|D_{t} v_{0}\right|^{2}+\left|\left(t-\xi_{0}-h^{1 / 6} L_{1}^{h}\left(r, D_{r}\right)\right) v_{0}\right|^{2}+h^{1 / 3}\left|L_{2}^{h}\left(r, D_{r}\right) v_{0}\right|^{2}\right] d r d t \\
& \left.Q^{h}=\right]-C b^{1 / 2} h^{\delta-\frac{1}{3}}, C b^{1 / 2} h^{\delta-\frac{1}{3}}\left[\times\left[0, h^{\delta-\frac{1}{2}} b^{1 / 2}[\right.\right.
\end{aligned}
$$

and with

$$
\begin{aligned}
& L_{1}^{h}\left(r, D_{r}\right)=\sin \theta_{0} D_{r}-\cos \theta_{0} \frac{b^{-1 / 2}}{2}\left[\kappa_{n, B}^{0}\left(r^{2}-\rho\right)-2 h^{1 / 6} \xi_{0} \kappa_{g}^{0} \cos \theta_{0} r\right] \\
& L_{2}^{h}\left(r, D_{r}\right)=\cos \theta_{0} D_{r}+\sin \theta_{0} \frac{b^{-1 / 2}}{2}\left[\kappa_{n, B}^{0}\left(r^{2}-\rho\right)-2 h^{1 / 6} \xi_{0} \kappa_{g}^{0} \cos \theta_{0} r\right]
\end{aligned}
$$

We search for a function $v_{0}$ such that

$$
\left\|\left(1+r^{2}\right) v_{0}\right\| \leqslant C\left\|v_{0}\right\|
$$

We can neglect terms with $h^{1 / 6}$ in factor, and the $L_{j}^{h}$ are replaced by:

$$
\begin{aligned}
& L_{1}^{0}\left(r, D_{r}\right)=\sin \theta_{0} D_{r}-\cos \theta_{0} \frac{b^{-1 / 2}}{2}\left[\kappa_{n, B}^{0}\left(r^{2}-\rho\right)\right] \\
& L_{2}^{0}\left(r, D_{r}\right)=\cos \theta_{0} D_{r}+\sin \theta_{0} \frac{b^{-1 / 2}}{2}\left[\kappa_{n, B}^{0}\left(r^{2}-\rho\right)\right] .
\end{aligned}
$$

Let $\varphi_{0}(t)$ be the normalized eigenfunction of $Q\left(\xi_{0}\right)$ associated to the groundstate energy $\Theta_{0}$ (cf. Section 2.2). Then one can check easily as in [12] that there exists $C_{e}$ such that

$$
\left\|Q(\xi) \phi^{\xi}-\left[\Theta_{0}+\frac{1}{2}\left(\xi-\xi_{0}\right)^{2} \mu^{\prime \prime}\left(\xi_{0}\right)\right] \phi^{\xi}\right\| \leqslant C_{e}\left|\xi-\xi_{0}\right|^{3},
$$

where

$$
\phi^{\xi}(t)=\varphi_{0}(t)+\left(\xi-\xi_{0}\right) \varphi_{1}(t)+\left(\xi-\xi_{0}\right)^{2} \varphi_{2}(t)
$$




$$
\varphi_{1}=2\left[Q\left(\xi_{0}\right)-\Theta_{0}\right]^{-1, r}\left[\left(t-\xi_{0}\right) \varphi_{0}\right]
$$

and

$$
\varphi_{2}=2\left[Q\left(\xi_{0}\right)-\Theta_{0}\right]^{-1, r}\left[\left(t-\xi_{0}\right) \varphi_{1}-\left\langle\left(t-\xi_{0}\right) \varphi_{1} \mid \varphi_{0}\right\rangle \varphi_{0}\right]
$$

Here $\left(Q\left(\xi_{0}\right)-\Theta_{0}\right)^{-1, r}$ is the regularized resolvent defined by:

$$
\begin{aligned}
& \left(Q\left(\xi_{0}\right)-\Theta_{0}\right)^{-1, r} \varphi_{0}=0, \\
& \left(Q\left(\xi_{0}\right)-\Theta_{0}\right)^{-1, r} u=\left(Q\left(\xi_{0}\right)-\Theta_{0}\right)^{-1} u \quad \text { if }\left\langle u \mid \varphi_{0}\right\rangle=0 .
\end{aligned}
$$

The inequality (12.18) is first proved for $\tau=\xi-\xi_{0}$ small enough by perturbation, but we then claim that it is also true for $|\tau|>\tau_{0}>0$, if we observe that the square of the left hand side of (12.18) is a polynomial of degree 4 with respect to the $\tau$ variable.

By (12.18) and using Fourier transform, we know that there exist functions $\varphi_{j}(t)$ $(j=0, \ldots, 2)$, which are exponentially decreasing at infinity on $[0,+\infty[$ with their derivatives, such that, for any $\psi \in \mathcal{S}(\mathbb{R})$, if

$$
w^{h}(r, t)=\varphi_{0}(t) \psi(r)+\varphi_{1}(t) h^{1 / 6} L_{1}^{0}\left(r, D_{r}\right) \psi(r)+\varphi_{2}(t) h^{1 / 3}\left(L_{1}^{0}\left(r, D_{r}\right)\right)^{2} \psi(r),
$$

then we get,

$$
\begin{aligned}
& \left\|\left[D_{t}^{2}+\left(t-\xi_{0}-h^{1 / 6} L_{1}^{0}\left(r, D_{r}\right)\right)^{2}-\left(\Theta_{0}+\frac{1}{2} \mu^{\prime \prime}\left(\xi_{0}\right) h^{1 / 3}\left(L_{1}^{0}\left(r, D_{r}\right)\right)^{2}\right)\right] w^{h}\right\| \\
& \quad \leqslant C_{e} h^{1 / 2}\left\|\left(L_{1}^{0}\left(r, D_{r}\right)\right)^{3} w^{h}\right\| .
\end{aligned}
$$

This is indeed a consequence of the estimate (12.18) and of a suitable functional calculus. For defining this functional calculus, we can, for example, when $\theta_{0} \neq 0$, use a gauge transform which transforms $L_{1}^{0}$ into $\sin \theta_{0} D_{r}$ and the proof can then be done by partial Fourier transform. In the case when $\theta_{0}=0$ a direct proof can be done. Note that the constant $C_{e}$ is independent of $\theta_{0}$.

Let us define

$$
M\left(r, D_{r}\right):=\frac{1}{2} \mu^{\prime \prime}\left(\xi_{0}\right) L_{1}^{0}\left(r, D_{r}\right)^{2}+L_{2}^{0}\left(r, D_{r}\right)^{2} .
$$

At this point, we have justified the heuristic part of the previous section and can use the results obtained for the model (11.12) in Section 11.2. Let $\psi_{0}$ be the normalized eigenfunction (cf. Section 2.4) associated to the groundstate energy $\hat{\nu}_{0}$ (cf. (2.15)) of the Hamiltonian $D_{r}^{2}+\left(r^{2}-\rho_{\text {min }}\right)^{2}$ on $L^{2}(\mathbb{R})$. We recall that $r \mapsto \psi_{0}(r)$ is an even function.

Let

$$
\psi^{0}(r)=\left(\frac{c}{d}\right)^{-1 / 12} \exp i \varphi(r) \psi_{0}\left(\left(\frac{c}{d}\right)^{-1 / 6} r\right),
$$

where $\varphi(r)$ corresponds (modulo the scaling leading to $b=1$ ) to the gauge transform introduced in (11.14), and

$$
\psi=\psi^{0} \cdot \chi\left(C^{-1} h^{-\delta+\frac{1}{3}} r\right) .
$$

We take (see (12.7)):

$$
\rho=b^{1 / 3}\left(\frac{c}{d}\right)^{1 / 3} \rho_{\min },
$$

with $c$ and $d$ as in (11.22), $\gamma=\kappa_{n, B}^{0}$ and $\theta=\theta_{0}$. 
We then consider, with $w^{h}(r, t)$ defined in (12.22) and $\psi$ therein defined in (12.26),

$$
v_{0}(r, t)=w^{h}(r, t) \chi\left(\frac{1}{C} h^{-\delta+\frac{1}{2}} t\right) .
$$

We recall that $\psi_{0}$ is exponentially decreasing at infinity with all its derivatives.

Then we get easily from (12.23) and (11.24) that

$$
\tilde{q}_{A^{00}}^{h}\left(v_{0}\right) \leqslant\left[\Theta_{0}+h^{1 / 3} \hat{\gamma}_{0} b^{-1 / 3}+C h^{1 / 2}\right] \cdot\left\|v_{0}\right\|^{2} .
$$

Remark 12.2. - It is possible to treat more degenerate situations, for example if there exists $x_{0} \in \partial \Omega$ such that the Gauss curvature at $x_{0}$ vanishes, $H$ is tangent to $\partial \Omega$ at $x_{0}$ and the normal curvature at $x_{0}$ along the vector $H$ vanishes:

$$
H \in T_{x_{0}} \partial \Omega, \quad \kappa^{G}\left(x_{0}\right)=0, \quad K_{x_{0}}(H, H)=0 .
$$

We refer to [15] for a result in this direction.

\section{New lower bounds for generic models}

\subsection{Introduction}

We come back to the model analyzed in Section 11. We recall that we were interested in the model:

$$
P_{0}=\left(h D_{r}-\sin \theta t\right)^{2}+\left(h D_{s}+\cos \theta t+\frac{\kappa}{2} r^{2}\right)^{2}+h^{2} D_{t}^{2} .
$$

It is useful to consider the dilation $t=h^{1 / 2} \hat{t}, r=h^{1 / 3} \hat{r}$ and to divide the operator by $h$.

This leads to:

$$
\hat{P}_{0}=\left(h^{1 / 6} D_{\hat{r}}-\sin \theta \hat{t}\right)^{2}+\left(h^{1 / 2} D_{s}+\cos \theta \hat{t}+\frac{\kappa}{2} h^{1 / 6} \hat{r}^{2}\right)^{2}+D_{\hat{t}}^{2} .
$$

We look at functions supported in $\hat{I}(h) \times \mathbb{R} \times \overline{\mathbb{R}^{+}}$, where

$$
\hat{I}(h)=]-C h^{\delta-\frac{1}{3}}, C h^{\delta-\frac{1}{3}}[.
$$

Here $\delta \in] \frac{1}{4}, \frac{1}{3}[$, but other restrictions will appear during the proof.

After a new dilation and a partial Fourier transform with respect to the variable $s$, the starting point is the operator:

$$
\begin{aligned}
P(h, \sigma, \theta):= & D_{t}^{2}+\left(t-h^{1 / 6}\left[\sin \theta D_{r}-\cos \theta\left(\sigma+\frac{\kappa}{2} r^{2}\right)\right]\right)^{2} \\
& +h^{1 / 3}\left(\cos \theta D_{r}+\sin \theta\left(\sigma+\frac{\kappa}{2} r^{2}\right)\right)^{2}
\end{aligned}
$$

that we would like to analyze in $\hat{I}(h) \times \mathbb{R} \times \mathbb{R}_{+}$. We choose to consider first the problem for a family of operators depending on a parameter $\sigma$ and we would like to have a uniform lower 
bound (with respect to $\sigma$, of $\langle P(h, \sigma, \theta) u, u\rangle$ for $u$ 's supported in $\{(r, t) \mid r \in \hat{I}(h), t \geqslant 0\}$. It is convenient to rewrite $P(h, \sigma, \theta)$ in the form:

$$
P(h, \sigma, \theta)=D_{t}^{2}+\left(t-\xi_{0}-h^{1 / 6} L_{1}\right)^{2}+h^{1 / 3} L_{2}^{2},
$$

Here $L_{1}$ and $L_{2}$ are the differential operators of order 1 defined by

$$
\begin{aligned}
& L_{1}:=\sin \theta D_{r}-\cos \theta\left(\sigma+\frac{\kappa}{2} r^{2}\right)-\xi_{0} h^{-1 / 6}, \\
& L_{2}:=\cos \theta D_{r}+\sin \theta\left(\sigma+\frac{\kappa}{2} r^{2}\right) .
\end{aligned}
$$

Remark 13.1. - We have subtracted $\xi_{0} h^{-1 / 6}$ for simplicity. Note that because everything should be uniform with respect to $\sigma$, this is not really important, because the bracket of $L_{1}$ and $L_{2}$ is unchanged. A gauge transform by $\exp i\left(\xi_{0} \sin \theta h^{-1 / 6} r\right)$ gives indeed:

$$
\begin{aligned}
& L_{1}^{\text {new }}:=\sin \theta D_{r}-\cos \theta\left(\sigma+\frac{\kappa}{2} r^{2}+\xi_{0} h^{-1 / 6} \cos \theta\right), \\
& L_{2}^{\text {new }}:=\cos \theta D_{r}+\sin \theta\left(\sigma+\frac{\kappa}{2} r^{2}+\xi_{0} h^{-1 / 6} \cos \theta\right) .
\end{aligned}
$$

\subsection{Proof of the lower bound}

We will have to treat two cases: $\theta=0$ and $\left.\theta \in] 0, \frac{\pi}{2}\right]$. Because we can recover the first case from the case $\theta \neq 0$ by a continuity argument (see [14] for a direct proof) we always treat first the case $\left.\theta \in] 0, \frac{\pi}{2}\right]$ but we control the constants uniformly with respect to $\theta$.

The operator we would like to study has the following structure.

$$
P:=\mu\left(h^{1 / 6} L_{1}+\xi_{0}\right)-\Theta_{0}+h^{1 / 3} L_{2}^{2} .
$$

What follows is based on a variant of the functional calculus of two non commuting operators $L_{1}$ and $L_{2}$. This is related to operators appearing in the representation theory of rank 3 nilpotent groups. We observe indeed that all the brackets of order 4 vanish (see Helffer-Nourrigat [16]).

Remark 13.2. - For the justification of some abstract formulas, it could be useful to use a gauge transformation (which is singular for $\theta=0$ ), and leading to

$$
\begin{aligned}
& \hat{L}_{1}:=\sin \theta D_{r}, \\
& \hat{L}_{2}:=\cos \theta D_{r}+\frac{1}{\sin \theta}\left(\sigma+\xi_{0} h^{-1 / 6} \cos \theta+\frac{\kappa}{2} r^{2}\right)
\end{aligned}
$$

but it is better, at least at the beginning, to remain "abstract" noting that for any bounded $\chi$ one can associate by a functional calculus a bounded operator $\chi\left(L_{1}\right)$ defined on $L^{2}$.

A partition of unity. Let $\chi_{1}, \chi_{2}$ on $\mathbb{R}$, with $\chi_{1}$ equal to 1 in a neighborhood of the origin, say on $]-1,+1[$ and with support in $]-2,+2$ [, that we dilate by some factor $h^{\tau}$ :

$$
\chi_{j, h}(\rho)=\chi_{j}\left(h^{\tau} \rho\right) .
$$


We keep of course the property:

$$
\chi_{1, h}^{2}+\chi_{2, h}^{2}=1
$$

and we can obtain the following commutator formula:

$$
L_{2} \chi_{j, h}\left(L_{1}\right) u=\chi_{j, h}\left(L_{1}\right) L_{2} u+i h^{\tau} \kappa \chi_{j}^{\prime}\left(h^{\tau} L_{1}\right) r+h^{2 \tau} \frac{\kappa}{2} \sin (\theta) \chi_{j}^{\prime \prime}\left(h^{\tau} L_{1}\right) .
$$

We first write, with $P$ defined in (13.8):

$$
\begin{aligned}
\langle P u, u\rangle= & \left\langle P \chi_{1, h}\left(L_{1}\right) u, \chi_{1, h}\left(L_{1}\right) u\right\rangle+\left\langle P \chi_{2, h}\left(L_{1}\right) u, \chi_{2, h}\left(L_{1}\right) u\right\rangle \\
& -h^{2 \tau+\frac{1}{3}} \kappa \sin (\theta) \operatorname{Re}\left\{\sum _ { j } \left[\left\langle\chi_{j}^{\prime \prime}\left(h^{\tau} L_{1}\right) u, \chi_{j, h}\left(L_{1}\right) L_{2} u\right\rangle\right.\right. \\
& \left.\left.-i h^{\tau} \kappa\left\langle\chi_{j}^{\prime \prime}\left(h^{\tau} L_{1}\right) u, \chi_{j, h}^{\prime \prime}\left(L_{1}\right) r u\right\rangle\right]\right\} \\
& -h^{2 \tau+\frac{1}{3}} \frac{\kappa^{2}}{4} \sum_{j}\left[\left\|\chi_{j}^{\prime}\left(h^{\tau} L_{1}\right) r u\right\|^{2}+h^{2 \tau} \sin ^{2}(\theta)\left\|\chi_{j}^{\prime \prime}\left(h^{\tau} L_{1}\right) u\right\|^{2}\right] .
\end{aligned}
$$

We have used here that $\sum_{j=1}^{2} \chi_{j, h}^{\prime} \chi_{j, h}=0$.

Step 1 . We first observe that, by the properties of $\mu$ and estimating from below the second term of $P$, in (13.8) by 0 , we have, if $\tau \in] 0, \frac{1}{6}[$ :

$$
\left\langle P \chi_{2, h}\left(L_{1}\right) u, \chi_{2, h}\left(L_{1}\right) u\right\rangle \geqslant \frac{1}{C} h^{\frac{1}{3}-2 \tau}\left\|\chi_{2, h}\left(L_{1}\right) u\right\|^{2}, \quad \forall u \in \mathcal{S}(\mathbb{R}) .
$$

Everything is uniform, with respect to $\left.\theta \in] 0, \frac{\pi}{2}\right]$ and is valid for $h$ small enough. We get that, for any $C^{\prime}$, there exists $h_{0}\left(C^{\prime}\right)$ such that, $\left.\left.\forall h \in\right] 0, h_{0}\left(C^{\prime}\right)\right]$,

$$
\left\langle P \chi_{2, h}\left(L_{1}\right) u, \chi_{2, h}\left(L_{1}\right) u\right\rangle \geqslant C^{\prime} h^{1 / 3}\left\|\chi_{2, h}\left(L_{1}\right) u\right\|^{2} .
$$

Step 2. We consider now the first term. Using now the Taylor expansion of $\mu$ at $\xi_{0}$, we get:

$$
\begin{aligned}
\left\langle P \chi_{1, h}\left(L_{1}\right) u, \chi_{1, h}\left(L_{1}\right) u\right\rangle \geqslant & h^{1 / 3}\left(\delta_{0}\left\|L_{1} \chi_{1, h}\left(L_{1}\right) u\right\|^{2}+\left\|L_{2} \chi_{1, h}\left(L_{1}\right) u\right\|^{2}\right) \\
& -C h^{1 / 2}\left\langle\left|L_{1}\right|^{3} \chi_{1, h}\left(L_{1}\right) u, \chi_{1, h}\left(L_{1}\right) u\right\rangle .
\end{aligned}
$$

This is first transformed into:

$$
\begin{aligned}
\left\langle P \chi_{1, h}\left(L_{1}\right) u, \chi_{1, h}\left(L_{1}\right) u\right\rangle \geqslant & h^{1 / 3}\left(\delta_{0}\left\|L_{1} \chi_{1, h}\left(L_{1}\right) u\right\|^{2}+\left\|L_{2} \chi_{1, h}\left(L_{1}\right) u\right\|^{2}\right) \\
& -C h^{\frac{1}{2}-3 \tau}\left\|\chi_{1, h}\left(L_{1}\right) u\right\|^{2} .
\end{aligned}
$$

The analysis of this term is then reduced to the analysis of the simplified model:

$$
\delta_{0} L_{1}^{2}+L_{2}^{2}
$$

which is done in Section 11.2 and we also have to observe that a good remainder in (13.14) is obtained if $\frac{1}{2}-3 \tau>\frac{1}{3}$ :

$$
\tau \in] 0, \frac{1}{18}[
$$


Again, we emphasize that $C$ is independent of $h, \theta, \kappa$ and of $\sigma$.

$$
\begin{aligned}
& \left\langle P \chi_{1, h}\left(L_{1}\right) u, \chi_{1, h}\left(L_{1}\right) u\right\rangle \\
& \quad \geqslant h^{1 / 3} c^{\operatorname{conj}}(\kappa, \theta)\left\|\chi_{1, h}\left(L_{1}\right) u\right\|^{2}-C h^{\frac{1}{2}-3 \tau}\left\|\chi_{1, h}\left(L_{1}\right) u\right\|^{2},
\end{aligned}
$$

where

$$
c^{\operatorname{conj}}(\kappa, \theta):=\hat{\nu}_{0}\left(\frac{1}{2}\right)^{2 / 3} \delta_{0}^{1 / 3}(\kappa)^{2 / 3}\left(\delta_{0} \sin ^{2} \theta+\cos ^{2} \theta\right)^{1 / 3} .
$$

We now glue together the estimates (13.12), (13.13) and (13.16), then using that by (13.3) we have

$$
\forall u \in C_{0}^{\infty}(\hat{I}(h)), \quad\|r u\| \leqslant C h^{\delta-\frac{1}{3}}\|u\|,
$$

we get the following lemma.

LEMMA 13.3. - For any $\tau \in] 0, \frac{1}{18}[$, there exists a constant $C$, such that, for any $\theta$, for any $\sigma$, for any $u \in C_{0}^{\infty}(\hat{I}(h))$, we have

$$
\begin{aligned}
& \left\langle\left(h^{-1 / 3}\left(\mu\left(\xi_{0}+h^{1 / 6} L_{1}\right)-\Theta_{0}\right)+L_{2}^{2}\right) u, u\right\rangle \\
& \quad \geqslant c^{\operatorname{conj}}(\kappa, \theta)\|u\|^{2}-C h^{2\left(\tau+\delta-\frac{1}{3}\right)}\|u\|^{2}-C h^{\frac{1}{6}-3 \tau}\|u\|^{2}-C h^{2 \tau}\|u\| \cdot\left\|L_{2} u\right\| .
\end{aligned}
$$

Here $c^{\operatorname{conj}}(\kappa, \theta)$ is the constant introduced in (13.17).

It is clear that the above inequality implies:

$$
\begin{aligned}
& \left\langle\left(h^{-1 / 3}\left(\mu\left(\xi_{0}+h^{1 / 6} L_{1}\right)-\Theta_{0}\right)+L_{2}^{2}\right) u, u\right\rangle \\
& \quad \geqslant c^{\operatorname{conj}}(\kappa, \theta)\|u\|^{2}-C\left(h^{2\left(\tau+\delta-\frac{1}{3}\right)}+h^{\tau}+h^{\frac{1}{6}-3 \tau}\right)\|u\|^{2} .
\end{aligned}
$$

We also see that this is satisfactory if (13.19)

$$
\delta-\frac{1}{3}+\tau>0
$$

is satisfied.

Taking $\tau=\frac{1}{24}$ we conclude with the following lemma.

LEMMA 13.4. - For any $\delta \in] 0, \frac{1}{3}[$, there exists a constant $C$, such that, for any $\theta$, for any $\sigma$, for any $u \in C_{0}^{\infty}(]-C h^{\delta}, C h^{\delta}\left[\times \mathbb{R} \times \overline{\mathbb{R}^{+}}\right)$, we have

$$
\left\langle P_{0} u, u\right\rangle \geqslant\left[h \Theta_{0}+h^{4 / 3} c^{\text {conj }}-C\left(h^{11 / 8}+h^{\delta+\frac{13}{12}}\right)\right]\|u\|^{2} .
$$

Here we recall that $P_{0}$ is the operator defined in (13.1).

\section{Proof of Theorem 1.2: preliminaries for the lower bounds}

\subsection{Decomposition in four zones}

Before to enter into the technicalities, let us explain the ideas which lead to the partition of unity we shall consider.

We cut $\bar{\Omega}$ in different zones. 
- The first zone corresponds to the points which are far from the boundary. They are treated rather easily, exactly as in the case of dimension 2 . Here the model with constant magnetic field in $\mathbb{R}^{3}$ is relevant. This corresponds to the introduction of $\Gamma_{\tau(h)}^{0}$ already done in (7.16). The other zones correspond to a finer decomposition of $\Gamma_{\tau(h)}^{1}$ also already introduced in (7.16).

- The second zone $\Gamma_{\tau(h)}^{11}$ corresponds to the points which are near $\Gamma_{H}$ at a distance which is $\mathcal{O}\left(h^{\delta}\right)$. The model which was analyzed in the previous section is essentially relevant. This will be treated in Section 15.

- The third zone $\Gamma_{\tau(h)}^{13}$ corresponds to the points which are near $\partial \Omega$ but far from $\Gamma_{H}$. Here the model analyzed in Section 3 is relevant.

- Unfortunately, we need an intermediate zone. This will correspond to the introduction of $\Gamma_{\tau(h)}^{12}$ corresponding to points near $\Gamma_{H}$ but not in the second zone. In some sense, we need to interpolate between the second zone and the third zone. This will be treated in Section 16. Then, we have to glue together the estimates introduced in each zone and to control the errors related to the partition.

\subsection{A partition of unity}

We proceed as in the proof of Proposition 7.2. In the partition of unity (7.14), we take

$$
\left.\tau(h)=h^{\delta} \quad \text { with } \delta \in\right] \frac{5}{18}, \frac{1}{3}[.
$$

Let us denote by $u^{h}$ a normalized eigenfunction associated to the ground state energy $\lambda(h)$ of $P^{h, N}$. We recall that:

$$
\lambda(h)=q_{A}^{h}\left(u^{h}\right) \leqslant q_{A}^{h}(v) /\|v\|^{2}, \quad \forall v \in H^{1}(\Omega) .
$$

As announced in Section 14.1, we split $\Gamma_{\tau(h)}^{1}$, which was defined by (7.16), in the following way. For $C_{1}>1$ fixed large enough, let us define

$$
\begin{aligned}
& \Gamma_{\tau(h)}^{11}=\left\{\gamma \in \Gamma_{\tau(h)}^{1} ; \operatorname{dist}\left(\operatorname{supp}\left(\chi_{\gamma, \tau(h)}\right), \Gamma_{H}\right)<C_{1} \tau(h)\right\} \\
& \Gamma_{\tau(h)}^{12}=\left\{\gamma \in \Gamma_{\tau(h)}^{1} ; C_{1} \tau(h) \leqslant \operatorname{dist}\left(\operatorname{supp}\left(\chi_{\gamma, \tau(h)}\right), \Gamma_{H}\right)<C_{1}^{-1}\right\}, \\
& \Gamma_{\tau(h)}^{13}=\left\{\gamma \in \Gamma_{\tau(h)}^{1} ; C_{1}^{-1} \leqslant \operatorname{dist}\left(\operatorname{supp}\left(\chi_{\gamma, \tau(h)}\right), \Gamma_{H}\right)\right\} .
\end{aligned}
$$

\subsection{First zone: $\Gamma_{\tau(h)}^{0}$}

This was already analyzed in Section 7.3. We established in (7.18), that:

LEMMA 14.1.-

$$
\sum_{\gamma \in \Gamma_{\tau(h)}^{0}} q_{A}^{h}\left(\chi_{\gamma, \tau(h)} u^{h}\right) \geqslant h b \sum_{\gamma \in \Gamma_{\tau(h)}^{0}}\left\|\chi_{\gamma, \tau(h)} u\right\|^{2} .
$$

\subsection{Third zone: $\Gamma_{\tau(h)}^{13}$}

We use the proof of Proposition 7.2. We observe that we are in the first case considered in the proof of this proposition. We use (7.25) and get (7.27) in the following modified form: 


$$
\begin{gathered}
q_{A}^{h}\left(\chi_{\gamma, \tau(h)} u\right)+C(\tau(h))^{2}\left\|\chi_{\gamma, \tau(h)} u\right\| \cdot\left(q_{A}^{h}\left(\chi_{\gamma, \tau(h)} u\right)\right)^{1 / 2} \\
\geqslant\left[b h\left(\Theta_{0}+c_{3}\right)-C\left(h \tau(h)+(\tau(h))^{4}\right)\right] \cdot\left\|\chi_{\gamma, \tau(h)} u\right\|^{2},
\end{gathered}
$$

where $c_{3}>0$. This leads to the existence of $c_{4}>0$ and $h_{0}>0$ such that, for any $u \in H^{1}(\Omega)$ and any $\left.h \in] 0, h_{0}\right]$,

$$
q_{A}^{h}\left(\chi_{\gamma, \tau(h)} u\right) \geqslant h b\left(\Theta_{0}+c_{4}\right)\left\|\chi_{\gamma, \tau(h)} u\right\|^{2}, \quad \forall \gamma \in \Gamma_{\tau(h)}^{13}
$$

Taking $u=u^{h}$ and summing over $\gamma \in \Gamma_{\tau(h)}^{13}$, we get

LEMmA 14.2. - There exist $c_{3}>0$ and $h_{0}>0$ such that, for any $\left.\left.h \in\right] 0, h_{0}\right]$,

$$
\sum_{\gamma \in \Gamma_{\tau(h)}^{13}} q_{A}^{h}\left(\chi_{\gamma, \tau(h)} u^{h}\right) \geqslant h b\left(\Theta_{0}+c_{3}\right) \sum_{\gamma \in \Gamma_{\tau(h)}^{13}}\left\|\chi_{\gamma, \tau(h)} u\right\|^{2} .
$$

\section{Proof of Theorem 1.2: lower bounds in the second zone}

\subsection{A new comparison lemma}

We start from the conclusion (10.7) of Lemma 10.2. Omitting the tilda's, we consider the quadratic form $q_{m}^{h}$ (which was denoted by $q_{A^{\text {o0 }}}^{h}$ there) on $L^{2}\left(\Omega^{h}\right)$, where $\left.\Omega^{h}=Q^{h} \times\right] 0, h^{\delta}[$, with $\left.Q^{h}=\right]-h^{\delta}, h^{\delta}[\times]-h^{\delta}, h^{\delta}[$, which is defined by:

$$
q_{m}^{h}(u)=\int_{\Omega^{h}}\left[\left|h D_{r} u-A_{1} u\right|^{2}+\alpha(r)\left|h D_{s} u-A_{2} u\right|^{2}+h^{2}\left|D_{t} u\right|^{2}\right] d r d s d t,
$$

for all $u \in \mathcal{D}_{0}$, where

$$
\mathcal{D}_{0}=\left\{v \in H^{1}\left(\Omega^{h}\right), v /\left(\partial Q^{h}\right) \times\right] 0, h^{\delta}\left[=0, v / Q^{h} \times\left\{h^{\delta}\right\}=0\right\} .
$$

Here

$$
\begin{aligned}
& A_{1}=A_{1}(s, t)=b t a_{1}(s), \\
& A_{2}=A_{2}(r, s, t)=b t a_{2}(r, s)-\frac{b}{2} \kappa_{n, B} r^{2},
\end{aligned}
$$

with

$$
\begin{aligned}
& a_{1}(s)=\sin \theta+\kappa_{g} \cos \theta s, \\
& a_{2}(r, s)=-\cos \theta+\kappa_{g} \cos \theta r+\kappa_{g} \sin \theta s, \\
& \alpha(r)=1+2 \kappa_{g} r .
\end{aligned}
$$

Here

$$
\kappa_{g}=\kappa_{g}\left(x_{0}\right), \quad \theta=\theta\left(x_{0}\right) \quad \text { and } \quad \kappa_{n, B}=\kappa_{n, B}\left(x_{0}\right) .
$$

The coordinates of $x_{0}$ are $\left(0, s_{0}, 0\right)$ and we take in what follows $s_{0}=0$ (after a change of origin in the $s$-variable). As already mentioned these formulas coincide (modulo some change of notation) with the formulas (9.34)-(9.36). We assume that $b>0, \delta>0$ and that $\left.h \in] 0, h_{0}\right]$ with $h_{0}$ small enough. We assume also that $\alpha(r)>1 / 2$.

We denote by $P_{m}^{h}$ the associated self-adjoint operator on $L^{2}\left(\Omega^{h}\right)$. 
As before $C$ will denote some constant independent of $h$, which can change from a line to another.

Let us remark for future use that

$$
\left\|h D_{r} u\right\|^{2}+\left\|h D_{s} u\right\|^{2} \leqslant C\left[q_{m}^{h}(u)+\|t u\|^{2}+h^{4 \delta}\|u\|^{2}\right], \quad \forall u \in \mathcal{D}_{0} .
$$

LEMmA 15.1. - For any $\tau_{1}>0$, there exists $C>0$ such that, for any $u \in \mathcal{D}_{0}$,

$$
\left(1+C h^{2 \delta}\right) q_{m}^{h}(u) \geqslant\left(1-C h^{\tau_{1}}\right) q_{m, 0}^{h}(u)-C\left[\left(h^{2 \delta}+h^{\tau_{1}}\right)\|t u\|^{2}+h^{6 \delta-\tau_{1}}\|u\|^{2}\right]
$$

with

$$
q_{m, 0}^{h}(u)=\int_{\Omega^{h}}\left[h^{2}\left|D_{t} u\right|^{2}+\left|b t u-L_{0}^{h} u\right|^{2}+\left|L_{1}^{h} u\right|^{2}\right] d r d s d t
$$

and

$$
\begin{aligned}
L_{0}^{h} & =a_{1} h D_{r}+a_{2}^{0} h D_{s}-\frac{b}{2} \cos \theta \kappa_{n, B} r^{2}, \\
L_{1}^{h} & =a_{2}^{1} h D_{r}+a_{1}^{1} h D_{s}+\frac{b}{2} \sin \theta \kappa_{n, B} r^{2}, \\
a_{2}^{0} & =-\cos \theta-\kappa_{g} \cos \theta r+\kappa_{g} \sin \theta s, \\
a_{1}^{1} & =\sin \theta+\kappa_{g} \sin \theta r+\kappa_{g} \cos \theta s, \\
a_{2}^{1} & =\cos \theta-\kappa_{g} \sin \theta s .
\end{aligned}
$$

Remark 15.2. - In order to have a right remainder, that is at least in $\mathcal{O}\left(h^{4 / 3+\eta}\right)$, for some $\eta>0$, the constant $\tau_{1}$ should satisfy the condition $\tau_{1}>1 / 3$ and $6 \delta-\tau_{1}>4 / 3$. So surely, we will impose: $1 / 3<\tau_{1}<2 / 3$. The constant $\delta$ should also satisfy: $1 / 3>\delta>1 / 6$ and will be later chosen to be quite near $1 / 3$.

We will for example take:

$$
\tau_{1}=3 \delta-\frac{1}{2}
$$

under the condition that

$$
\delta>\frac{5}{18}
$$

Proof. - Let us write

$$
\begin{aligned}
& \left|h D_{r} u-A_{1} u\right|^{2}+\alpha\left|h D_{s} u-A_{2} u\right|^{2} \\
& =b^{2} t^{2}\left(a_{1}^{2}+\alpha a_{2}^{2}\right)|u|^{2}+h^{2}\left|D_{r} u\right|^{2}+\alpha\left|h D_{s} u+\frac{b}{2} \kappa_{n, B} r^{2} u\right|^{2} \\
& \quad-2 b t \operatorname{Re}\left(\left[a_{1} h D_{r} u+\alpha a_{2}\left(h D_{s} u+\frac{b}{2} \kappa_{n, B} r^{2} u\right)\right] \bar{u}\right) .
\end{aligned}
$$

Using (15.3), we verify that:

$$
\left|1-\left(a_{1}^{2}+\alpha a_{2}^{2}\right)\right| \leqslant C\left[\left(r^{2}+s^{2}\right)+\left(r^{2}+s^{2}\right)^{3 / 2}\right],
$$

and this leads to

$$
\left|1-\left(a_{1}^{2}+\alpha a_{2}^{2}\right)\right| \leqslant C h^{2 \delta},
$$

on the support of $u$. 
As a consequence, we obtain:

$$
\begin{aligned}
& \left|h D_{r} u-A_{1} u\right|^{2}+\alpha\left|h D_{s} u-A_{2} u\right|^{2} \\
& =b^{2} t^{2}|u|^{2}+h^{2}\left|D_{r} u\right|^{2}+\alpha\left|h D_{s} u+\frac{b}{2} \kappa_{n, B} r^{2} u\right|^{2} \\
& \quad-2 b t \operatorname{Re}\left(\left[a_{1} h D_{r} u+\alpha a_{2}\left(h D_{s} u+\frac{b}{2} \kappa_{n, B} r^{2} u\right)\right] \bar{u}\right)-C h^{2 \delta}|t u|^{2} .
\end{aligned}
$$

Similarly to the decomposition of (15.10), we observe that:

$$
\begin{aligned}
\mid b t u & -\left.\left[a_{1} h D_{r} u+\alpha a_{2}\left(h D_{s} u+\frac{b}{2} \kappa_{n, B} r^{2} u\right)\right]\right|^{2} \\
& +\alpha\left|a_{2} h D_{r} u-a_{1}\left(h D_{s} u+\frac{b}{2} \kappa_{n, B} r^{2} u\right)\right|^{2} \\
= & b^{2} t^{2}|u|^{2}-2 b t \operatorname{Re}\left(\left[a_{1} h D_{r} u+\alpha a_{2}\left(h D_{s} u+\frac{b}{2} \kappa_{n, B} r^{2} u\right)\right] \bar{u}\right) \\
& +\left|a_{1} h D_{r} u+\alpha a_{2}\left(h D_{s} u+\frac{b}{2} \kappa_{n, B} r^{2} u\right)\right|^{2} \\
& +\alpha\left|a_{2} h D_{r} u-a_{1}\left(h D_{s} u+\frac{b}{2} \kappa_{n, B} r^{2} u\right)\right|^{2} .
\end{aligned}
$$

Using again (15.3), we get

$$
\begin{aligned}
& b^{2} t^{2}|u|^{2}-2 b t \operatorname{Re}\left(\left[a_{1} h D_{r} u+\alpha a_{2}\left(h D_{s} u+\frac{b}{2} \kappa_{n, B} r^{2} u\right)\right] \bar{u}\right) \\
& +\left|a_{1} h D_{r} u+\alpha a_{2}\left(h D_{s} u+\frac{b}{2} \kappa_{n, B} r^{2} u\right)\right|^{2} \\
& +\alpha\left|a_{2} h D_{r} u-a_{1}\left(h D_{s} u+\frac{b}{2} \kappa_{n, B} r^{2} u\right)\right|^{2} \\
& \leqslant b^{2} t^{2}|u|^{2}-2 b t \operatorname{Re}\left(\left[a_{1} h D_{r} u+\alpha a_{2}\left(h D_{s} u+\frac{b}{2} \kappa_{n, B} r^{2} u\right)\right] \bar{u}\right) \\
& +\left|h D_{r} u\right|^{2}+\alpha\left|h D_{s} u+\frac{b}{2} \kappa_{n, B} r^{2} u\right|^{2} \\
& +C h^{2 \delta}\left[\left|h D_{r} u\right|^{2}+\left|h D_{s} u+\frac{b}{2} \kappa_{n, B} r^{2} u\right|^{2}\right] .
\end{aligned}
$$

Combining the previous inequalities, we get, for any function $u \in \mathcal{D}_{0}$,

$$
\begin{aligned}
& \left|h D_{r} u-A_{1} u\right|^{2}+\alpha\left|h D_{s} u-A_{2} u\right|^{2} \\
& \geqslant\left|b t u-\left[a_{1} h D_{r} u+\alpha a_{2}\left(h D_{s} u+\frac{b}{2} \kappa_{n, B} r^{2} u\right)\right]\right|^{2} \\
& \quad+\alpha\left|a_{2} h D_{r} u-a_{1}\left(h D_{s} u+\frac{b}{2} \kappa_{n, B} r^{2} u\right)\right|^{2} \\
& \quad-C h^{2 \delta}|t u|^{2}-C h^{2 \delta}\left[\left|h D_{r} u\right|^{2}+\left|h D_{s} u+\frac{b}{2} \kappa_{n, B} r^{2} u\right|^{2}\right] .
\end{aligned}
$$


Writing that

$$
\left|h D_{r} u\right| \leqslant\left|h D_{r} u-A_{1} u\right|+C|t u|
$$

and

$$
\left|h D_{s} u+\frac{b}{2} \kappa_{n, B} r^{2} u\right| \leqslant\left|h D_{s} u-A_{2} u\right|+C|t u|,
$$

we get easily from (15.10)-(15.16) that for any $u \in \mathcal{D}_{0}$,

$$
\begin{aligned}
(1+ & \left.C h^{2 \delta}\right)\left[\left|h D_{r} u-A_{1} u\right|^{2}+\alpha\left|h D_{s} u-A_{2} u\right|^{2}\right] \\
\geqslant & \left|b t u-\left[a_{1} h D_{r} u+\alpha a_{2}\left(h D_{s} u+\frac{b}{2} \kappa_{n, B} r^{2} u\right)\right]\right|^{2} \\
& +\alpha\left|-a_{2} h D_{r} u+a_{1}\left(h D_{s} u+\frac{b}{2} \kappa_{n, B} r^{2} u\right)\right|^{2}-C h^{2 \delta}|t u|^{2} \\
= & \left|b t u-\left[a_{1} h D_{r} u+\alpha a_{2}\left(h D_{s} u+\frac{b}{2} \kappa_{n, B} r^{2} u\right)\right]\right|^{2} \\
& +\left|-\alpha^{1 / 2} a_{2} h D_{r} u+\alpha^{1 / 2} a_{1}\left(h D_{s} u+\frac{b}{2} \kappa_{n, B} r^{2} u\right)\right|^{2}-C h^{2 \delta}|t u|^{2} .
\end{aligned}
$$

We now observe that:

$$
\left|\alpha a_{2}-a_{2}^{0}\right|+\left|\alpha^{1 / 2} a_{2}+a_{2}^{1}\right|+\left|\alpha^{1 / 2} a_{1}-a_{1}^{1}\right| \leqslant C\left(r^{2}+s^{2}\right)
$$

and

$$
\left|\alpha^{1 / 2} a_{1}-\sin \theta\right|+\left|\alpha a_{2}+\cos \theta\right| \leqslant C\left(r^{2}+s^{2}\right)^{1 / 2} .
$$

Implementing these inequalities in (15.17), we get easily (15.5) from (15.17)-(15.19).

More precisely the estimate (15.18) permits indeed the comparison of the coefficients of $D_{r}$ and $D_{s}$ and the estimate (15.19) permits the treatment of the coefficients of $\kappa_{n, B} r^{2}$. Let us show how the parameter $\tau_{1}$ appears in the conclusion. As a typical error (corresponding to mixed terms), we get:

$$
\mathcal{O}\left(r^{2}\left(r^{2}+s^{2}\right)^{1 / 2}\right)|u|\left(|t u|+\left|h D_{r} u\right|+\left|h D_{s} u\right|\right)=\mathcal{O}\left(h^{3 \delta}\right)|u|\left(|t u|+\left|h D_{r} u\right|+\left|h D_{s} u\right|\right) .
$$

This can be controlled by:

$$
h^{6 \delta-\tau_{1}}|u|^{2}+h^{\tau_{1}}\left(|t u|^{2}+\left|h D_{r} u\right|^{2}+\left|h D_{s} u\right|^{2}\right),
$$

and using (15.4) (or a similar estimate with $q_{m, 0}^{h}$ ), we arrive to the right conclusion.

This ends the proof of the lemma.

Remark 15.3. - Of course, we will also have to add the previous remainders coming from the other comparison lemmas.

\subsection{Some "linearizing" change of variable}

We are now looking for a change of variables permitting to "eliminate" the slightly varying coefficient of $D_{r}$ or $D_{s}$ in (15.7). We introduce:

$$
\hat{\kappa}:=\kappa_{g}=\kappa_{g}\left(x_{0}\right) .
$$


Let us make the change of variables

$$
\begin{aligned}
& (r, s)=\Phi_{\hat{\kappa}}(p, q), \\
& r=\sin \theta p+\cos \theta q-\frac{\hat{\kappa}}{2}[-\cos \theta p+\sin \theta q]^{2}, \\
& s=-\cos \theta p+\sin \theta q-\frac{\hat{\kappa}}{2}\left[\sin (2 \theta)\left(p^{2}-q^{2}\right)+2 \cos (2 \theta) p q\right] .
\end{aligned}
$$

The map $\Phi_{\hat{\kappa}}$ is a perturbation of a rotation and, by the local inversion Theorem, is easily seen as a local diffeomorphism sending a fixed neighborhood of $(0,0)$ onto another neighborhood of $(0,0)$.

Then, for $h$ small enough, $Q^{h}$ becomes by $\Phi_{\hat{\kappa}}^{-1}$ a set $Q_{0}^{h}$ satisfying:

$$
\left.Q_{0}^{h}=\Phi_{\hat{\kappa}}^{-1}\left(Q^{h}\right) \subset\right]-C h^{\delta}, C h^{\delta}[\times]-C h^{\delta}, C h^{\delta}[.
$$

If we write

$$
D_{p}=c_{11} D_{r}+c_{12} D_{s}, \quad D_{q}=c_{21} D_{r}+c_{22} D_{s},
$$

we have:

$$
\begin{aligned}
& c_{11}=\frac{\partial r}{\partial p}=\sin \theta+\hat{\kappa} \cos \theta(-\cos \theta p+\sin \theta q) ; \\
& c_{12}=\frac{\partial s}{\partial p}=-\cos \theta-\hat{\kappa}(\sin (2 \theta) p+\cos (2 \theta) q) ; \\
& c_{21}=\frac{\partial r}{\partial q}=\cos \theta-\hat{\kappa} \sin \theta(-\cos \theta p+\sin \theta q) ; \\
& c_{22}=\frac{\partial s}{\partial q}=\sin \theta-\hat{\kappa}(-\sin (2 \theta) q+\cos (2 \theta) p) .
\end{aligned}
$$

Using again (15.21), we get

$$
\begin{aligned}
& c_{11}=\sin \theta+\hat{\kappa} \cos \theta s+\mathcal{O}\left(r^{2}+s^{2}\right) ; \\
& c_{12}=-\cos \theta-\hat{\kappa}(\cos \theta r-\sin \theta s)+\mathcal{O}\left(r^{2}+s^{2}\right) ; \\
& c_{21}=\cos \theta-\hat{\kappa} \sin \theta s+\mathcal{O}\left(r^{2}+s^{2}\right) ; \\
& c_{22}=\sin \theta+\hat{\kappa}(\sin \theta r+\cos \theta s)+\mathcal{O}\left(r^{2}+s^{2}\right) .
\end{aligned}
$$

Let us now control the measure in the change of variable. By an easy computation, we get:

$$
d r d s=\alpha_{1} d p d q \quad \text { with }\left|\alpha_{1}-1-\hat{\kappa} r\right| \leqslant C\left(r^{2}+s^{2}\right) .
$$

Similarly to Lemma 15.1 , we also get the following one.

LEMMA 15.4. - For any $\tau_{1}>0$, there exists a constant $C>0$, such that, for all $u \in \mathcal{D}_{0}$,

$$
\left(1+C h^{2 \delta}\right) q_{m, 0}^{h}(u) \geqslant\left(1-C h^{\tau_{1}}\right) q_{m, 1}^{h}(u)-C\left[\left(h^{2 \delta}+h^{\tau_{1}}\right)\|t u\|^{2}+h^{6 \delta-\tau_{1}}\|u\|^{2}\right]
$$

with

$$
q_{m, 1}^{h}(u)=\int_{\Omega_{0}^{h}}\left[h^{2}\left|D_{t} u\right|^{2}+\left|b t u-M_{0}^{h} u\right|^{2}+\left|M_{1}^{h} u\right|^{2}\right] \alpha_{1} d p d q d t
$$




$$
\left.\Omega_{0}^{h}=Q_{0}^{h} \times\right] 0, h^{\delta}[,
$$

and

$$
\begin{aligned}
& M_{0}^{h}=h D_{p}-\frac{b}{2} \cos \theta \kappa_{n, B}(\sin \theta p+\cos \theta q)^{2}, \\
& M_{1}^{h}=h D_{q}+\frac{b}{2} \sin \theta \kappa_{n, B}(\sin \theta p+\cos \theta q)^{2} .
\end{aligned}
$$

By a unitary transformation, and after control of a commutator, we get

$$
\begin{aligned}
& \left(1+C h^{1 / 2}\right) q_{m, 1}^{h}(u)+C h^{3 / 2}\|u\|^{2} \\
& \quad \geqslant q_{m, 2}^{h}(v)=\int_{\Omega_{0}^{h}}\left[h^{2}\left|D_{t} v\right|^{2}+\left|b t v-M_{0}^{h} v\right|^{2}+\left|M_{1}^{h} v\right|^{2}\right] d p d q d t
\end{aligned}
$$

for all $u$ in $\mathcal{D}_{0}$, with $v$ associated to $u$ by $v=\alpha_{1}^{1 / 2} u$.

Let us consider the new model. We first observe that the result is independent of $\hat{\kappa}$ considered as an independent parameter. The proof is moreover uniform with respect to this parameter. As a consequence, if $\Phi=\Phi_{\hat{\kappa}}$ was the transformation used above, $\Phi_{0}^{-1}$, more explicitely the transformation $(p, q) \mapsto(\tilde{r}=\sin \theta p+\cos \theta q, \tilde{s}=-\cos \theta p+\sin \theta q)$ will bring us (in the new variables $(\tilde{r}, \tilde{s}, t))$ to the initial model with $\hat{\kappa}$ replaced by 0 . This can also be done by explicit computation.

\subsection{End of the proof relative to the second zone}

So, for finding a lower bound for $q_{m, 2}^{h}(v)$ in (15.29), we have only to look at the corresponding model which was exactly the model analyzed in Section 13. Implementing inequality (13.20) and (13.17), we get

LEMMA 15.5. - There exists $\left.\delta_{1} \in\right] \frac{5}{18}, \frac{1}{3}[$ such that, for any $\delta \in] \delta_{1}, \frac{1}{3}[$, there exist constants $C>0, h_{0}>0$ and $\eta>0$ such that, for any $u \in \mathcal{D}_{0}$ and any $\left.\left.h \in\right] 0, h_{0}\right]$,

$$
\begin{aligned}
{[1+} & \left.C\left(h^{2 \delta}+h^{3 \delta-\frac{1}{2}}\right)\right] q_{m}^{h}(u)+C\left(h^{2 \delta}+h^{\frac{1}{3}+\eta}\right)\|t u\|^{2} \\
& +C\left(h^{\frac{4}{3}+\eta}+h^{3 \delta+\frac{1}{2}}+h^{\delta+\frac{13}{12}}\right)\|u\|^{2} \\
\geqslant & {\left[h b \Theta_{0}+h^{4 / 3} b^{2 / 3} \hat{\nu}_{0} \kappa_{n, B}^{2 / 3}\left(\delta_{0} \sin ^{2} \theta+\cos ^{2} \theta\right)^{2 / 3} \frac{\left(\mu^{\prime \prime}\left(\xi_{0}\right)\right)^{1 / 3}}{2}\right]\|u\|^{2}, }
\end{aligned}
$$

with $\Theta_{0}, \xi_{0}, \mu(\xi)$ defined in (2.6) and $\hat{\nu}_{0}$ defined in (2.15).

Then, using a modified version of Lemma 10.1, where $q_{\widetilde{A}^{(2)}}^{h}(u)$ and $q_{A}^{h}(u)$ are interchanged, and also Lemma 10.2 (with $q_{A 00}^{h}(u)$ and $q_{A}^{h}(u)$ interchanged), we get easily the following lemma

Lemma 15.6. - Let $\delta \in] \frac{1}{4}, \frac{1}{3}\left[, C_{0}>0\right.$. Then there exists $\tilde{C}_{0}$ such that, for any $x_{0} \in \Gamma_{H}$, for any $u \in H^{1}(\Omega)$ such that

$$
\operatorname{supp}(u) \subset\left\{x \in \bar{\Omega} ;\left|x-x_{0}\right| \leqslant C_{0} h^{\delta}\right\},
$$

for any $h \in] 0,1]$, we have:

$$
\begin{aligned}
q_{A}^{h}(u) \geqslant & {\left[h b \Theta_{0}+h^{4 / 3} \frac{\hat{\nu}_{0}}{2}\left[\mu^{\prime \prime}\left(\xi_{0}\right) \kappa_{n, B}^{2}\left(x_{0}\right)\right]^{1 / 3}\right]\|u\|^{2} } \\
& -\tilde{C}_{0}\left\|t^{1 / 2}\left(h D_{x}-A\right) u\right\|^{2}-\tilde{C}_{0}\left[q_{A}^{h}(u)\right]^{1 / 2} \cdot\left\|\left(h^{3 \delta}+h+h^{2 \delta} t+t^{2}\right) u\right\| \\
& -\tilde{C}_{0}\left\|\left(h^{\delta+\frac{1}{2}}+h^{3 \delta}+h+h^{2 \delta} t+t^{2}\right) u\right\|^{2}-\tilde{C}_{0} h^{\frac{1}{3}+\eta}\|t u\|^{2}-\tilde{C}_{0} h^{\frac{4}{3}+\eta}\|u\|^{2} .
\end{aligned}
$$


This lemma and (7.1) prove that for the ground state eigenfunction defined in (14.2), we have, for any $\varepsilon>0$,

$$
\begin{aligned}
q_{A}^{h}\left(\chi_{\gamma, \tau(h)} u^{h}\right) \geqslant & {\left[h b \Theta_{0}+h^{4 / 3} \hat{\gamma}_{0} b^{2 / 3}\right]\left\|\chi_{\gamma, \tau(h)} u^{h}\right\|^{2}-C\left(h^{1+\varepsilon}+h^{6 \delta-\varepsilon}\right)\left\|\chi_{\gamma, \tau(h)} u^{h}\right\|^{2} } \\
& -C\left\|t^{1 / 2}(h D-A) \chi_{\gamma, \tau(h)} u^{h}\right\|^{2}-C h^{-\varepsilon}\left\|t^{2} \chi_{\gamma, \tau(h)} u^{h}\right\|^{2} \\
& -C h^{\frac{1}{3}+\eta}\left\|t \chi_{\gamma, \tau(h)} u^{h}\right\|^{2}-C h^{\frac{4}{3}+\eta}\left\|\chi_{\gamma, \tau(h)} u^{h}\right\|^{2} .
\end{aligned}
$$

So, choosing $\varepsilon=3 \delta-\frac{1}{2}$, we have proved that there exists $C>O$, such that, for any $\gamma \in \Gamma_{\tau(h)}^{11}$ defined by (14.3), we have

$$
\begin{aligned}
q_{A}^{h}\left(\chi_{\gamma, \tau(h)} u^{h}\right) \geqslant & {\left[h b \Theta_{0}+h^{4 / 3} \hat{\gamma}_{0} b^{2 / 3}-C h^{17 / 12}\right]\left\|\chi_{\gamma, \tau(h)} u^{h}\right\|^{2} } \\
& -C h^{3 \delta+\frac{1}{2}}\left\|\chi_{\gamma, \tau(h)} u^{h}\right\|^{2}-C\left\|t^{1 / 2}(h D-A) \chi_{\gamma, \tau(h)} u^{h}\right\|^{2} \\
& -C h^{\frac{1}{2}-3 \delta}\left\|t^{2} \chi_{\gamma, \tau(h)} u^{h}\right\|^{2} .
\end{aligned}
$$

Summing over $\gamma \in \Gamma_{\tau(h)}^{11}$, we will obtain

LEMma 15.7. - There exists $\left.\delta_{1} \in\right] \frac{5}{18}, \frac{1}{3}[$, such that, for any $\delta \in] \delta_{1}, \frac{1}{3}[$, there exist $C, \eta>0$ and $h_{0}$ such that, for all $\left.\left.h \in\right] 0, h_{0}\right]$,

$$
\begin{aligned}
\sum_{\gamma \in \Gamma_{\tau(h)}^{11}} q_{A}^{h}\left(\chi_{\gamma, \tau(h)} u^{h}\right) \geqslant & {\left[h b \Theta_{0}+h^{4 / 3} \hat{\gamma}_{0} b^{2 / 3}\right] \sum_{\gamma \in \Gamma_{\tau(h)}^{11}}\left\|\chi_{\gamma, \tau(h)} u^{h}\right\|^{2} } \\
& -C h^{3 \delta+\frac{1}{2}}-C h^{\frac{4}{3}+\eta} .
\end{aligned}
$$

Proof. - Let

$$
\begin{aligned}
r_{\gamma}^{11}(h):= & C\left(h^{3 \delta+\frac{1}{2}}+h^{\frac{4}{3}+\eta}\right)\left\|\chi_{\gamma, \tau(h)} u^{h}\right\|^{2}+C\left\|t^{1 / 2}(h D-A) \chi_{\gamma, \tau(h)} u^{h}\right\|^{2} \\
& +C h^{\frac{1}{2}-3 \delta}\left\|t^{2} \chi_{\gamma, \tau(h)} u^{h}\right\|^{2}+C h^{\frac{1}{3}+\eta}\left\|\chi_{\gamma, \tau(h)} t u^{h}\right\|^{2} .
\end{aligned}
$$

We obtain:

$$
\begin{aligned}
\sum_{\gamma \in \Gamma_{\tau(h)}^{11}} r_{\gamma}^{11}(h) \leqslant & C h^{3 \delta+\frac{1}{2}} \sum_{\gamma \in \Gamma_{\tau(h)}^{11}}\left\|\chi_{\gamma, \tau(h)} u^{h}\right\|^{2}+C h^{\frac{1}{2}-3 \delta} \sum_{\gamma \in \Gamma_{\tau(h)}^{11}}\left\|t^{2} \chi_{\gamma, \tau(h)} u^{h}\right\|^{2} \\
& +C \sum_{\gamma \in \Gamma_{\tau(h)}^{11}}\left\|t^{1 / 2}(h D-A) \chi_{\gamma, \tau(h)} u^{h}\right\|^{2} .
\end{aligned}
$$

We use then Proposition 7.1 for the first two terms of the right hand side. The last term is treated in the following way. As for (7.15), we have also

$$
\left\|t^{1 / 2}(h D-A) u^{h}\right\|^{2}=\sum_{\gamma}\left[\left\|t^{1 / 2}(h D-A) \chi_{\gamma, \tau(h)} u^{h}\right\|^{2}-h^{2}\left\|t^{1 / 2}\left|\nabla \chi_{\gamma, \tau(h)}\right| u^{h}\right\|^{2}\right] .
$$

So

$$
\sum_{\gamma \in \Gamma_{\tau(h)}^{11}}\left\|t^{1 / 2}(h D-A) \chi_{\gamma, \tau(h)} u^{h}\right\|^{2} \leqslant C\left(h^{3 / 2}+h^{3-2 \delta}\right) .
$$




\section{Lower bounds in the fourth zone and end of the proof of Theorem 1.2}

\subsection{Introduction}

As for the second zone, we assume that: $\operatorname{supp}\left(\chi_{\gamma, \tau(h)}\right) \subset Q\left(x_{0}\right)$, but with $x_{0}=\left(r_{0}, 0, s_{0}\right)$, $r_{0} \neq 0$. For simplicity of notation, we take $s_{0}=0$. We have also to consider the case when $J=\left[-C h^{\delta}, C h^{\delta}\right]$ and $I=r_{0}+\left[-h^{\delta}, h^{\delta}\right]$, with $\left|r_{0}\right|$ small enough. We will also impose $\left|r_{0}\right| \geqslant C_{1} h^{\delta}$, with $C_{1}$ large enough in order to have the property that the corresponding $I(h)$ is always sufficiently far from 0 .

\subsection{Comparison}

One first observes that the proof of the rough lower bound will permit to treat the region $r \geqslant h^{\delta_{1}}$ (but the conditions on $\delta_{1}$ are too bad for what we need). We proceed as in the proof of Proposition 9.2 of [12] and use the partition of unity introduced in Section 7. We follow the first steps of Section 7.3 till (7.25). We recall that there exist local coordinates ${ }^{7}$ adapted to the boundary $y=\left(y_{1}, y_{2}, y_{3}\right)$ such that $y_{3}(x)=d(x, \partial \Omega)$ and that, for $\tau(h)<1$ and for any $k \in \mathbb{N}^{\star}$,

$$
\begin{aligned}
q_{A}^{h}\left(\chi_{\gamma, \tau(h)} u\right) \geqslant & (1-C \tau(h)) q_{\widetilde{A}^{(k)}}^{h}\left(\chi_{\gamma, \tau(h)} u\right) \\
& -C(\tau(h))^{k+1}\left\|\chi_{\gamma, \tau(h)} u\right\| \cdot\left(q_{A}^{h}\left(\chi_{\gamma, \tau(h)} u\right)\right)^{1 / 2} \\
& -C(\tau(h))^{2 k+2}\left\|\chi_{\gamma, \tau(h)} u\right\|^{2}
\end{aligned}
$$

with, for some $y_{0} \in \partial \Omega \cap\left\{\tau(h)+\operatorname{supp}\left(\chi_{\gamma, \tau(h)}\right)\right\}, \widetilde{A}^{(k)}$ and $q_{\widetilde{A}^{(k)}}^{h}$ defined in (7.20) and (7.21). If we take $\tau(h)=h^{\delta}$ and $k=1$ the estimate we got in Section 7 is good when

$$
d\left(y_{0}, \Gamma_{H}\right) \geqslant C_{0} h^{2 \delta-1 / 2}
$$

with $C_{0}$ large enough. So we have to take $k=2$ for the general case, and we will then consider the condition:

$$
d\left(y_{0}, \Gamma_{H}\right) \geqslant C_{1} h^{\delta}
$$

\subsection{Adapted normal forms}

We can always by a change of variable

$$
\left(y_{1}, y_{2}\right) \mapsto\left(\cos \omega y_{1}-\sin \omega y_{2}, \sin \omega y_{1}+\cos \omega y_{2}\right),
$$

with

$$
\cos \omega \widetilde{H}_{2}^{0}+\sin \omega \widetilde{H}_{1}^{0}=0,
$$

arrive to a situation where the new second component of $\widetilde{H}$ vanishes:

$$
\widetilde{H}_{2}^{0}=0 .
$$

Then, for suitable gauge,

\footnotetext{
${ }^{7}$ Belonging to the family introduced in Section 5.3.
} 


$$
\begin{aligned}
& \widetilde{A}_{1}^{(2)}(y)=\tilde{c}_{1}^{0} y_{2}^{2}+\widetilde{R}_{1,2} y_{2} y_{3}+\widetilde{R}_{1,3} y_{3}^{2}, \\
& \widetilde{A}_{2}^{(2)}(y)=-\widetilde{H}_{1}^{0} y_{3}+\widetilde{H}_{3}^{0} y_{1}+\widetilde{c}_{2}^{0} y_{1}^{2}+\widetilde{R}_{2,1} y_{1} y_{3}+\widetilde{R}_{2,3} y_{3}^{2}, \\
& \widetilde{A}_{3}^{(2)}(y)=\widetilde{R}_{3,1} y_{1} y_{3}+\widetilde{R}_{3,2} y_{2} y_{3} .
\end{aligned}
$$

Let us define $\widetilde{A}^{(2,0)}$ as $\widetilde{A}^{(2)}$ defined above but with $R_{i j}=0$ :

$$
\begin{aligned}
& \widetilde{A}_{1}^{(2,0)}(y)=\tilde{c}_{1}^{0} y_{2}^{2}, \\
& \widetilde{A}_{2}^{(2,0)}(y)=-\widetilde{H}_{1}^{0} y_{3}+\widetilde{H}_{3}^{0} y_{1}+\widetilde{c}_{2}^{0} y_{1}^{2}, \\
& \widetilde{A}_{3}^{(2,0)}(y)=0 .
\end{aligned}
$$

As for (16.1), we have

(16.4)

$$
\begin{aligned}
q_{A}^{h}\left(\chi_{\gamma, \tau(h)} u\right) \geqslant & (1-C \tau(h)) q_{\widetilde{A}^{(2,0)}}^{h}\left(\chi_{\gamma, \tau(h)} u\right) \\
& -C \tau(h)\left\|t \chi_{\gamma, \tau(h)} u\right\| \cdot\left(q_{A}^{h}\left(\chi_{\gamma, \tau(h)} u\right)\right)^{1 / 2} \\
& -C(\tau(h))^{3}\left\|\chi_{\gamma, \tau(h)} u\right\| \cdot\left(q_{A}^{h}\left(\chi_{\gamma, \tau(h)} u\right)\right)^{1 / 2} \\
& -C(\tau(h))^{2}\left\|t \chi_{\gamma, \tau(h)} u\right\|^{2} \\
& -C(\tau(h))^{6}\left\|\chi_{\gamma, \tau(h)} u\right\|^{2} .
\end{aligned}
$$

We recall that $t=y_{3}$. So

$$
\begin{aligned}
(1+C \tau(h)) q_{A}^{h}\left(\chi_{\gamma, \tau(h)} u\right) \geqslant & (1-C \tau(h)) q_{\widetilde{A}^{(2,0)}}^{h}\left(\chi_{\gamma, \tau(h)} u\right) \\
& -C \tau(h)\left\|t \chi_{\gamma, \tau(h)} u\right\|^{2} \\
& -C(\tau(h))^{5}\left\|\chi_{\gamma, \tau(h)} u\right\|^{2} .
\end{aligned}
$$

If we assume that $d\left(y_{0}, \Gamma_{H}\right) \in\left[C_{1} h^{\delta}, 1 / C_{1}[\right.$, using that in (16.2) we have:

$$
\left(\widetilde{H}_{1}^{0}\right)^{2}+\left(\widetilde{H}_{3}^{0}\right)^{2}=b^{2}
$$

and the hypothesis (1.7), then, in (16.2), we get

$$
\begin{aligned}
& \frac{d\left(y_{0}, \Gamma_{H}\right)}{C} \leqslant\left|\widetilde{H}_{3}^{0}\right| \leqslant C d\left(y_{0}, \Gamma_{H}\right), \\
& \| \widetilde{H}_{1}^{0}|-b| \leqslant C d^{2}\left(y_{0}, \Gamma_{H}\right), \\
& \left|\tilde{c}_{1}^{0}\right|+\left|\tilde{c}_{2}^{0}\right| \leqslant C .
\end{aligned}
$$

\subsection{Analysis of the model}

LEMma 16.1. - For all $\delta \in] 0, \frac{1}{3}\left[\right.$, for all $C>0$, there exist $c_{1}$ and $C_{1}^{1}$ such that if $C_{1} \geqslant C_{1}^{1}$, then, if

$$
d\left(y_{0}, \Gamma_{H}\right) \in\left[C_{1} h^{\delta}, \frac{1}{C_{1}}[\right.
$$

is satisfied,

$$
q_{\widetilde{A}^{(2,0)}}^{h}(v) \geqslant h b\left[\Theta_{0}+c_{1} d\left(y_{0}, \Gamma_{H}\right)\right]\|v\|^{2},
$$

for any function $v$ in $C_{0}^{\infty}(]-C h^{\delta}, C h^{\delta}\left[{ }^{2} \times \overline{\mathbb{R}^{+}}\right)$. 
Proof. - Making the dilation

$$
r=h^{-1 / 3} b_{0}^{1 / 2} \tilde{r}, \quad s=h^{-1 / 3} b_{0}^{1 / 2} \tilde{s}, \quad t=h^{-1 / 2} b_{0}^{-1 / 2} \tilde{t}
$$

with $b_{0}=\left|\widetilde{H}_{1}^{0}\right|$, we get that

$$
q_{\widetilde{A}^{(2,0)}}^{h}(v)=h b_{0} q_{0}(w)=h b_{0}\left[\left\|D_{t} w\right\|^{2}+\left\|\left(t-L_{1}^{h}\right) w\right\|^{2}+\left\|L_{2}^{h} w\right\|^{2}\right],
$$

with

$$
\begin{aligned}
L_{1}^{h} & =h^{1 / 6} D_{\tilde{s}}+h^{-1 / 6} d_{1} \tilde{r}+h^{1 / 6} d_{2} \tilde{r}^{2}+h^{1 / 6} d_{3} \tilde{r} \tilde{s}, \\
L_{2}^{h} & =h^{1 / 6} D_{\tilde{s}},
\end{aligned}
$$

and $w \in C_{0}^{\infty}(]-C h^{\delta-1 / 3}, C h^{\delta-1 / 3}\left[{ }^{2} \times \overline{\mathbb{R}^{+}}\right)$, related to $v$ by:

$$
w(\tilde{r}, \tilde{s}, \tilde{t})=h^{7 / 12} b_{0}^{-1 / 4} v(r, s, t) .
$$

We now omit the tilda's. Here we observe that:

$$
\left[L_{1}^{h}, L_{2}^{h}\right]=i\left(d_{1}+2 h^{1 / 3} d_{2} r+h^{1 / 3} d_{3} s\right),
$$

and

$$
\left[\left[L_{1}^{h}, L_{2}^{h}\right], L_{1}^{h}\right]=-h^{1 / 2} d_{2}
$$

But

$$
q_{0}(w) \geqslant\left\langle\mu\left(L_{1}^{h}+\xi_{0}\right) w, w\right\rangle+\left\|L_{2}^{h} w\right\|^{2} .
$$

As the function $\mu$ is of class $C^{\infty}$ on $\mathbb{R}$ and satisfies the properties recalled in Section 2.2, it is easy to find a real $C^{\infty}$ function $f$ satisfying

$$
\mu(t)-\Theta_{0} \geqslant f^{2}(t),
$$

$$
f^{\prime}(t) \geqslant 0 \text {, with strict inequality in a neighborhood of } \xi_{0} \text {, }
$$

$f$ is constant at $\pm \infty$.

The main property is here that there exists $\rho_{1}>0$ such that:

$$
f^{\prime}(t)+f(t)^{2} \geqslant \rho_{1} \text {. }
$$

Writing that

$$
q_{0}(w)-\Theta_{0}\|w\|^{2} \geqslant\left\|f\left(L_{1}^{h}+\xi_{0}\right) w\right\|^{2}+\left\|L_{2}^{h} w\right\|^{2},
$$

we use a standard bracket argument to get that

$$
q_{0}(w)-\Theta_{0}\|w\|^{2} \geqslant\left|\left\langle\left[f\left(L_{1}^{h}+\xi_{0}\right), L_{2}^{h}\right] w, w\right\rangle\right| .
$$


But this bracket can be computed as:

$$
\left[f\left(L_{1}^{h}+\xi_{0}\right), L_{2}^{h}\right]=f^{\prime}\left(L_{1}^{h}+\xi_{0}\right)\left[L_{1}^{h}, L_{2}^{h}\right]-\frac{1}{2} f^{\prime \prime}\left(L_{1}^{h}+\xi_{0}\right)\left[\left[L_{1}^{h}, L_{2}^{h}\right], L_{1}^{h}\right] .
$$

One way to get this formula is to use a gauge transformation for having:

$$
\begin{aligned}
& L_{1}^{h, \text { new }}=h^{1 / 6} D_{s}, \\
& L_{2}^{h, \text { new }}=h^{1 / 6} D_{r}-h^{-1 / 6} d_{1} s-2 h^{1 / 6} d_{2} r s-h^{1 / 6} d_{3} s^{2} .
\end{aligned}
$$

Taking the Fourier transform with respect to all variables, we are left with the commutation of a second order differential operator with a function and the proof is easy.

So, for any $w \in C_{0}^{\infty}(]-C h^{\delta-1 / 3}, C h^{\delta-1 / 3}\left[{ }^{3}\right)$

$$
\left|\left\langle\left[f\left(L_{1}^{h}+\xi_{0}\right), L_{2}^{h}\right] w, w\right\rangle\right| \geqslant\left|d_{1}\right|\left\langle\left(L_{1}^{h}+\xi_{0}\right) f^{\prime}\left(L_{1}^{h}+\xi_{0}\right) w, w\right\rangle-C h^{\delta}\|w\|^{2} .
$$

Then, by (16.11) and (16.14), we obtain:

$$
q_{0}(w) \geqslant \Theta_{0}\|w\|^{2}+C^{-1} d\left(y_{0}, \Gamma_{H}\right)\left\langle f^{\prime}\left(L_{1}^{h}+\xi_{0}\right) w, w\right\rangle-C h^{\delta}\|w\|^{2} .
$$

But, remembering that:

$$
q_{0}(w) \geqslant\left\langle\mu\left(L_{1}^{h}+\xi_{0}\right) w, w\right\rangle,
$$

the properties (16.21), (16.22) and (16.23) combined with (16.15) and (16.16), prove that, for some $c_{1}>0$ and if $C_{1} h^{\delta} \leqslant d\left(y_{0}, \Gamma_{H}\right) \leqslant C_{1}^{-1}$, then

$$
q_{0}(w) \geqslant\left(\Theta_{0}+c_{1} d\left(y_{0}, \Gamma_{H}\right)\right)\|w\|^{2} .
$$

The lemma follows.

From the estimate (16.8) of Lemma 16.1, we can recover in the fourth zone.

LEMMA 16.2. - There exist $\hat{c}_{0}>0, C_{2}$ and $C_{1}^{1}$, such that, if $C_{1}^{-1} \geqslant d\left(\tau(h) \gamma, \Gamma_{H}\right) \geqslant C_{1} h^{\delta}$, with $C_{1} \geqslant C_{1}^{1}$ then, for any $u \in H^{1}(\Omega)$,

$$
q_{A}^{h}\left(\chi_{\gamma, \tau(h)} u\right) \geqslant h\left(b \Theta_{0}+\hat{c}_{0} d\left(\tau(h) \gamma, \Gamma_{H}\right)\right)\left\|\chi_{\gamma, \tau(h)} u\right\|^{2}-C_{2} h^{\delta}\left\|t \chi_{\gamma, \tau(h)} u\right\|^{2}
$$

for all $\gamma \in \Gamma_{\tau(h)}^{12}$.

\subsection{Control of the remainders}

We have now to control the remainders appearing in the right hand side of (16.25). We plan indeed to use this estimate with $u=u^{h}$ and to sum over the $\gamma^{\prime}$ s in $\Gamma_{\tau(h)}^{12}$. More precisely, we will show:

Lemma 16.3. - Let $\delta \in] \frac{1}{4}, \frac{1}{3}\left[\right.$. There exist $\tilde{c}_{0}>0, C$ and $C_{1}^{1}$, such that, if $C_{1}$ satisfies $C_{1} \geqslant C_{1}^{1}$ then

$$
q_{A}^{h}\left(\chi_{\gamma, \tau(h)} u\right) \geqslant h\left(b \Theta_{0}+\tilde{c}_{0} C_{1} h^{\delta}\right)\left\|\chi_{\gamma, \tau(h)} u^{h}\right\|^{2}-r_{\gamma}^{12}(h),
$$


for all $\gamma \in \Gamma_{\tau(h)}^{12}$, with

$$
\sum_{\gamma \in \Gamma_{\tau(h)}^{12}} r_{\gamma}^{12} \leqslant C h^{2-2 \delta}
$$

Proof. - Using Lemma 16.2, the remaining problem is with the term $h^{\delta} \sum_{\gamma}\left\|t \chi_{\gamma} u^{h}\right\|^{2}$. Proposition 7.1 indeed gives that:

$$
h^{\delta} \sum_{\gamma}\left\|t \chi_{\gamma} u^{h}\right\|^{2} \leqslant h^{\delta}\left\|t u^{h}\right\|^{2} \leqslant C h^{1+\delta},
$$

but this error is too large. One could think that one could get instead the better:

$$
h^{\delta} \sum_{\gamma \in \Gamma_{\tau(h)}^{12}}\left\|t \chi_{\gamma} u^{h}\right\|^{2} \leqslant C h^{1+\delta} \sum_{\gamma \in \Gamma_{\tau(h)}^{12}}\left\|\chi_{\gamma} u^{h}\right\|^{2},
$$

but this is probably not true. We will actually show the weaker:

$$
h^{\delta} \sum_{\gamma \in \Gamma_{\tau(h)}^{12}}\left\|t \chi_{\gamma} u^{h}\right\|^{2} \leqslant C^{\prime} h^{1+\delta} \sum_{\gamma \in \Gamma_{\tau(h)}^{12}}\left\|\chi_{\gamma} u^{h}\right\|^{2}+\mathcal{O}\left(h^{2-\delta}\right) .
$$

In order to get this more accurate upper bound, we need a:

Local control of $\left\|t \chi u^{h}\right\|$. We follow the proof of Proposition 7.1, but we have to take account of the presence of $\chi$. One first obtains (see (7.4)) that:

$$
h b \int t^{2} \chi^{2}\left|u^{h}\right|^{2} d x \leqslant \int t^{2}\left|(h D-A) \chi u^{h}\right|^{2} d x+C h\left\|(h D-A) \chi u^{h}\right\| \cdot\left\|t \chi u^{h}\right\| .
$$

Commuting $\chi$ and $(h D-A)$ in the second term of the right hand side, we get:

$$
\begin{aligned}
h b \int t^{2} \chi^{2}\left|u^{h}\right|^{2} d x \leqslant & \int t^{2}\left|(h D-A) \chi u^{h}\right|^{2} d x+C h\left\|\chi(h D-A) u^{h}\right\| \cdot\left\|t \chi u^{h}\right\| \\
& +C h\left\|t|\nabla \chi|(h D-A) u^{h}\right\| \cdot\left\|t \chi u^{h}\right\| .
\end{aligned}
$$

Let us now analyze $\int t^{2}\left|(h D-A) \chi u^{h}\right|^{2}$. Starting from:

$$
\int t^{2}\left|(h D-A) \chi u^{h}\right|^{2} d x=\int t^{2}\left(\chi(h D-A) u^{h}+h \nabla \chi u^{h}\right) \cdot\left(\overline{\chi(h D-A) u^{h}+h \nabla \chi u^{h}}\right) d x
$$

we get:

This leads to

$$
\begin{aligned}
\int t^{2}\left|(h D-A) \chi u^{h}\right|^{2} d x \leqslant & \int t^{2} \chi^{2}(h D-A)^{2} u^{h} \overline{u^{h}} d x+h^{2} \int t^{2}|\nabla \chi|^{2}\left|u^{h}\right|^{2} d x \\
& +2 h\left\|t \chi u^{h}\right\|\left\|t(\nabla \chi) \cdot(h D-A) u^{h}\right\| \\
& +C h\left\|t \chi u^{h}\right\|\left\|\chi(h D-A) u^{h}\right\| .
\end{aligned}
$$

$$
\begin{aligned}
\int t^{2}\left|(h D-A) \chi u^{h}\right|^{2} d x \leqslant & \lambda(h) \int t^{2}\left|\chi u^{h}\right|^{2} d x+h^{2} \int t^{2}|\nabla \chi|^{2}\left|u^{h}\right|^{2} d x \\
& +2 h\left\|t \chi u^{h}\right\|\left\|t(\nabla \chi) \cdot(h D-A) u^{h}\right\| \\
& +C h\left\|t \chi u^{h}\right\|\left\|t \chi(h D-A) u^{h}\right\| .
\end{aligned}
$$


We then get:

$$
\begin{aligned}
(h b-\lambda(h)) \int t^{2}\left|\chi u^{h}\right|^{2} d x \leqslant & h^{2} \int t^{2}|\nabla \chi|^{2}\left|u^{h}\right|^{2} d x \\
& +C h\left\|t \chi u^{h}\right\|\left\|t \chi(h D-A) u^{h}\right\| \\
& +C h\left\|t|\nabla \chi|(h D-A) u^{h}\right\| \cdot\left\|t \chi u^{h}\right\| .
\end{aligned}
$$

This leads to the estimate:

$$
\left\|t \chi u^{h}\right\|^{2} \leqslant C\left[\left\|\chi(h D-A) u^{h}\right\|+\left\|t|\nabla \chi|(h D-A) u^{h}\right\|\right]\left\|t \chi u^{h}\right\|+h \int t^{2}|\nabla \chi|^{2}\left|u^{h}\right|^{2} d x
$$

and consequently

$$
\left\|t \chi u^{h}\right\|^{2} \leqslant C\left[\left\|\chi(h D-A) u^{h}\right\|^{2}+\left\|t|\nabla \chi|(h D-A) u^{h}\right\|^{2}+h \int t^{2}|\nabla \chi|^{2}\left|u^{h}\right|^{2} d x\right] .
$$

On the other hand, we have:

$$
\left\|\chi(h D-A) u^{h}\right\|^{2} \leqslant \lambda(h)\left\|\chi u^{h}\right\|^{2}+2\left\|\chi(h D-A) u^{h}\right\| \cdot\left\||\nabla \chi| u^{h}\right\|
$$

and consequently to:

$$
\left\|\chi(h D-A) u^{h}\right\|^{2} \leqslant C\left[h\left\|\chi u^{h}\right\|^{2}+h^{2}\left\||\nabla \chi| u^{h}\right\|^{2}\right] .
$$

Finally the two estimates (16.30) and (16.31) give

$$
\left\|t \chi u^{h}\right\|^{2} \leqslant C\left[h\left\|\chi u^{h}\right\|^{2}+h^{2}\left\||\nabla \chi| u^{h}\right\|^{2}+h\left\|t|\nabla \chi| u^{h}\right\|^{2}+\left\|t|\nabla \chi|(h D-A) u^{h}\right\|^{2}\right] .
$$

We now take $\chi=\chi_{\gamma}$ and sum over $\gamma \in \Gamma_{\tau(h)}^{12}$. Using Proposition 7.1, we get, with

$$
\begin{gathered}
r_{\gamma}^{1}:=h^{2}\left\|\left|\nabla \chi_{\gamma}\right| u^{h}\right\|^{2}+h\left\|t\left|\nabla \chi_{\gamma}\right| u^{h}\right\|^{2}+\left\|t\left|\nabla \chi_{\gamma}\right|(h D-A) u^{h}\right\|^{2}, \\
\sum_{\gamma \in \Gamma_{\tau(h)}^{12}} r_{\gamma}^{1} \leqslant C h^{2-2 \delta} .
\end{gathered}
$$

\subsection{End of the proof}

Then (14.1), (14.7), (14.4), (16.26), (15.34) and (7.15) prove that, for a suitable choice of $\delta$ ( $\delta=\frac{3}{10}$ is enough), there exist $\eta>0, C$ and $h_{0}$ such that, for all $\left.\left.h \in\right] 0, h_{0}\right]$,

$$
\lambda(h) \geqslant\left[h b \Theta_{0}+h^{4 / 3} \hat{\gamma}_{0} b^{2 / 3}\right]-C h^{\frac{4}{3}+\eta} .
$$

This corresponds to the announced lower bound in Theorem 1.2.

\section{Conclusion}

In this article, we have established and proved a new conjecture extending Bernoff-Sternberg conjecture in the case of dimension 3. As in [12], one can expect applications to the localization 
of the ground states which was mentioned in the introduction as our main motivation. We hope to come back to these points in a near future (see [26]). We observe also that the possible role of these lower bounds for problems in superconductivity is, in the same spirit of what was done in the case of dimension 2 in Helffer and Pan [17], developed in [26].

\section{Acknowledgements}

The first author would like to thank X-B. Pan for transmitting his preprint [27] before publication in November 2001 and his preprint [26] just after the publication of our preliminary results in mp_arc [14]. He is also grateful to the organizers of the conferences, workshops or seminars in Goa (December 2000), Cardiff (July 2001), Stockholm (August 2001), Grenoble (December 2001), Reims (February 2002), Orsay (March 2002), Nantes (June 2002) and Munich (July 2002), where some of these results were presented.

\section{REFERENCES}

[1] Agmon S., Lectures on Exponential Decay of Solutions of Second Order Elliptic Equations, in: Math. Notes, vol. 29, Princeton University Press, 1982.

[2] Bauman P., Phillips D., TANG Q., Stable nucleation for the Ginzburg-Landau system with an applied magnetic field, IMA Preprint Series 1416 (1996), Arch. Rational Mech. Anal. 142 (1998) $1-43$.

[3] Bernoff A., Sternberg P., Onset of superconductivity in decreasing fields for general domains, J. Math. Phys. 39 (1998) 1272-1284.

[4] Bolley C., Modélisation du champ de retard à la condensation d'un supraconducteur par un problème de bifurcation, $M^{2} A N 26$ (2) (1992) 235-287.

[5] E.B. DAvies, Spectral Theory and Differential Operators, in: Cambridge Studies in Advanced Mathematics.

[6] Dauge M., HelfFer B., Eigenvalues variation I, Neumann problem for Sturm-Liouville operators, J. Differential Equations 104 (2) (1993) 243-262.

[7] Dierkes U., Hildebrandt S., Kuster A., Wohlrab O., Minimal Surfaces I, Springer-Verlag, Berlin, 1992.

[8] HelfFer B., Introduction to the Semiclassical Analysis for the Schrödinger Operator and Applications, in: Springer Lecture Notes in Math., vol. 1336, 1988.

[9] HelfFer B., Semi-classical analysis for the Schrödinger operator with magnetic wells (after R. Montgomery, B. Helffer and A. Mohamed), in: Proceedings of the Conference in Minneapolis, in: IMA Volumes in Mathematics and its Applications, vol. 95, Springer-Verlag, 1997, pp. 99-114.

[10] HelfFer B., Bouteilles magnétiques et supraconductivité (d'après Helffer-Morame, Lu-Pan et Helffer-Pan), Séminaire EDP de l'École Polytechnique 2000-2001, Reprint http://www.math. polytechnique.fr.

[11] HelfFer B., Mohamed A., Semiclassical analysis for the ground state energy of a Schrödinger operator with magnetic wells, J. Funct. Anal. 138 (1) (1996) 40-81.

[12] HelfFer B., Morame A., Magnetic bottles in connection with superconductivity, J. Funct. Anal. 185 (2) (2001) 604-680.

[13] Helffer B., Morame A., Magnetic bottles in connection with superconductivity: Case of dimension 3, Proc. Indian Acad. Sci. (Math. Sci.) 112 (1) (2002).

[14] B. HelfFer, A. Morame, Magnetic bottles for the Neumann problem: Curvature effects in the case of dimension 3, Preprint mp_arc 01-362, 2001.

[15] B. HelfFer, A. Morame, Magnetic bottles for the Neumann problem: Curvature effects in the case of dimension 3, general case, Preprint mp_arc 02-145, 2002.

[16] HelfFer B., NourRigat J., Hypoellipticité maximale pour des opérateurs polynômes de champs de vecteurs, in: Progress in Mathematics, vol. 58, Birkhäuser, 1985. 
[17] HelfFER B., PAN X.-B., Upper critical field and location of surface nucleation of superconductivity, Annales de l'Institut Henri Poincaré (Analyse Non Linéaire) 20 (1) (2003) 145-181.

[18] HelfFer B., J. SJöstrand, Multiple wells in the semiclassical limit I, Comm. PDE 9 (4) (1984) 337-408.

[19] LU K., PAN X.-B., Estimates of the upper critical field for the Ginzburg-Landau equations of superconductivity, Phys. D 127 (1999) 73-104.

[20] LU K., PAN X.-B., Eigenvalue problems of Ginzburg-Landau operator in bounded domains, J. Math. Phys. 40 (6) (1999) 2647-2670.

[21] LU K., PAN X.-B., Gauge invariant eigenvalue problems on $\mathbb{R}^{2}$ and $\mathbb{R}_{+}^{2}$, Trans. Amer. Math. Soc. 352 (3) (2000) 1247-1276.

[22] LU K., PAN X.-B., Ginzburg-Landau system and surface nucleation of superconductivity, in: Proceeding of the IMS Workshop on Reaction-Diffusion systems, Chinese University of Hong-Kong, 1999.

[23] Lu K., PAN X.-B., Surface nucleation of superconductivity in 3-dimension, J. Differential Equations 168 (2) (2000) 386-452.

[24] Montgomery R., Hearing the zerolocus of a magnetic field, Comm. Math. Phys. 168 (1995) 651675.

[25] PAn X.-B., Upper critical field for superconductors with edges and corners, Calc. Var. PDE 14 (2002) 447-482.

[26] X.-B. PAN, Surface conductivity in 3 dimensions, Personal communication, October 2001, Unpublished.

[27] PAN X.-B., KWEK K.-H., Schrödinger operators with non-degenerately vanishing magnetic fields in bounded domain, Trans. Amer. Math. Soc. 354 (10) (2002) 4201-4227.

[28] Del Pino M., Felmer P.L., Sternberg P., Boundary concentration for eigenvalue problems related to the onset of superconductivity, Comm. Math. Phys. 210 (2000) 413-446.

[29] ReEd M., Simon B., Methods of modern Mathematical Physics, IV: Analysis of Operators, Academic Press, New York, 1978.

[30] Saint-James D., Sarma G., Thomas E.J., Type II Superconductivity, Pergamon, Oxford, 1969.

[31] SPIVAK M., Differential Geometry, Publish or Perish, Houston, 1975.

[32] Thaller B., The Dirac Equation, in: Texts and Monographs in Physics, Springer-Verlag, 1992.

(Manuscrit reçu le 26 juillet 2002; accepté le 23 avril 2003.)

\author{
Bernard HELFFER \\ Département de Mathématiques, \\ UMR CNRS 8628, \\ Bât. 425, \\ Université Paris-Sud, \\ F-91405 Orsay Cedex, France \\ E-mail: Bernard.Helffer@math.u-psud.fr
}

\author{
Abderemane MORAME \\ Département de Mathématiques, \\ UMR CNRS 6629, \\ Université de Nantes, \\ 2, rue de la Houssinière, \\ B.P. 92208 , \\ 44322 Nantes Cedex 3, France
}

E-mail: morame@math.univ-nantes.fr 